\title{
Buchberger-Zacharias Theory of Multivariate Ore Extensions
}

\author{
Michela Ceria \\ Dipartimento di Matematica \\ Università di Trento \\ michela.ceria@unitn.it
}

\author{
Teo Mora \\ DIMA \\ Università di Genova \\ theomora@disi.unige.it
}

January 10, 2017

\begin{abstract}
We present Buchberger Theory and Algorithm of Gröbner bases for multivariate Ore extensions of rings presented as modules over a principal ideal domain. The algorithms are based on Möller Lifting Theorem.
\end{abstract}

In her 1978 Bachelor's thesis [53] Zacharias discussed how to extend Buchberger Theory [7, 8, 10] from the case of polynomial rings over a field to that of polynomials over a Noetherian ring with suitable effectiveness conditions.

In the meantime a similar task was performed in a series of papers - Kandri-RodyKapur [20] merged the rewriting rules behind Euclidean Algorithm with Buchberger's rewriting, proposing a Buchberger Theory for polynomial rings over Euclidean domains; Pan [39] studied Buchberger Theory for polynomial rings over domains introducing the notions of strong/weak Gröbner bases - which culminated with [34].

Such unsorpassed paper, reformulating and improving Zacharias' intuition, gave efficient solutions to compute both weak and strong Gröbner bases for polynomial rings over each Zacharias ring, with particolar attention to the PIR case. Its main contribution is the reformulation of Buchberger test/completion ("a basis $F$ is Gröbner if and only if each S-polynomial between two elements of $F$, reduces to 0 ") in the more flexible lifting theorem ("a basis $F$ is Gröbner if and only if each element in a minimal basis of the syzygies among the leading monomials lifts, via Buchberger reduction, to a syzygy among the elements of $F$ "). The only further contribution to this ultimate paper is the survey [6] of Möller's results which reformulated them in terms of Szekeres Theory [50].

The suggestion of extending Buchberger Theory to non-commuative rings which satisfy Poincaré-Birkhoff-Witt Theorem was put forward by Bergman [5], effectively applied by Apel-Lassner [3, 4] to Lie algebras and further extended to solvable polynomial rings [21, 22], skew polynomial rings [15, 16, 17] and to other algebras [1, 11, 25, 26] which satisfy Poincaré-Birkhoff-Witt Theorem and thus, under the standard intepretation of Buchberger Theory in terms of filration/graduations [2, 29, 32, 49, 28], have the classical polynomial ring as associated graded rings. 
In particular Weispfenning [52] adapted his results to a generalization of the Ore extension [38] proposed by Tamari [51] and its construction was generalized by his student Pesch [40, 41] thus introducing the notion of iterative Ore extension with commuting variables

$$
\mathrm{R}:=R\left[Y_{1} ; \alpha_{1}, \delta_{1}\right]\left[Y_{2} ; \alpha_{2}, \delta_{2}\right] \cdots\left[Y_{n} ; \alpha_{n}, \delta_{n}\right], R \text { a domain; }
$$

the concept has been called Ore algebra in [12] and is renamed here as multivariate Ore extension (for a different and promising approach to Ore algebras see [23]).

Bergman's approach and most of all extensions are formulated for rings which are vector spaces over a field; in our knowledge the only instances in which the coefficient ring $R$ is presented as a $\mathbb{D}$-module over a domain $\mathbb{D}$ (or at least as a $\mathbb{Z}$-module) are Pritchard's [42, 43] extension of Möller Lifting Theory to non-commutative free algebras and Reinert's [44, 45] deep study of Buchberger Theory on Function Rings.

Following the recent survey on Buchberger-Zacharias Theory for monoid rings $R[\mathrm{~S}$ ] over a unitary effective ring $R$ and an effective monoid $\mathrm{S}$ [31], we propose here a Möller-Pritchard lifting theorem presentation of Buchberger-Zacharias Theory and related Gröbner basis computation algorithms for multivariate Ore extensions. The twist w.r.t. [31] is that there $R[\mathrm{~S}$ ] coincides with its associated graded ring; here $\mathrm{R}$ and its associated graded ring

$$
G(\mathrm{R}):=R\left[Y_{1} ; \alpha_{1}\right]\left[Y_{2} ; \alpha_{2}\right] \cdots\left[Y_{n} ; \alpha_{n}\right]
$$

coincide as sets and as left $R$-modules between themselves and with the commutative polynomial ring $R\left[Y_{1}, \cdots, Y_{n}\right]$, but as rings have different multiplications.

We begin by recalling Ore's original theory [38] of non-commutative polynomials $R[Y]$, relaxing the original assumption that $R$ is a field to the case in which $R$ is a domain (Section 1.1) and Pesch's constructions of multivariate Ore extensions (Section 1.2) and graded multivariate Ore extensions (Section 1.3), focusing on the arithmetics of the main Example 14

$$
\mathrm{R}:=R\left[Y_{1} ; \alpha_{1}\right]\left[Y_{2} ; \alpha_{2}\right] \cdots\left[Y_{n} ; \alpha_{n}\right], R:=\mathbb{Z}[x] \quad \alpha_{i}(x):=c_{i} x^{e_{i}}, c_{i} \in \mathbb{Z} \backslash\{0\}, e_{i} \in \mathbb{N} \backslash\{0\} .
$$

Next we introduce Buchberger Theory in multivariate Ore extensions recalling the notion of term-orderings (Section 2.1), definition and main properties of left, right, bilateral and restricted Gröbner bases (Section 2.2) and Buchberger Algorithm for computing canonical forms in the case in which $R$ is a field (Section 2.3).

We adapt to our setting Szekeres Theory [50] (Section 3), Zacharias canonical representation with related algorithm (Section 4) and Möller Lifting Theorem (Section 5).

The next Sections are the algorithmic core of the paper: we reformulate for multivariate Ore extensions over a Zacharias ring

- Möller's algorithm for computing the required Gebauer-Möller set (id est the minimal basis of the module of the syzygies among the leading monomials) for Buchberger test/completion of left weak bases (Section 6.1);

- his reformulation of it which requires only l.c.m. computation in $R$ for the case in which $R$ is a PIR (Section 6.2); 
- still in the case in which $R$ is a PIR, Möller's completion of a left weak basis to a left strong one (Section 6.3);

- Gebauer-Möller criteria [18] for producing a Gebauer-Möller set (Section 6.4);

- Kandri-Rody-Weispfenning completion [21] of a left weak bases for producing a bilateral one (Section 7.1);

- Weispfenning's [52] restricted completion (Section 7.2);

- as a technical tool required by Weispfenning's restricted completion, how to produce right Gebauer-Möller sets (Section 7.3).

Finally we reverse to a theoretical survey summarizing the structural theorem for the case in which $R$ is a Zacharias ring over a PID (Section 8), specializing to our setting Spear's Theorem [48, 28] (Section 9) and extending to it Lazard's Structural Theorem [24] (Section 10).

In an appendix we discuss, as far as it is possible, how to extend this theory and algorithms to the case in which $R$ is a PIR (Section A).

\section{Recalls on Ore Theory}

\subsection{Ore Extensions}

Let $R$ be a not necessarily commutative domain; Ore [38] investigated under which conditions the $R$-module $\mathrm{R}:=R[Y]$ of all the formal polynomials is made a ring under the assumption that the multiplication of polynomials shall be associative and both-sided distributive and the limitation imposed by the postulate that the degree of a product shall be equal to the sum of the degree of the factors.

It is clear that, due to the distributive property, it suffices to define the product of two monomials $b Y^{r} \cdot a Y^{s}$ or even more specifically, to define the product $Y \cdot r, r \in R$; this necessarily requires the existence of maps $\alpha, \delta: R \rightarrow R$ such that

$$
Y \cdot r=\alpha(r) Y+\delta(r) \text { for each } r \in R ;
$$

Ore calls $\alpha(r)$ the conjugate and $\delta(r)$ the derivative of $r$.

Under the required postulate clearly we have

1. for each $r \in R, \alpha(r)=0 \Longrightarrow r=0$,

so that $\alpha$ is injective.

It is moreover sufficient to consider, for each $r, r^{\prime} \in R$, the relations

$$
\begin{gathered}
\alpha\left(r+r^{\prime}\right) Y+\delta\left(r+r^{\prime}\right)=Y \cdot\left(r+r^{\prime}\right)=Y \cdot r+Y \cdot r^{\prime}=\left(\alpha(r)+\alpha\left(r^{\prime}\right)\right) Y+\delta(r)+\delta\left(r^{\prime}\right), \\
\alpha\left(r r^{\prime}\right) Y+\delta\left(r r^{\prime}\right)=Y \cdot\left(r r^{\prime}\right)=(Y \cdot r) \cdot r^{\prime}=\alpha(r) \alpha\left(r^{\prime}\right) Y+\alpha(r) \delta\left(r^{\prime}\right)+\delta(r) r^{\prime},
\end{gathered}
$$

and, if $R$ is a skew field, and $r \neq 0$,

$$
Y=(Y \cdot r) \cdot r^{-1}=\alpha(r) \alpha\left(r^{-1}\right) Y+\alpha(r) \delta\left(r^{-1}\right)+\delta(r) r^{-1},
$$

to deduce that 
2. $\alpha$ is a ring endomorphism;

3. the following conditions are equivalent:

(a) for each $d \in R \backslash\{0\}$ exists $c \in R \backslash\{0\}: Y \cdot c=d Y+\delta(c), \alpha(c)=d$;

(b) $\alpha$ is a ring automorphism;

4. $\delta$ is an $\alpha$-derivation of $R$ id est an additive map satisfying

$$
\delta\left(r r^{\prime}\right)=\alpha(r) \delta\left(r^{\prime}\right)+\delta(r) r^{\prime} \text { for each } r, r^{\prime} \in R
$$

5. if $R$ is a skew field, then each $r \in R \backslash\{0\}$ satisfies

$$
\alpha\left(r^{-1}\right)=(\alpha(r))^{-1}, \quad \delta\left(r^{-1}\right)=-(\alpha(r))^{-1} \delta(r) r^{-1} ;
$$

6. $\operatorname{Im}(\alpha) \subset R$ is a subring, which is an isomorphism copy of $R$;

7. $R_{1}:=\{r \in R: r=\alpha(r)\} \subset R$ is a ring, the invariant ring of $R$;

8. $R_{0}:=\{r \in R: \delta(r)=0\} \subset R$ is a ring, the constant ring of $R$;

9. $\{r \in R: Y \cdot r=r Y\}=R_{0} \cap R_{1}$.

10. If $R$ is a skew field, such are also $\operatorname{Im}(\alpha), R_{1}$ and $R_{0}$.

11. Denoting $Z:=\{z \in R: z r=r z$ for each $r \in R\}$ the center of $R$, we have

$$
\{r \in R: f \cdot r=r f \text { for each } f \in \mathrm{R}\}=R_{0} \cap R_{1} \cap Z .
$$

Moreover, if we consider two polynomials $f(Y), g(Y) \in \mathrm{R} \backslash\{0\}$,

$f=a Y^{m}+f_{0}, g=b Y^{n}+g_{0}, a, b \in R \backslash\{0\}, m, n \in \mathbb{N}, f_{0}, g_{0} \in \mathrm{R}, \operatorname{deg}\left(f_{0}\right)<m, \operatorname{deg}\left(g_{0}\right)<n$, we have

$$
f \cdot g=a \alpha^{m}(b) Y^{m+n}+h(Y), \operatorname{deg}(h)<m+n ;
$$

since $\alpha$ is an endomorphism, $b \neq 0 \Longrightarrow \alpha(b) \neq 0 \Longrightarrow \alpha^{m}(b) \neq 0$ and since $R$ is a domain it holds $\alpha^{m}(b) \neq 0 \neq a \Longrightarrow a \alpha^{m}(b) \neq 0 \Longrightarrow f \cdot g \neq 0$. As a consequence

12. $\mathrm{R}$ is a domain.

Definition 1. R with the ring structure described above is called an Ore extension and is denoted $R[Y ; \alpha, \delta]$.

Remark 2 (Ore). In an Ore extension $R[Y ; \alpha, \delta]$, denoting $\mathcal{S}=\langle\alpha, \delta\rangle$ the free semigroup over the alphabet $\{\alpha, \delta\}$ and, for each $d \in \mathbb{N}$ and $i \in \mathbb{N}, 0 \leq i \leq d, \mathcal{S}_{d, i}$ the set of the $\left(\begin{array}{l}d \\ i\end{array}\right)$ words in $\mathcal{S}$ of length $d$ in which occur $i$ instances of $\alpha$ and $d-i$ instances of $\delta$ in an arbitrary order, we have

$$
Y^{d} \cdot r=\sum_{i=0}^{d} \sum_{\tau \in \mathcal{S}_{d, i}} \tau(r) Y^{i}
$$


for each $d \in \mathbb{N}$; for instance

$$
\begin{aligned}
Y^{3} \cdot r & =\alpha^{3}(r) Y^{3}+\delta^{3}(r) \\
& +\left(\alpha^{2} \delta(r)+\alpha \delta \alpha(r)+\delta \alpha^{2}(r)\right) Y^{2} \\
& +\left(\alpha \delta^{2}(r)+\delta \alpha \delta(r)+\delta^{2} \alpha(r)\right) Y .
\end{aligned}
$$

In particular, for $f(Y)=\sum_{i=0}^{n} a_{i} Y^{n-i}$ and $g(Y)=\sum_{i=0}^{m} b_{i} Y^{m-i}$ in $\mathrm{R}$ we have

$$
g(Y) f(Y)=\sum_{i=0}^{n+m} c_{i} Y^{n+m-i} \text { with } c_{0}=b_{0} \alpha^{m}\left(a_{0}\right) \text { and } c_{i}=\sum_{\mathrm{a}=0}^{i} b_{\mathrm{a}} \sum_{\mathrm{b}=0}^{i-\mathrm{a}} \sum_{\tau \in \mathcal{S}_{m-\mathrm{a}, i \mathrm{a}-\mathrm{b}}} \tau\left(a_{\mathrm{b}}\right) \text {. }
$$

Remark 3 (Ore). Under the assumption that (cf. 3.) $\alpha$ is an automorphism, each polynomial $\sum_{i=1}^{n} a_{i} Y^{i} \in \mathrm{R}$ can be uniquely represented as $\sum_{i=1}^{n} Y^{i} \bar{a}_{i}$ for proper values $\bar{a}_{i} \in R$.

In fact we have $a x=x \alpha^{-1}(a)-\delta\left(\alpha^{-1}(a)\right)$ from which we can deduce inductively proper expressions

$$
a x^{n}=x^{n} \alpha^{-n}(a)+\sum_{i=1}^{n}(-1)^{i} x^{n-i} \sigma_{i n}(a) .
$$

\subsection{Multivariate Ore Extensions}

Definition 4. An iterative Ore extension is a ring (whose multiplication we denote $\star$ ) defined as

$$
\mathrm{R}:=R\left[Y_{1} ; \alpha_{1}, \delta_{1}\right]\left[Y_{2} ; \alpha_{2}, \delta_{2}\right] \cdots\left[Y_{n} ; \alpha_{n}, \delta_{n}\right]
$$

where, for each $i>1, \alpha_{i}$ is an endomorphism and $\delta_{i}$ an $\alpha_{i}$-derivation of the iterative Ore extension

$$
R_{i-1}:=R\left[Y_{1} ; \alpha_{1}, \delta_{1}\right] \cdots\left[Y_{i-1} ; \alpha_{i-1}, \delta_{i-1}\right] .
$$

A multivariate Ore extension (or: Ore algebra [12]; or: iterative Ore extension with commuting variables [40, 41]) is an iterative Ore extension which satisfies

- $\alpha_{j} \delta_{i}=\delta_{i} \alpha_{j}$, for each $i, j, i \neq j$,

- $\alpha_{i} \alpha_{j}=\alpha_{j} \alpha_{i}, \delta_{i} \delta_{j}=\delta_{j} \delta_{i}$ for $j>i$,

- $\alpha_{j}\left(Y_{i}\right)=Y_{i}, \delta_{j}\left(Y_{i}\right)=0$ for $j>i$.

Lemma 5 (Pesch). In an iterative Ore extension, for each $i<j$ it holds

$$
Y_{j} \star Y_{i}=Y_{i} Y_{j} \Longleftrightarrow \alpha_{j}\left(Y_{i}\right)=Y_{i}, \delta_{j}\left(Y_{i}\right)=0 .
$$

Proof. For each $i<j$, we have $Y_{j} \star Y_{i}=\alpha_{j}\left(Y_{i}\right) Y_{j}+\delta_{j}\left(Y_{i}\right)$.

Lemma 6 (Pesch). An iterative Ore extension is a multivariate Ore extension iff $Y_{j} \star$ $Y_{i}=Y_{i} Y_{j}$ for each $i<j$. 
Proof. In fact, using Lemma 5 for each $r \in R$, we have

$$
\begin{aligned}
Y_{j} \star Y_{i} \star r & =Y_{j} \star\left(\alpha_{i}(r) Y_{i}+\delta_{i}(r)\right) \\
& =\alpha_{j}\left(\alpha_{i}(r) Y_{i}+\delta_{i}(r)\right) Y_{j}+\delta_{j}\left(\alpha_{i}(r) Y_{i}+\delta_{i}(r)\right) \\
& =\alpha_{j} \alpha_{i}(r) Y_{i} Y_{j}+\alpha_{j} \delta_{i}(r) Y_{j}+\delta_{j}\left(\alpha_{i}(r) Y_{i}\right)+\delta_{j} \delta_{i}(r) \\
& =\alpha_{j} \alpha_{i}(r) Y_{i} Y_{j}+\alpha_{j} \delta_{i}(r) Y_{j}+\delta_{j} \alpha_{i}(r) Y_{i}+\delta_{j} \delta_{i}(r)
\end{aligned}
$$

and (by symmetry)

$$
\begin{aligned}
Y_{i} Y_{j} \star r & =Y_{i} \star\left(\alpha_{j}(r) Y_{j}+\delta_{i}(r)\right) \\
& =\alpha_{i} \alpha_{j}(r) Y_{i} Y_{j}+\delta_{i} \alpha_{j}(r) Y_{j}+\alpha_{i} \delta_{j}(r) Y_{i}+\delta_{i} \delta_{j}(r) .
\end{aligned}
$$

Thus the $R$-module structure of a multivariate Ore extension can be identified with that of the polynomial ring $R\left[Y_{1}, \ldots, Y_{n}\right]$ over its natural $R$-basis

$$
\mathcal{T}:=\left\{Y_{1}^{a_{1}} \cdots Y_{n}^{a_{n}}:\left(a_{1}, \ldots, a_{n}\right) \in \mathbb{N}^{n}\right\}, \quad \mathrm{R} \cong R[\mathcal{T}]=\operatorname{Span}_{R}\{\mathcal{T}\} .
$$

We can therefore denote $\alpha_{Y_{i}}:=\alpha_{i}, \delta_{Y_{i}}:=\delta_{i}$ for each $i$ and, iteratively,

$$
\alpha_{\tau Y_{i}}:=\alpha_{\tau} \alpha_{i}, \delta_{\tau Y_{i}}:=\delta_{\tau} \delta_{i}, \text { for each } \tau \in \mathcal{T} .
$$

Remark that a multivariate Ore extension is not an algebra; in fact, if we define, for $\tau=Y_{1}^{d_{1}} \cdots Y_{n}^{d_{n}}$ and $t=Y_{1}^{e_{1}} \cdots Y_{n}^{e_{n}}$ such that $\tau \mid t$

$$
\left(\begin{array}{l}
t \\
\tau
\end{array}\right):=\left(\begin{array}{l}
e_{1} \\
d_{1}
\end{array}\right) \ldots\left(\begin{array}{l}
e_{n} \\
d_{n}
\end{array}\right)
$$

we have

$$
t \star r=\sum_{\substack{\tau \in \mathcal{T} \\
\tau \mid t}}\left(\begin{array}{l}
t \\
\tau
\end{array}\right) \delta_{\frac{t}{\tau}} \alpha_{\tau}(r) \tau, \text { for each } t \in \mathcal{T} \text { and } r \in R .
$$

We can define, for each $t \in \mathcal{T}$, a map

$$
\theta_{t}: R \rightarrow \mathrm{R}, \quad \theta_{t}(r)=\sum_{\substack{\tau \in \mathcal{T} \\
\tau \mid t, \tau \neq t}}\left(\begin{array}{l}
t \\
\tau
\end{array}\right) \delta_{\frac{t}{\tau}} \alpha_{\tau}(r) \tau,
$$

so that $t \star r=\alpha_{t}(r) t+\theta_{t}(r)$ for each $t \in \mathcal{T}$ and each $r \in R$.

Such maps $\alpha_{t}$ and $\theta_{t}$ satisfy properties analogous of those of Ore's conjugate and derivative:

Lemma 7. With the present notation, for each $t \in \mathcal{T}$, we have

1. for each $r \in R, \alpha_{t}(r)=0 \Longrightarrow r=0$,

2. $\alpha_{t}$ is a ring endomorphism; 
3. the following conditions are equivalent:

(a) for each $d \in R \backslash\{0\}$ exists $c \in R \backslash\{0\}: Y \star c=d Y+\theta_{t}(c), \alpha_{t}(c)=d$;

(b) $\alpha_{t}$ is a ring automorphism;

4. $\theta_{t}$ is an $\alpha_{t}$-derivation of $\mathrm{R}$;

5. if $R$ is a skew field, then each $r \in R \backslash\{0\}$ satisfies

$$
\alpha_{t}\left(r^{-1}\right)=\left(\alpha_{t}(r)\right)^{-1}, \quad \theta_{t}\left(r^{-1}\right)=-\left(\alpha_{t}(r)\right)^{-1} \theta_{t}(r) r^{-1} ;
$$

6. $\operatorname{Im}\left(\alpha_{t}\right) \subset R$ is a subring, which is an isomorphism copy of $R$.

We further have

7. if each $\alpha_{i}$ is an automorphism, also each $\alpha_{t}, t \in \mathcal{T}$, is such.

\subsection{Associated graded Ore Extension}

Definition 8. A multivariate Ore extension

$$
R\left[Y_{1} ; \alpha_{1}, \delta_{1}\right]\left[Y_{2} ; \alpha_{2}, \delta_{2}\right] \cdots\left[Y_{n} ; \alpha_{n}, \delta_{n}\right]
$$

where each $\delta_{i}$ is zero, will be called a graded Ore extension (or: Ore extension with zero derivations [40, 41]) and will be denoted

$$
\mathrm{R}:=R\left[Y_{1} ; \alpha_{1}\right]\left[Y_{2} ; \alpha_{2}\right] \cdots\left[Y_{n} ; \alpha_{n}\right] .
$$

Lemma 9. In a multivariate graded Ore extension,

- since it is an Ore algebra, the as commute,

- and $t \star r=\alpha_{t}(r) t[=: \mathbf{M}(t \star r)]$ for each $t \in \mathcal{T}$ and $r \in R$.

Remark 10. Note that, since multivariate Ore extensions coincide, as left $R$-modules, with the classical polynomial rings $R\left[Y_{1}, \ldots, Y_{n}\right]$ and so have the same $R$-basis, namely $\mathcal{T}$, they can share with the polynomial rings their standard $\mathcal{T}$-valuation [50, 29, 2, 32] [30, §24.4,24.6]. This justifies the definition below.

Definition 11. Given an Ore extension $\mathrm{R}:=R\left[Y_{1} ; \alpha_{1}, \delta_{1}\right]\left[Y_{2} ; \alpha_{2}, \delta_{2}\right] \cdots\left[Y_{n} ; \alpha_{n}, \delta_{n}\right]$ the corresponding graded Ore extension $G(\mathrm{R}):=R\left[Y_{1} ; \alpha_{1}\right]\left[Y_{2} ; \alpha_{2}\right] \cdots\left[Y_{n} ; \alpha_{n}\right]$ is called its associated graded Ore extension.

Example 12.

1. The first non obvious example of Ore extension was proposed in 1948 by D.Tamari [51] in connection with the notion of "order of irregularity" introduced by Ore in [37]; it consists of the graded Ore extension.

$$
\mathrm{R}:=R[Y ; \alpha], R=\mathbb{Q}[x] \text { where } \alpha: R \rightarrow R: x \mapsto x^{2} .
$$


2. Such construction was generalized by Weispfenning [52] which introduced the rings

$$
\mathrm{R}:=R[Y ; \alpha], R=\mathbb{Q}[x] \text { where } \alpha: R \rightarrow R: x \mapsto x^{e}, e_{i} \in \mathbb{N}
$$

3. and extended by Pesch [40] to his iterated Ore extensions with power substitution

$$
\mathrm{R}:=R\left[Y_{1} ; \alpha_{1}\right]\left[Y_{2} ; \alpha_{2}\right] \cdots\left[Y_{n} ; \alpha_{n}\right], R=\mathbb{Q}[x]
$$

where $\alpha_{i}: R \rightarrow R: x \mapsto x^{e_{i}}, e_{i} \in \mathbb{N}$.

4. An Ore extension where $\alpha$ is invertible is discussed in [27]:

$$
\mathrm{R}:=R[S ; \alpha], R=\mathbb{Q}\left[D_{1}, D_{2}, D_{3}\right]
$$

where

$$
\alpha: R \rightarrow R: f\left(D_{1}, D_{2}, D_{3}\right) \mapsto f\left(D_{2}+2 D_{1}, D_{3},-D_{1}\right)
$$

whose inverse is

$$
\alpha^{-1}: R \rightarrow R: f\left(D_{1}, D_{2}, D_{3}\right) \mapsto f\left(-D_{3}, D_{1}+2 D_{3}, D_{2}\right) .
$$

Note that, while as $R$-modules $\mathrm{R}$ and $G(\mathrm{R})$ coincide both with the polynomial ring $\mathcal{P}=R\left[Y_{1}, \ldots, Y_{n}\right]$, the three rings have, in general, different arithmetics; we will denote $\star$ the multiplication of $\mathrm{R}$ and $*$ those of $G(\mathrm{R})$.

Example 13. The ring of Example 12, 1.

$$
\mathrm{R}:=R[Y ; \alpha], R=\mathbb{Q}[x] \text { where } \alpha: R \rightarrow R: x \mapsto x^{2}
$$

is an Ore extension which is graded.

The map

$$
\delta: \mathbb{Q}[x] \rightarrow \mathbb{Q}[x]: x^{i} \mapsto \sum_{h=i}^{2 i-1} x^{h}
$$

is an $\alpha$-derivation since

$$
\begin{aligned}
\delta\left(x^{i} \cdot x^{j}\right) & =\sum_{h=i+j}^{2 i+2 j-1} x^{h} \\
& =\sum_{h=2 i+j}^{2 i+2 j-1} x^{h}+\sum_{h=i+j}^{2 i+j-1} x^{h} \\
& =x^{2 i} \sum_{h=j}^{2 j-1} x^{h}+x^{j} \sum_{h=i}^{2 i-1} x^{h} \\
& =\alpha\left(x^{i}\right) \delta\left(x^{j}\right)+\delta\left(x^{i}\right) x^{j} ;
\end{aligned}
$$

thus $\mathrm{S}:=R[Y ; \alpha, \delta]$ is an Ore extension of which $\mathrm{R}$ is the associated graded Ore extension. 
Example 14. Since in Buchberger-Zacharias Theory, from an algorithmic point of view, the interest points are associated graded rings and thus the rôle of derivates is irrelevant, we illustrate the results for the Ore extensions with the zero-derivatives

$$
\mathrm{R}:=R\left[Y_{1} ; \alpha_{1}\right]\left[Y_{2} ; \alpha_{2}\right] \cdots\left[Y_{n} ; \alpha_{n}\right], R=\mathbb{Z}[x],
$$

with $\alpha_{i}(x):=c_{i} x^{e_{i}}, c_{i} \in \mathbb{Z} \backslash\{0\}, e_{i} \in \mathbb{N} \backslash\{0\}$.

If we denote $\gamma$ the map

$$
\gamma: \mathbb{N} \times \mathbb{N} \backslash\{0\} \rightarrow \mathbb{N},(a, e) \mapsto \sum_{i=0}^{a-1} e^{i}=\frac{1-e^{a}}{1-e}
$$

where the last equality holds for $e \neq 1$, we have $Y_{i}^{a} * x^{b}=c_{i}^{b \gamma\left(a, e_{i}\right)} x^{e_{i}^{a} b} Y_{i}^{a}$.

Note that

$$
\gamma(b, e)+e^{b} \gamma(a, e)=\sum_{i=0}^{b-1} e^{i}+\sum_{i=0}^{a-1} e^{b+i}=\sum_{i=0}^{a+b-1} e^{i}=\gamma(a+b, e)
$$

Since $\alpha_{j}\left(\alpha_{i}(x)\right)=c_{i} \alpha_{j}\left(x^{e_{i}}\right)=c_{i} c_{j}^{e_{i}} x^{e_{i} e_{j}}$ and $\alpha_{i}\left(\alpha_{j}(x)\right)=c_{j} \alpha_{i}\left(x^{e_{j}}\right)=c_{j} c_{i}^{e_{j}} x^{e_{i} e_{j}}$, then $\mathrm{R}$ is a graded Ore extension if and only if

$$
c_{i} c_{j}^{e_{i}} x^{e_{i} e_{j}}=\alpha_{j}\left(\alpha_{i}(x)\right)=\alpha_{i}\left(\alpha_{j}(x)\right)=c_{j} \alpha_{i}\left(x^{e_{j}}\right)=c_{j} c_{i}^{e_{j}} x^{e_{i} e_{j}}
$$

id est

$$
c_{j}^{e_{i}-1}=c_{i}^{e_{j}-1} .
$$

We thus have $\left(\begin{array}{l}n \\ 2\end{array}\right)$ relations among the $n$ coefficients $c_{i}$. In particular we need to partition the indices as

$$
\{1, \ldots, n\}=E \sqcup O \sqcup S, E=\left\{i: 2 \mid e_{i}\right\}, O=\left\{i: 2 \nmid e_{i}>1\right\}, S=\left\{i: e_{i}=1\right\} .
$$

If $I:=O \sqcup E=\emptyset$ then each such equations are the trivial equality $1=1$ and thus all $c_{i}$ are free. The situation is completely different when $I:=O \sqcup E \neq \emptyset$; in fact,

- for $i \in S$ necessarily $c_{i}= \pm 1$;

- if a prime $p$ divides at least a $c_{j}, j \in I$, then it divides each $c_{i}, i \in I$.

As regards the sign of $c_{i}$ we can say that

- if $E \neq \emptyset$ then

- $c_{i}$ is positive for each $i \in S \cup O$,

- the sign of $c_{i}, i \in E$, is undetermined but all the $c_{i}, i \in E$, have the same sign.

- if $E=\emptyset$ then the sign of $c_{i}, i \in S \cup O$ is undetermined.

For instance 
- for $e_{1}=e_{4}=1, e_{2}=5, e_{3}=3$ we have $S=\{1,4\}, O=\{2,3\}, E=\emptyset$ and

$$
c_{1}^{4}=c_{2}^{0}, c_{1}^{2}=c_{3}^{0}, c_{1}^{0}=c_{4}^{0}, c_{2}^{2}=c_{3}^{4}, c_{2}^{0}=c_{4}^{4}, c_{3}^{0}=c_{4}^{2} ;
$$

whence $c_{1}= \pm 1, c_{4}= \pm 1, c_{2}= \pm c_{3}^{2}$;

- for $e_{1}=e_{4}=1, e_{2}=2, e_{3}=3$ we have $S=\{1,4\}, O=\{3\}, E=\{2\}$, and

$$
c_{1}=c_{2}^{0}, c_{1}^{2}=c_{3}^{0}, c_{1}^{0}=c_{4}^{0}, c_{2}^{2}=c_{3}, c_{2}^{0}=c_{4}, c_{3}^{0}=c_{4}^{2} ;
$$

whence $c_{1}=c_{4}=1, c_{3}=c_{2}^{2}>0$;

- for $e_{1}=1, e_{2}=2, e_{3}=3, S=\{1\} E=\{2\}, O=\{3\}$. Suppose $c_{2}=6$, so both the primes 2 and 3 divide $c_{2}$. From $c_{1}=c_{2}^{0}, c_{1}^{2}=c_{3}^{0}, c_{2}^{2}=c_{3}$ we get $c_{1}=1$ and $c_{3}=36$. We notice that $2 \mid c_{3}$ and $3 \mid c_{3}$, but neither 2 nor 3 divide $c_{1}$;

- for $e_{1}=e_{4}=1, e_{2}=4, e_{3}=8$ we have $S=\{1,4\}, E=\{2,3\}, O=\emptyset$ and

$$
c_{1}^{3}=c_{2}^{0}, c_{1}^{7}=c_{3}^{0}, c_{1}^{0}=c_{4}^{0}, c_{2}^{7}=c_{3}^{3}, c_{2}^{0}=c_{4}^{3}, c_{3}^{0}=c_{4}^{7} .
$$

whence $c_{1}=c_{4}=1, c_{2}=\chi^{3}, c_{3}=\chi^{7}, c_{2} c_{3}>0$.

As regards the values $\left|c_{i}\right|, 1 \leq i \leq n$, setting

$$
\rho:=\sum_{j=1}^{n}\left(e_{j}-1\right)=\sum_{j \in I}\left(e_{j}-1\right), \chi:=\sqrt[\rho]{\prod_{j=1}^{n}\left|c_{j}\right|}
$$

we have

$$
\left|c_{j}\right|=\chi^{e_{j}-1} \text { for each } j \in\{1, \ldots, n\} .
$$

In fact, since if a prime $p$ divides at least a $c_{j}, j \in I$, then it divides each $c_{i}, i \in I$, we can express each $\left|c_{i}\right|, i \in I$, as $\left|c_{i}\right|=p_{1}^{a_{i, 1}} \cdots p_{h}^{a_{i, h}}$ where $p_{1}, \cdots, p_{h}$ are the prime factors of the squarefree associate $\sqrt{\chi}=p_{1} \cdots p_{h}$ of $\chi$.

We have

$$
\left|c_{i}\right|^{e_{j}-1}=\left|c_{j}\right|^{e_{i}-1} \Longrightarrow p^{a_{i}\left(e_{j}-1\right)}=p^{a_{j}\left(e_{i}-1\right)} \Longrightarrow a_{i}\left(e_{j}-1\right)=a_{j}\left(e_{i}-1\right)
$$

whence $a_{i}=a_{j} \Longleftrightarrow e_{i}=e_{j}$ and $a_{i}>a_{j} \Longleftrightarrow e_{j}<e_{i}$.

Thus the $c_{i}$ s with minimal $e_{i}$ minimalize also all $a_{i, l}, 1 \leq l \leq h$.

We moreover have $a_{j, l}=\frac{a_{i, i}\left(e_{j}-1\right)}{\left(e_{i}-1\right)}, 1 \leq l \leq h$.

Therefore $\prod_{j=1}^{n}\left|c_{j}\right|=\prod_{j \in I}\left|c_{j}\right|=\prod_{l=1}^{h} p_{l}^{a_{j, l}}=\prod_{l=1}^{h} p_{l}^{\frac{a_{i, l} \sum_{j=1}^{n}\left(e_{j}-1\right)}{e_{i}-1}}=\prod_{l=1}^{h} p_{l}^{\frac{a_{i, l},-}{i^{\prime}-1}}$ whence

$$
\chi:=\sqrt[\rho]{\prod_{j=1}^{n}\left|c_{j}\right|}=\prod_{l=1}^{h} p_{l}^{\frac{a_{i, l}}{i_{l}-1}}=\prod_{l=1}^{h} p_{l}^{\frac{a_{j, l}}{e_{j}-1}}
$$

and (1-c).

The formula (1-c) allows to reformulate (1-b) as

$$
\left|c_{j}\right|^{e_{i}-1}=\left|c_{i}\right|^{e_{j}-1}=\chi^{\left(e_{i}-1\right)\left(e_{j}-1\right)} .
$$


Note that we have

$$
\begin{aligned}
\chi^{\left(e_{i}^{a_{i}}-1\right)\left(e_{j}^{a_{j}}-1\right)}=\chi^{\left(e_{i}-1\right) \gamma\left(a_{i}, e_{i}\right)\left(e_{j}-1\right) \gamma\left(a_{j}, e_{j}\right)} & =\left|c_{i}\right|^{\left(e_{j}-1\right) \gamma\left(a_{i}, e_{i}\right) \gamma\left(a_{j}, e_{j}\right)}=\left|c_{i}\right|^{\left(e_{j}^{a_{j}}-1\right) \gamma\left(a_{i}, e_{i}\right)} \\
& =\left|c_{j}\right|^{\left(e_{i}-1\right) \gamma\left(a_{i}, e_{i}\right) \gamma\left(a_{j}, e_{j}\right)}=\left|c_{j}\right|^{\left(e_{i}^{a_{i}}-1\right) \gamma\left(a_{j}, e_{j}\right)}
\end{aligned}
$$

and

$$
\left|c_{i}\right|^{\gamma\left(a_{i}, e_{i}\right)}\left|c_{j}\right|^{e_{i}^{a_{i}} \gamma\left(a_{j}, e_{j}\right)}=\left|c_{i}\right|^{e_{j}^{a_{j}} \gamma\left(a_{i}, e_{i}\right)}\left|c_{j}\right|^{\gamma\left(a_{j}, e_{j}\right)}=\chi^{e_{i}^{a_{i}} e_{j}^{a_{j}}-1} .
$$

To avoid cumbersome and useless case-to-case studies, let us simply assume $c_{i}>0$ for each $i$; under this restricted assumption, a series of inductive arguments allow to deduce

$$
Y_{i} * x^{\alpha}=c_{i}^{\alpha} x^{\alpha e_{i}} Y_{i}
$$

$$
\begin{aligned}
& Y_{i} * x^{\alpha}=\left(Y_{i} * x^{\alpha-1}\right) x=c_{i}^{\alpha-1} x^{(\alpha-1) e_{i}}\left(Y_{i} * x\right)=c_{i}^{\alpha-1} x^{(\alpha-1) e_{i}} c_{i} x^{e_{i}} Y_{i}=c_{i}^{\alpha} x^{\alpha e_{i}} Y_{i} . \\
& Y_{j}^{a_{j}} * x^{b_{0}}=c_{j}^{b_{0} \gamma\left(a_{j}, e_{j}\right)} x^{b_{0} e_{j}^{a_{j}}} Y_{j}^{a_{j}} \\
& Y_{j}^{a_{j}} * x^{b_{0}}=Y_{j}\left(Y_{j}^{a_{j}-1} * x^{b_{0}}\right)=Y_{j} *\left(c_{j}^{b_{0} \gamma\left(a_{j}-1, e_{j}\right)} x^{b_{0} e_{j}^{a_{j}-1}} Y_{j}^{a_{j}-1}\right) \\
&=c_{j}^{b_{0} \gamma\left(a_{j}-1, e_{j}\right)}\left(Y_{j} * x^{b_{0} e_{j}^{a_{j}-1}}\right) Y_{j}^{a_{j}-1} \\
&=c_{j}^{b_{0} \gamma\left(a_{j}-1, e_{j}\right)}\left(c_{j}^{b_{0} e_{j}^{a_{j}-1}} x^{\left(b_{0} e_{j}^{a_{j}-1}\right) e_{j}}\right) Y_{j}^{a_{j}} \\
&=c_{j}^{b_{0} \gamma\left(a_{j}, e_{j}\right)} x^{b_{0} e_{j}^{a_{j}}} Y_{j}^{a_{j}} . \\
& c_{j}^{b_{0} \gamma\left(a_{j}, e_{j}\right)} c_{i}^{b_{0} e_{j}^{a_{j}} \gamma\left(a_{i}, e_{i}\right)}=\chi^{b_{0}\left(e_{j}^{a_{j}} e_{i}^{a_{i}}-1\right)}
\end{aligned}
$$

Substituing $c_{j}=\chi^{e_{j}-1}$ and $c_{i}=\chi^{e_{i}-1}$ we get

$$
\begin{aligned}
& c_{j}^{b_{0} \gamma\left(a_{j}, e_{j}\right)} c_{i}^{b_{0} e_{j}^{a_{j}} \gamma\left(a_{i}, e_{i}\right)}=\chi^{\left(e_{j}-1\right) b_{0} \gamma\left(a_{j}, e_{j}\right)} \chi^{\left(e_{i}-1\right) b_{0} e_{j}^{a_{j}} \gamma\left(a_{i}, e_{i}\right)}=\chi^{\left(e_{j}-1\right) b_{0} \frac{1-e_{j}^{a_{j}}}{1-e_{j}}+\left(e_{i}-1\right) b_{0} e_{j}^{a_{j}} \frac{1-e_{i}^{a_{i}}}{1-e_{i}}} \\
& =\chi^{-b_{0}\left(1-e_{j}^{a_{j}}\right)-b_{0} e_{j}^{a_{j}}\left(1-e_{i}^{a_{i}}\right)} \\
& =\chi^{b_{0} e_{j}^{a_{j}}-b_{0}+b_{0} e_{j}^{a_{j}} e_{i}^{a_{i}}-b_{0} e_{j}^{a_{j}}} \\
& =\chi^{b_{0}\left(e_{j}^{a_{j}} e_{i}^{a_{i}}-1\right)} \text {. } \\
& Y_{i}^{a_{i}} Y_{j}^{a_{j}} * x^{b_{0}}=c_{j}^{b_{0} \gamma\left(a_{j}, e_{j}\right)} c_{i}^{b_{0} e_{j}^{a_{j}} \gamma\left(a_{i}, e_{i}\right)} x^{b_{0} e_{j}^{a_{j}} e_{i}^{a_{i}}} Y_{i}^{a_{i}} Y_{j}^{a_{j}}=\chi^{b_{0}\left(e_{j}^{a_{j}} e_{i}^{a_{i}}-1\right)} x^{b_{0} e_{j}^{a_{j}} e_{i}^{a_{i}}} Y_{i}^{a_{i}} Y_{j}^{a_{j}} \\
& Y_{i}^{a_{i}}\left(Y_{j}^{a_{j}} * x^{b_{0}}\right)=Y_{i}^{a_{i}} *\left(c_{j}^{b_{0} \gamma\left(a_{j}, e_{j}\right)} x^{b_{0} e_{j}^{a_{j}}} Y_{j}^{a_{j}}\right)=c_{j}^{b_{0} \gamma\left(a_{j}, e_{j}\right)}\left(Y_{i}^{a_{i}} * x^{b_{0} e_{j}^{a_{j}}}\right) Y_{j}^{a_{j}} \\
& =c_{j}^{b_{0} \gamma\left(a_{j}, e_{j}\right)}\left(c_{i}^{b_{0} e_{j}^{a_{j}} \gamma\left(a_{i}, e_{i}\right)} x^{b_{0} e_{j}^{a_{j}} e_{i}^{a_{i}}} Y_{i}^{a_{i}}\right) Y_{j}^{a_{j}} \\
& =c_{j}^{b_{0} \gamma\left(a_{j}, e_{j}\right)} c_{i}^{b_{0} e_{j}^{a_{j}} \gamma\left(a_{i}, e_{i}\right)} x^{b_{0} e_{j}^{a_{j}} e_{i}^{a_{i}}} Y_{i}^{a_{i}} Y_{j}^{a_{j}} .
\end{aligned}
$$




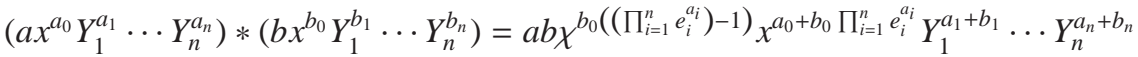

$$
\begin{aligned}
& \left(a x^{a_{0}} Y_{1}^{a_{1}} \cdots Y_{n}^{a_{n}}\right) *\left(b x^{b_{0}} Y_{1}^{b_{1}} \cdots Y_{n}^{b_{n}}\right) \\
& =a x^{a_{0}} Y_{1}^{a_{1}}\left(Y_{2}^{a_{2}} \cdots Y_{n}^{a_{n}} * b x^{b_{0}}\right) Y_{1}^{b_{1}} \cdots Y_{n}^{b_{n}} \\
& =a x^{a_{0}} Y_{1}^{a_{1}}\left(b \chi^{b_{0}\left(\left(\prod_{i=2}^{n} e_{i}^{a_{i}}\right)-1\right)} x^{b_{0} \prod_{i=2}^{n} e_{i}^{a_{i}}}\right) Y_{1}^{b_{1}} Y_{2}^{a_{2}+b_{2}} \cdots Y_{n}^{a_{n}+b_{n}} \\
& =a b x^{b_{0}\left(\left(\prod_{i=2}^{n} e_{i}^{a_{i}}\right)-1\right)} x^{a_{0}}\left(Y_{1}^{a_{1}} * x^{b_{0} \prod_{i=2}^{n} e_{i}^{a_{i}}}\right) Y_{1}^{b_{1}} Y_{2}^{a_{2}+b_{2}} \cdots Y_{n}^{a_{n}+b_{n}} \\
& =a b \chi^{b_{0}\left(\left(\prod_{i=2}^{n} e_{i}^{a_{i}}\right)-1\right)} x^{a_{0}}\left(c_{1}^{b_{0} \prod_{i=2}^{n} e_{i}^{a_{i}} \gamma\left(a_{1}, e_{1}\right)} x^{b_{0}\left(\prod_{i=2}^{n} e_{i}^{a_{i}}\right) e_{1}^{a_{1}}}\right) Y_{1}^{a_{1}+b_{1}} Y_{2}^{a_{2}+b_{2}} \cdots Y_{n}^{a_{n}+b_{n}} \\
& =a b \chi^{b_{0}\left(\left(\prod_{i=2}^{n} e_{i}^{a_{i}}\right)-1\right)} x^{a_{0}}\left(\chi^{\left(e_{1}-1\right) b_{0} \prod_{i=2}^{n} e_{i}^{a_{i}} \frac{1-e_{1}}{1-e_{1}}} x^{b_{0} \prod_{i=1}^{n} e_{i}^{a_{i}}}\right) Y_{1}^{a_{1}+b_{1}} \cdots Y_{n}^{a_{n}+b_{n}} \\
& =a b \chi^{b_{0}\left(\left(\prod_{i=1}^{n} e_{i}^{a_{i}}\right)-1\right)} x^{a_{0}+b_{0} \prod_{i=1}^{n} e_{i}^{a_{i}}} Y_{1}^{a_{1}+b_{1}} \cdots Y_{n}^{a_{n}+b_{n}} .
\end{aligned}
$$

Note that associativity is verified by

$$
\begin{aligned}
& {\left[\left(a x^{a_{0}} Y_{1}^{a_{1}} \cdots Y_{n}^{a_{n}}\right) *\left(b x^{b_{0}} Y_{1}^{b_{1}} \cdots Y_{n}^{b_{n}}\right)\right] *\left(d x^{d_{0}} Y_{1}^{d_{1}} \cdots Y_{n}^{d_{n}}\right)} \\
& =\left[a b x^{b_{0}\left(\left(\prod_{i=1}^{n} e_{i}^{a_{i}}\right)-1\right)} x^{a_{0}+b_{0} \prod_{i=1}^{n} e_{i}^{a_{i}}} Y_{1}^{a_{1}+b_{1}} \cdots Y_{n}^{a_{n}+b_{n}}\right] *\left(d x^{d_{0}} Y_{1}^{d_{1}} \cdots Y_{n}^{d_{n}}\right) \\
& =a b d \chi^{b_{0}\left(\left(\prod_{i=1}^{n} e_{i}^{a_{i}}\right)-1\right)+d_{0}\left(\left(\prod_{i=1}^{n} e_{i}^{a_{i}+b_{i}}\right)-1\right)} \chi^{a_{0}+b_{0}} \prod_{i=1}^{n} e_{i}^{a_{i}}+d_{0} \prod_{i=1}^{n} e_{i}^{a_{i}+b_{i}} Y_{1}^{a_{1}+b_{1}+d_{1}} \cdots Y_{n}^{a_{n}+b_{n}+d_{n}}
\end{aligned}
$$

and

$$
\begin{aligned}
& \left(a x^{a_{0}} Y_{1}^{a_{1}} \cdots Y_{n}^{a_{n}}\right) *\left[\left(b x^{b_{0}} Y_{1}^{b_{1}} \cdots Y_{n}^{b_{n}}\right) *\left(d x^{d_{0}} Y_{1}^{d_{1}} \cdots Y_{n}^{d_{n}}\right)\right] \\
& =\left(a x^{a_{0}} Y_{1}^{a_{1}} \cdots Y_{n}^{a_{n}}\right) *\left[b d \chi^{d_{0}\left(\left(\prod_{i=1}^{n} e_{i}^{b_{i}}\right)-1\right)} x^{b_{0}+d_{0} \prod_{i=1}^{n} e_{i}^{b_{i}}} Y_{1}^{b_{1}+d_{1}} \cdots Y_{n}^{b_{n}+d_{n}}\right]
\end{aligned}
$$

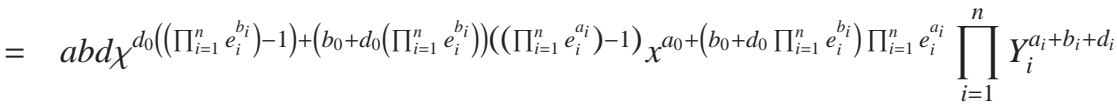

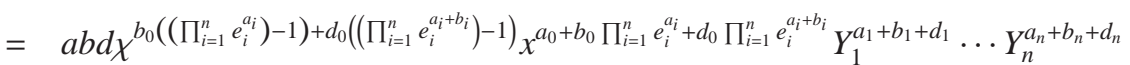

\section{Buchberger Theory}

\section{$2.1 \quad$ Term ordering}

For each $m \in \mathbb{N}$, the free R-module $\mathrm{R}^{m}$ - the canonical basis of which will be denoted $\left\{\mathbf{e}_{1}, \ldots, \mathbf{e}_{m}\right\}-$ is an $(R, R)$-bimodule with basis the set of the terms

$$
\mathcal{T}^{(m)}:=\left\{t \mathbf{e}_{i}: t \in \mathcal{T}, 1 \leq i \leq m\right\} .
$$

If we impose on $\mathcal{T}^{(m)}$ a total ordering $<$, then each $f \in \mathrm{R}^{m}$ has a unique representation as an ordered linear combination of terms $t \in \mathcal{T}^{(m)}$ with coefficients in $R$ :

$$
f=\sum_{i=1}^{s} c\left(f, t_{i}\right) t_{i}: c\left(f, t_{i}\right) \in R \backslash\{0\}, t_{i} \in \mathcal{T}^{(m)}, t_{1}>\cdots>t_{s} .
$$


The support of $f$ is the set $\operatorname{supp}(f):=\{t \mid c(f, t) \neq 0\}$.

W.r.t. $<$ we denote $\mathbf{T}(f):=t_{1}$ the maximal term of $f, \operatorname{lc}(f):=c\left(f, t_{1}\right)$ its leading cofficient and $\mathbf{M}(f):=c\left(f, t_{1}\right) t_{1}$ its maximal monomial.

If we denote, following [44, 45], $\mathrm{M}\left(\mathrm{R}^{m}\right):=\left\{c t \mathbf{e}_{i} \mid c \in R \backslash\{0\}, t \in \mathcal{T}, 1 \leq i \leq m\right\}$, for each $f \in \mathrm{R}^{m} \backslash\{0\}$ the unique finite representation above can be reformulated

$$
f=\sum_{\tau \in \operatorname{supp}(f)} m_{\tau}, m_{\tau}=c(f, \tau) \tau
$$

as a sum of elements of the monomial set $\mathrm{M}\left(\mathrm{R}^{m}\right)$.

While a multivariate Ore extension does not satisfiy commutativity between terms and coefficients,

$$
t \star r=r t \text { for each } r \in R \backslash\{0\}, t \in \mathcal{T},
$$

it however satisfies

$$
\mathbf{M}(t \star r)=\alpha_{t}(r) t, \text { for each } r \in R \backslash\{0\}, t \in \mathcal{T}^{(m)} ;
$$

moreover, while $\mathrm{R}$ is not a monoid ring under the multiplication $\star$, so that in particular we cannot claim $\tau \star \omega \in \mathcal{T}$ for $\tau, \omega \in \mathcal{T}$, however $\tau \star \omega$ satisfies

$$
\mathbf{T}(\tau \star \omega)=\tau \circ \omega
$$

where we have denoted $\circ$ the (commutative) multiplication of $\mathcal{T}$; similarly, for $n \in$ $\mathrm{M}\left(\mathrm{R}^{m}\right)$ and $m_{l}, m_{r} \in \mathrm{M}(\mathrm{R})=\{c t: c \in R \backslash\{0\}, t \in \mathcal{T}\}$ we have $\mathrm{M}\left(m_{l} \star m \star m_{r}\right)=m_{l} * m * m_{r}$.

In conclusion w.r.t. each term ordering $<$ on $\mathcal{T}$ and each $<$-compatible term ordering $<$ on $\mathcal{T}^{(m)}$, id est any well-ordering on $\mathcal{T}^{(m)}$ which satisfies

$$
\omega_{1} \prec \omega_{2} \Longrightarrow \omega_{1} t<\omega_{2} t, t \omega_{1}<t \omega_{2} \text { for each } t \in \mathcal{T}^{(m)}, \omega_{1}, \omega_{2} \in \mathcal{T},
$$

it holds, for each $l, r \in \mathrm{R}$ and $f \in \mathbf{R}^{(m)}$,

$$
\mathbf{T}(l \star f \star r)=\mathbf{T}(l) \circ \mathbf{T}(f) \circ \mathbf{T}(r)
$$

and

$$
\mathbf{M}(l \star f \star r)=\mathbf{M}(\mathbf{M}(l) \star \mathbf{M}(f) \star \mathbf{M}(r))=\mathbf{M}(l) * \mathbf{M}(f) * \mathbf{M}(r) .
$$

As a consequence we trivially have

Corollary 15. If $<$ is a term ordering on $\mathcal{T}$ and $<$ is a $<$-compatible term ordering on $\mathcal{T}^{(m)}$, then, for each $l, r \in \mathrm{R}$ and $f \in \mathbf{R}^{(m)}$,

1. $\mathbf{M}(l \star f)=\mathbf{M}(\mathbf{M}(l) \star \mathbf{M}(f))=\mathbf{M}(l) * \mathbf{M}(f)$;

2. $\mathbf{M}(f \star r)=\mathbf{M}(\mathbf{M}(f) \star \mathbf{M}(r))=\mathbf{M}(f) * \mathbf{M}(r)$;

3. $\mathbf{M}(l \star f \star r)=\mathbf{M}(\mathbf{M}(l) \star \mathbf{M}(f) \star \mathbf{M}(r))=\mathbf{M}(l) * \mathbf{M}(f) * \mathbf{M}(r)$.

4. $\mathbf{T}(l \star f)=\mathbf{T}(l) \circ \mathbf{T}(f)$;

5. $\mathbf{T}(f \star r)=\mathbf{T}(f) \circ \mathbf{T}(r)$;

6. $\mathbf{T}(l \star f \star r)=\mathbf{T}(l) \circ \mathbf{T}(f) \circ \mathbf{T}(r)$. 


\subsection{Gröbner Bases}

In function of a term ordering $<$ on $\mathcal{T}^{(m)}$ which is compatible with a term ordering on $\mathcal{T}$ which, with a slight abuse of notation, we still denote $<$, we denote, for any set $F \subset \mathrm{R}^{m}, \mathbb{I}_{L}(F), \mathbb{I}_{R}(F), \mathbb{I}_{2}(F)$ the left (resp. right, bilateral) module generated by $F$, and

$-\mathbf{T}\{F\}:=\{\mathbf{T}(f): f \in F\} \subset \mathcal{T}^{(m)}$

- $\mathbf{M}\{F\}:=\{\mathbf{M}(f): f \in F\} \subset \mathrm{M}\left(\mathrm{R}^{m}\right)$.

- $\mathbf{T}_{L}(F):=\mathbb{I}_{L}(\mathbf{T}\{F\})=\{\mathbf{T}(\lambda \star f): \lambda \in \mathcal{T}, f \in F\}=\{\lambda \circ \mathbf{T}(f): \lambda \in \mathcal{T}, f \in F\} \subset$ $\mathcal{T}^{(m)}$

- $\mathbf{M}_{L}(F):=\{\mathbf{M}(a \lambda \star f): a \in R \backslash\{0\}, \lambda \in \mathcal{T}, f \in F\}=\{m * \mathbf{M}(f): m \in \mathbf{M}(\mathrm{R}), f \in$ $F\} \subset \mathrm{M}\left(\mathrm{R}^{m}\right)$;

$-\mathbf{T}_{R}(F):=\mathbb{I}_{R}(\mathbf{T}\{F\})=\{\mathbf{T}(f \star \rho): \rho \in \mathcal{T}, f \in F\}=\{\mathbf{T}(f) \circ \rho: \rho \in \mathcal{T}, f \in F\} \subset$ $\mathcal{T}^{(m)}$

- $\mathbf{M}_{R}(F):=\{\mathbf{M}(f \star b \rho): b \in R \backslash\{0\}, \rho \in \mathcal{T}, f \in F\}=\{\mathbf{M}(f) * n: n \in \mathbf{M}(\mathrm{R}), f \in$ $F\} \subset \mathrm{M}\left(\mathrm{R}^{m}\right)$;

$-\mathbf{T}_{2}(F):=\mathbb{I}_{2}(\mathbf{T}\{F\})=\{\mathbf{T}(\lambda \star f \star \rho): \lambda, \rho \in \mathcal{T}, f \in F\}=\{\lambda \circ \mathbf{T}(f) \circ \rho: \lambda, \rho \in$ $\mathcal{T}, f \in F\} \subset \mathcal{T}^{(m)}$

$-\mathbf{M}_{2}(F):=\{\mathbf{M}(a \lambda \star f \star b \rho): a, b \in R \backslash\{0\}, \lambda, \rho \in \mathcal{T}, f \in F\}=\{m * \mathbf{M}(f) * n:$ $m, n \in \mathrm{M}(\mathrm{R}), f \in F\} \subset \mathrm{M}\left(\mathrm{R}^{m}\right)$.

Following an intutition by Weispfenning [52] we further denote

- $\mathbb{I}_{W}(F)$ the restricted module generated by $F$,

$$
\mathbb{I}_{W}(F):=\operatorname{Span}_{R}(a f \star \rho: a \in R \backslash\{0\}, \rho \in \mathcal{T}, f \in F),
$$

$-\mathbf{T}_{W}(F):=\mathbf{T}_{R}(F)$,

$-\mathbf{M}_{W}(F):=\{\mathbf{M}(a f \star \rho): a \in R \backslash\{0\}, \rho \in \mathcal{T}, f \in F\}=\{a \mathbf{M}(f) * \rho: a \in R \backslash\{0\}, \rho \in$ $\mathcal{T}, f \in F\} \subset \mathrm{M}\left(\mathrm{R}^{m}\right)$.

If $R$ is a skew field, for each set $F \subset \mathrm{R}^{m}$ we have

$$
\begin{aligned}
& \mathbf{M}_{L}(F)=\mathbf{M}\left\{\mathbb{I}_{L}(\mathbf{M}\{F\})\right\}=\mathbb{I}_{L}(\mathbf{M}\{F\}) \cap \mathrm{M}\left(\mathrm{R}^{m}\right), \\
& \mathbf{M}_{R}(F)=\mathbf{M}\left\{\mathbb{I}_{R}(\mathbf{M}\{F\})\right\}=\mathbb{I}_{R}(\mathbf{M}\{F\}) \cap \mathbf{M}\left(\mathrm{R}^{m}\right), \\
& \mathbf{M}_{2}(F)=\mathbf{M}\left\{\mathbb{I}_{2}(\mathbf{M}\{F\})\right\}=\mathbb{I}_{2}(\mathbf{M}\{F\}) \cap \mathbf{M}\left(\mathrm{R}^{m}\right) \text {, } \\
& \mathbf{M}_{W}(F)=\mathbf{M}\left\{\mathbb{I}_{W}(\mathbf{M}\{F\})\right\}=\mathbb{I}_{W}(\mathbf{M}\{F\}) \cap \mathbf{M}\left(\mathrm{R}^{m}\right) \text {. }
\end{aligned}
$$

Notation 16. From now on, in order to avoid cumbersome notation and boring repetitions, we will drop the subscripts when it will be clear of which kind of module (left, right, bilateral, restricted) we are discussing. As a consequence, the four statements of (7) will be summarized as

$$
\mathbf{M}(F)=\mathbf{M}\{\mathbb{I}(\mathbf{M}\{F\})\}=\mathbb{I}(\mathbf{M}\{F\}) \cap \mathbf{M}\left(R^{m}\right) .
$$


Similarly, we formulate a (left,right,bilateral,restricted) definition simply for the bilateral case leaving to the reader the task to remove the irrelevant factors.

For instance condition (ii) below is stated for the bilateral case; it would be reformulated:

left case for each $f \in \mathbb{I}(F)$ there are $g \in F, a \in R \backslash\{0\}, \lambda \in \mathcal{T}$ such that $\mathbf{M}(f)=a \lambda * \mathbf{M}(g)=$ $\mathbf{M}(a \lambda \star g)$,

right case for each $f \in \mathbb{I}(F)$ there are $g \in F, b \in R \backslash\{0\}, \rho \in \mathcal{T}$ such that $\mathbf{M}(f)=\mathbf{M}(g) * b \rho=$ $\mathbf{M}(g \star b \rho)$,

restricted case for each $f \in \mathbb{I}(F)$ there are $g \in F, a \in R \backslash\{0\}, \rho \in \mathcal{T}$ such that $\mathbf{M}(f)=a \mathbf{M}(g) * \rho=$ $\mathbf{M}(a g \star \rho)$,

Thus, if $R$ is a skew field, the following conditions are equivalent and can be naturally chosen as definition of Gröbner bases:

1. $\mathbf{M}(\mathbb{I}(F))=\mathbf{M}\{\mathbb{I}(F)\}=\mathbf{M}\{\mathbb{I}(\mathbf{M}\{F\})\}=\mathbb{I}(\mathbf{M}\{F\}) \cap \mathbf{M}\left(\mathrm{R}^{m}\right)$,

2. for each $f \in \mathbb{I}(F)$ there is $g \in F$ such that $\mathbf{M}(g) \mid \mathbf{M}(f)$.

But in general between these statements there is just the implication (2) $\Longrightarrow(1)$.

Thus [39], there are two alternative natural definitions for the concept of Gröbner bases:

- a stronger one which satisfies the following equivalent conditions:

(i). for each $f \in \mathbb{I}(F)$ there is $g \in F$ such that $\mathbf{M}(g) \mid \mathbf{M}(f)$,

(ii). for each $f \in \mathbb{I}(F)$ there are $g \in F, a, b \in R \backslash\{0\}, \lambda, \rho \in \mathcal{T}$ such that $\mathbf{M}(f)=a \lambda * \mathbf{M}(g) * b \rho=\mathbf{M}(a \lambda \star g \star b \rho)$,

(iii). $\mathbf{M}(\mathbb{I}(F))=\mathbf{M}\{\mathbb{I}(F)\}=\mathbf{M}(F)$;

- and a weaker one which satisfies the following equivalent conditions:

(iv). for each $f \in \mathbb{I}(F)$ there are $g_{i} \in F, a_{i}, b_{i} \in R \backslash\{0\}, \lambda_{i}, \rho_{i} \in \mathcal{T}$ for which, denoting $\tau_{i}:=\mathbf{T}\left(g_{i}\right)$, one has

- $\mathbf{T}(f)=\lambda_{i} \circ \mathbf{T}\left(g_{i}\right) \circ \rho_{i}$ for each $i$, and $\operatorname{lc}(f)=\sum_{i} a_{i} \alpha_{\lambda_{i}}\left(\operatorname{lc}\left(g_{i}\right)\right) \alpha_{\lambda_{i} \tau_{i}}\left(b_{i}\right)$

and, equivalently,

- $\mathbf{M}(f)=\sum_{i} a_{i} \lambda_{i} * \mathbf{M}\left(g_{i}\right) * b_{i} \rho_{i}=\sum_{i} \mathbf{M}\left(a_{i} \lambda_{i} \star g_{i} \star b_{i} \rho_{i}\right) ;$

(v). $\mathbf{M}(\mathbb{I}(F))=\mathbf{M}\{\mathbb{I}(F)\}=\mathbf{M}\{\mathbb{I}(\mathbf{M}\{F\})\}=\mathbb{I}(\mathbf{M}\{F\}) \cap \mathbf{M}\left(\mathrm{R}^{m}\right)$;

if moreover $R$ is a skew field $\mathbf{M}(F)=\mathbf{M}\{\mathbb{I}(\mathbf{M}\{F\})\}$ so that conditions (i-v) above are all equivalent and are also equivalent to

(vi). $\mathbf{T}(f)=\lambda \circ \mathbf{T}(g) \circ \rho$ for some $g \in F, \lambda, \rho \in \mathcal{T}$. 
Example 17. Let us now specialize the ring of Example 14 to the case

$$
n=3, e_{1}=2, e_{2}=3, e_{3}=4, \chi=5, c_{1}=5, c_{2}=5^{2}, c_{3}=5^{3}
$$

and remark that

$$
a x^{a_{0}} Y_{1}^{a_{1}} Y_{2}^{a_{2}} Y_{3}^{a_{3}} * b x^{b_{0}} Y_{1}^{b_{1}} Y_{2}^{b_{2}} Y_{3}^{b_{3}}=a b 5^{b_{0}\left(2^{a_{1}} 3^{a_{2}} 4^{a_{3}}-1\right)} x^{a_{0}+b_{0} 2^{a_{1}} 3^{a_{2}} 4^{a_{3}}} Y_{1}^{a_{1}+b_{1}} Y_{2}^{a_{2}+b_{2}} Y_{3}^{a_{3}+b_{3}} .
$$

As a consequence, for each $\left(b_{0}, b_{1}, b_{2}, b_{3}\right),\left(j_{0}, j_{1}, j_{2}, j_{3}\right) \in \mathbb{N}^{4}$

$$
j x^{j_{0}} Y_{1}^{j_{1}} Y_{2}^{j_{2}} Y_{3}^{j_{3}} \in \mathbb{I}_{L}\left(b x^{b_{0}} Y_{1}^{b_{1}} Y_{2}^{b_{2}} Y_{3}^{b_{3}}\right)
$$

if and only if

$$
a_{1}:=j_{1}-b_{1} \geq 0, a_{2}:=j_{2}-b_{2} \geq 0, a_{3}:=j_{3}-b_{3} \geq 0, a_{0}:=j_{0}-b_{0} 2^{a_{1}} 3^{a_{2}} 4^{a_{3}} \geq 0
$$

and $b 5^{b_{0}\left(2^{a_{1}} 3^{a_{2}} 4^{a_{3}}-1\right)} \mid j$.

Note that if we set $y:=5 x$ then for each $\left(b_{1}, b_{2}, b_{3}\right),\left(j_{1}, j_{2}, j_{3}\right) \in \mathbb{N}^{3}$ and $b(y), j(y) \in$ $\mathbb{Z}[y] \subset R$

$$
j(y) Y_{1}^{j_{1}} Y_{2}^{j_{2}} Y_{3}^{j_{3}} \in \mathbb{I}_{L}\left(b(y) Y_{1}^{b_{1}} Y_{2}^{b_{2}} Y_{3}^{b_{3}}\right)
$$

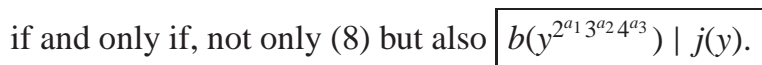

Definition 18. Let I $\subset \mathrm{R}^{m}$ be a (left, right, bilateral, restricted) module and $G \subset \mathrm{I}$.

- $G$ will be called

- a (left, right, bilateral, restricted) weak Gröbner basis (Gröbner basis for short) of I if

$$
\mathbf{M}\{I\}=\mathbf{M}(\mathrm{I})=\mathbf{M}\{\mathbb{I}(\mathbf{M}\{G\})\}=\mathbb{I}(\mathbf{M}\{G\}) \cap \mathrm{M}\left(\mathrm{R}^{m}\right),
$$

id est if $G$ satisfies conditions (iv-v) w.r.t. the module I $=\mathbb{I}(G)$; in particular $\mathbf{M}\{G\}$ generates the (left, right, bilateral, restricted) module $\mathbf{M}(\mathrm{I}) \subset \mathrm{R}^{m}$;

- a (left, right, bilateral, restricted) strong Gröbner basis of I if for each $f \in \mathrm{I}$ there is $g \in G$ such that $\mathbf{M}(g) \mid \mathbf{M}(f)$, id est if $G$ satisfies conditions (i-iii) w.r.t. the module $\mathrm{I}=\mathbb{I}(G)$.

- We say that $f \in \mathrm{R}^{m} \backslash\{0\}$ has

- a left Gröbner representation in terms of $G$ if it can be written as $f=$ $\sum_{i=1}^{u} l_{i} \star g_{i}$, with $l_{i} \in \mathrm{R}, g_{i} \in G$ and $\mathbf{T}\left(l_{i}\right) \circ \mathbf{T}\left(g_{i}\right) \leq \mathbf{T}(f)$ for each $i$;

- a left (weak) Gröbner representation in terms of $G$ if it can be written as $f=\sum_{i=1}^{\mu} a_{i} \lambda_{i} \star g_{i}$, with $a_{i} \in R \backslash\{0\}, \lambda_{i} \in \mathcal{T}, g_{i} \in G$ and $\mathbf{T}\left(\lambda_{i} \star g_{i}\right) \leq$ $\mathbf{T}(f)$ for each $i$;

- a left (strong) Gröbner representation in terms of $G$ if it can be written as $f=\sum_{i=1}^{\mu} a_{i} \lambda_{i} \star g_{i}$, with $a_{i} \in R \backslash\{0\}, \lambda_{i} \in \mathcal{T}, g_{i} \in G$ and

$$
\mathbf{T}(f)=\lambda_{1} \circ \mathbf{T}\left(g_{1}\right)>\lambda_{i} \circ \mathbf{T}\left(g_{i}\right) \text { for each } i
$$


- a right Gröbner representation in terms of $G$ if it can be written as $f=$ $\sum_{i=1}^{u} g_{i} \star r_{i}$, with $r_{i} \in \mathrm{R}, g_{i} \in G$ and $\mathbf{T}\left(g_{i}\right) \circ \mathbf{T}\left(r_{i}\right) \leq \mathbf{T}(f)$ for each $i$;

- a right (weak) Gröbner representation in terms of $G$ if it can be written as $f=\sum_{i=1}^{\mu} g_{i} \star b_{i} \rho_{i}$, with $b_{i} \in R \backslash\{0\}, \rho_{i} \in \mathcal{T}, g_{i} \in G$ and $\mathbf{T}\left(g_{i} \star \rho_{i}\right) \leq \mathbf{T}(f)$ for each $i$;

- a right (strong) Gröbner representation in terms of $G$ if it can be written as $f=\sum_{i=1}^{\mu} g_{i} \star b_{i} \rho_{i}$, with $b_{i} \in R \backslash\{0\}, \rho_{i} \in \mathcal{T}, g_{i} \in G$ and

$$
\mathbf{T}(f)=\mathbf{T}\left(g_{1}\right) \circ \rho_{1}>\mathbf{T}\left(g_{i}\right) \circ \rho_{i} \text { for each } i ;
$$

- a bilateral (weak) Gröbner representation in terms of $G$ if it can be written as $f=\sum_{i=1}^{\mu} a_{i} \lambda_{i} \star g_{i} \star b_{i} \rho_{i}$, with $a_{i}, b_{i} \in R \backslash\{0\}, \lambda_{i}, \rho_{i} \in \mathcal{T}, g_{i} \in G$ and $\mathbf{T}\left(\lambda_{i} \star g_{i} \star \rho_{i}\right) \leq \mathbf{T}(f)$ for each $i$;

- a bilateral (strong) Gröbner representation in terms of $G$ if it can be written as $f=\sum_{i=1}^{\mu} a_{i} \lambda_{i} \star g_{i} \star b_{i} \rho_{i}$, with $a_{i}, b_{i} \in R \backslash\{0\}, \lambda_{i}, \rho_{i} \in \mathcal{T}, g_{i} \in G$ and $\mathbf{T}(f)=\lambda_{1} \circ \mathbf{T}\left(g_{1}\right) \circ \rho_{1}>\lambda_{i} \circ \mathbf{T}\left(g_{i}\right) \circ \rho_{i}$ for each $i$.

- a restricted (weak) Gröbner representation in terms of $G$ if it can be written as $f=\sum_{i=1}^{\mu} a_{i} g_{i} \star \rho_{i}$, with $a_{i} \in R \backslash\{0\}, \rho_{i} \in \mathcal{T}, g_{i} \in G$ and $\mathbf{T}\left(g_{i} \star \rho_{i}\right) \leq \mathbf{T}(f)$ for each $i$;

- a restricted (strong) Gröbner representation in terms of $G$ if it can be written as $f=\sum_{i=1}^{\mu} a_{i} g_{i} \star \rho_{i}$, with $a_{i} \in R \backslash\{0\}, \rho_{i} \in \mathcal{T}, g_{i} \in G$ and $\mathbf{T}(f)=\mathbf{T}\left(g_{1}\right) \circ \rho_{1}>\mathbf{T}\left(g_{i}\right) \circ \rho_{i}$ for each $i$.

- For $f \in \mathrm{R}^{m} \backslash\{0\}, F \subset \mathrm{R}^{m}$, an element $h:=\mathrm{NF}(f, F) \in \mathrm{R}^{m}$ is called a

- (left, right, bilateral, restricted) (weak) normal form of $f$ w.r.t. $F$, if $f-h \in \mathbb{I}(F)$ has a weak Gröbner representation in terms of $F$, and $h \neq 0 \Longrightarrow \mathbf{M}(h) \notin \mathbf{M}\{\mathbb{I}(\mathbf{M}\{F\})\} ;$

- (left, right, bilateral, restricted) strong normal form of $f$ w.r.t. $F$, if $f-h \in \mathbb{I}(F)$ has a strong Gröbner representation in terms of $F$, and $h \neq 0 \Longrightarrow \mathbf{M}(f) \notin \mathbf{M}(F)$.

Proposition 19. (cf. [44 45]) For any set $F \subset \mathrm{R}^{m} \backslash\{0\}$, among the following conditions:

1. $f \in \mathbb{I}(F) \Longleftrightarrow$ it has a (left, right, bilateral, restricted) strong Gröbner representation $f=\sum_{i=1}^{\mu} a_{i} \lambda_{i} \star g_{i} \star b_{i} \rho_{i}$ in terms of $F$ which further satisfies

$$
\mathbf{T}(f)=\mathbf{T}\left(\lambda_{1} \star g_{1} \star \rho_{1}\right)>\cdots>\mathbf{T}\left(\lambda_{i} \star g_{i} \star \rho_{i}\right)>\cdots ;
$$

2. $f \in \mathbb{I}(F) \Longleftrightarrow$ it has a (left, right, bilateral, restricted) strong Gröbner representation in terms of $F$;

3. F is a (left, right, bilateral, restricted) strong Gröbner basis of $\mathbb{I}(F)$; 
4. $f \in \mathbb{I}(F) \Longleftrightarrow$ it has a (left, right, bilateral, restricted) weak Gröbner representation in terms of $F$;

5. F is a (left, right, bilateral, restricted) Gröbner basis of $\mathbb{I}(F)$;

6. $f \in \mathbb{I}(F) \Longleftrightarrow$ it has a (left, right) Gröbner representation in terms of F;

7. for each $f \in \mathrm{R}^{m} \backslash\{0\}$ and any (left, right, bilateral, restricted) strong normal form h of $f$ w.r.t. $F$ we have $f \in \mathbb{I}(F) \Longleftrightarrow h=0$;

8. for each $f \in \mathrm{R}^{m} \backslash\{0\}$ and any (left, right, bilateral, restricted) weak normal form h of $f$ w.r.t. $F$ we have $f \in \mathbb{I}(F) \Longleftrightarrow h=0$;

there are the implications

$$
\begin{array}{ccccccc}
(1) & \Leftrightarrow & (2) & \Rightarrow & (4) & \Leftrightarrow & (6) \\
& \prod & \mathbb{1} & & \mathbb{1} & \mathbb{1} & \\
(7) & \Leftarrow & (3) & \Rightarrow & (5) & \Rightarrow & (8)
\end{array}
$$

If $R$ is a skew field we have also the implication (4) $\Longrightarrow(2)$ and as a consequence also (5) $\Longrightarrow$ (3).

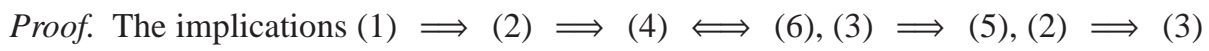
and $(4) \Longrightarrow(5)$ are trivial.

Ad (3) $\Longrightarrow$ (1): for each $f \in \mathbb{I}_{2}(F)$ by assumption there are elements $g \in F, m=$ $a \lambda, n=b \rho \in \mathbf{M}(\mathrm{R})$ such that $\mathbf{M}(f)=\mathbf{M}(m \star g \star n)$. Thus $\mathbf{T}(f)=\mathbf{T}(m \star g \star n)=$ $\lambda \circ \mathbf{T}(g) \circ \rho$ and, denoting $f_{1}:=f-m \star g \star n$, we have $\mathbf{T}\left(f_{1}\right)<\mathbf{T}(f)$ so the claim follows by induction, since $<$ is a well ordering.

Ad (5) $\Longrightarrow$ (4): similarly, for each $f \in \mathbb{I}_{2}(F)$ by assumption there are elements $g_{i} \in F, \mathbf{T}\left(g_{i}\right):=\tau_{i} \mathbf{e}_{i}, m_{i}=a_{i} \lambda_{i}, n_{i}=b_{i} \rho_{i} \in \mathrm{M}(\mathrm{R})$ such that

- $\mathbf{T}(f)=\mathbf{T}\left(\lambda_{i} \star g_{i} \star \rho_{i}\right)=\lambda_{i} \circ \tau_{i} \circ \rho_{i} \mathbf{e}_{l_{i}}$ for each $i$,

$-\operatorname{lc}(f)=\sum_{i} a_{i} \alpha_{\lambda_{i}}\left(\operatorname{lc}\left(g_{i}\right)\right) \alpha_{\lambda_{i} \tau_{i}}\left(b_{i}\right)$.

It is then sufficient to denote $f_{1}:=f-\sum_{i} m_{i} \star g_{i} \star n_{i}$ in order to deduce the claim by induction, since $\mathbf{T}\left(f_{1}\right)<\mathbf{T}(f)$ and $<$ is a well ordering.

$\operatorname{Ad}(4) \Longrightarrow$ (2): let $f \in \mathbb{I}_{2}(F) \backslash\{0\}$; (4) implies the existence of $g \in F, \lambda, \rho \in \mathcal{T}$, such that $\mathbf{T}(f)=\lambda \circ \mathbf{T}(g) \circ \rho$. Then setting $f_{1}:=f-\operatorname{lc}(f)\left(\alpha_{\lambda}(\operatorname{lc}(g))\right)^{-1} \lambda \star g \star \rho$ we deduce the claim by induction, since $\mathbf{T}\left(f_{1}\right)<\mathbf{T}(f)$ and $<$ is a well ordering.

Ad (3) $\Longrightarrow$ (7) and (5) $\Longrightarrow$ (8): either

- $h=0$ and $f=f-h \in \mathbb{I}(F)$ or

- $h \neq 0, \mathbf{M}(h) \notin \mathbf{M}(\mathbb{I}(F)), h \notin \mathbb{I}(F)$ and $f \notin \mathbb{I}(F)$.

Ad (7) $\Longrightarrow$ (2) and (8) $\Longrightarrow$ (4): for each $f \in \mathbb{I}(F)$, its normal form is $h=0$ and $f=f-h$ has a strong (resp.: weak) Gröbner representation in terms of $F$. 
Proposition 20. (Compare [30. Proposition 22.2.10]) If F is a (weak, strong) Gröbner basis of $\mathrm{I}:=\mathbb{I}(F)$, then the following holds:

1. Let $g \in \mathrm{R}^{m}$ be a (weak, strong) normal form of $f$ w.r.t. F. If $g \neq 0$, then

$$
\mathbf{T}(g)=\min \{\mathbf{T}(h): h-f \in \mathbb{I}(F)\} .
$$

2. Let $f, f^{\prime} \in \mathrm{R}^{m} \backslash \mathrm{I}$ be such that $f-f^{\prime} \in \mathrm{I}$. Let $g$ be a (weak, strong) normal form of $f$ w.r.t. $F$ and $g^{\prime}$ be a (weak, strong) normal form of $f^{\prime}$ w.r.t. $F$. Then

- $\mathbf{T}(g)=\mathbf{T}\left(g^{\prime}\right)=: \tau$ and

$$
-\operatorname{lc}(g)-\operatorname{lc}\left(g^{\prime}\right) \in \mathrm{I}_{\tau}:=\{\operatorname{lc}(f): f \in \mathrm{I}, \mathbf{T}(f)=\tau\} \cup\{0\} \subset R .
$$

Proof.

1. Let $h \in \mathrm{R}^{m}$ be such that $h-f \in \mathrm{I}$; then $h-g \in \mathrm{I}$ and $\mathbf{M}(h-g) \in \mathbf{M}\{\mathrm{l}\}$. If $\mathbf{T}(g)>\mathbf{T}(h)$ then $\mathbf{M}(h-g)=\mathbf{M}(g) \notin \mathbf{M}\{l\}$, giving a contradiction.

2. The assumption implies that $f-g^{\prime} \in \mathrm{I}$ so that, by the previous result, $\mathbf{T}(g) \leq$ $\mathbf{T}\left(g^{\prime}\right)$. Symmetrically, $f^{\prime}-g \in \mathrm{I}$ and $\mathbf{T}\left(g^{\prime}\right) \leq \mathbf{T}(g)$. Therefore $\mathbf{T}(g)=\mathbf{T}\left(g^{\prime}\right)=\tau$; morevoer, either

$$
\text { - } \mathbf{T}\left(g-g^{\prime}\right)<\tau \text { and } \mathbf{M}(g)=\mathbf{M}\left(g^{\prime}\right) \text { so that } \operatorname{lc}(g)=\operatorname{lc}\left(g^{\prime}\right) \text { or }
$$

- $\mathbf{T}\left(g-g^{\prime}\right)=\tau$ and $\mathbf{M}\left(g-g^{\prime}\right)=\mathbf{M}(g)-\mathbf{M}\left(g^{\prime}\right)=\left(\operatorname{lc}(g)-\operatorname{lc}\left(g^{\prime}\right)\right) \tau$; thus, since $g-g^{\prime} \in \mathrm{I}, \operatorname{lc}(g)-\operatorname{lc}\left(g^{\prime}\right) \in \mathrm{I}_{\tau}$.

\subsection{Canonical forms (skew field case)}

If $R:=\mathbb{K}$ is a skew field, for any set $F \subset \mathrm{R}^{m}$ we denote $\mathbf{N}(F)$ the (left, right, bilateral, restricted) order module $\mathbf{N}(F):=\mathcal{T}^{(m)} \backslash \mathbf{T}(F)$ and $\mathbb{K}[\mathbf{N}(F)]$ the (left, right, bilateral, restricted) $\mathbb{K}$-module $\mathbb{K}[\mathbf{N}(F)]:=\operatorname{Span}_{\mathbb{K}}(\mathbf{N}(F)$ ).

Definition 21. For any (left, right, bilateral, restricted) module $I \subset \mathrm{R}^{m}$, the order module $\mathbf{N}(I):=\mathcal{T}^{(m)} \backslash \mathbf{T}\{\mid\}$ is called the escalier of $\mathrm{I}$.

We easily obtain the notion, the properties and the computational algorithm (Figure 1) of (left, right, bilateral, restricted) canonical forms:

Lemma 22. (cf. [30, Lemma 22.2.12]) Let $\mathrm{I} \subset \mathrm{R}^{m}$ be a (left, right, bilateral, restricted) module. If $R=\mathbb{K}$ is a skew field and denoting $\mathrm{A}$ the (left, right,bilateral, restricted) module $\mathrm{A}:=\mathrm{R}^{m} / \mathrm{I}$ it holds

1. $\mathrm{R}^{m} \cong \mathrm{I} \oplus \mathbb{K}[\mathbf{N}(\mathrm{I})]$;

2. $\mathrm{A} \cong \mathbb{K}[\mathbf{N}(\mathrm{I})]$; 
Figure 1: Canonical Form Algorithms

$\left(g, \sum_{i=1}^{\mu} c_{i} \lambda_{i} \star g_{i}\right):=\operatorname{LeftCanonicalForm}(f, G)$

where

$G$ is the left Gröbner basis of the left module $\mathrm{I} \subset \mathrm{R}^{m}$,

$f \in \mathrm{R}^{m}, g \in \mathbb{K}[\mathbf{N}(\mathrm{I})], c_{i} \in \mathbb{K} \backslash\{0\}, \lambda_{i} \in \mathcal{T}, g_{i} \in G$,

$f-g=\sum_{i=1}^{\mu} c_{i} \lambda_{i} \star g_{i}$ is a left strong Gröbner representation in terms of $G$,

$\mathbf{T}(f-g)=\lambda_{1} \circ \mathbf{T}\left(g_{1}\right)>\lambda_{2} \circ \mathbf{T}\left(g_{2}\right)>\cdots>\lambda_{\mu} \circ \mathbf{T}\left(g_{\mu}\right)$.

$h:=f, i:=0, g:=0$,

While $h \neq 0$ do

$\% \% f=g+\sum_{j=1}^{i} c_{j} \lambda_{j} \star g_{j}+h$,

$\% \% \mathbf{T}(f-g) \geq \mathbf{T}(h)$

$\% \% i>0 \Longrightarrow \mathbf{T}(f-g)=\lambda_{1} \circ \mathbf{T}\left(g_{1}\right)>\lambda_{2} \circ \mathbf{T}\left(g_{2}\right)>\cdots>\lambda_{i} \circ \mathbf{T}\left(g_{i}\right)>\mathbf{T}(h) ;$

If $\mathbf{T}(h) \in \mathbf{T}_{L}(G) \mathbf{d o}$

Let $\lambda \in \mathcal{T}, \gamma \in G: \lambda \circ \mathbf{T}(\gamma)=\mathbf{T}(h)$

$i:=i+1, c_{i}:=\operatorname{lc}(h) \alpha_{\lambda}(\operatorname{lc}(\gamma))^{-1}, \lambda_{i}:=\lambda, g_{i}:=\gamma, h:=h-c_{i} \lambda_{i} g_{i}$.

Else

$\% \% \mathbf{T}(h) \in \mathbf{N}(\mathrm{I})$

$g:=g+\mathbf{M}(h), h:=h-\mathbf{M}(h)$

$\left(g, \sum_{i=1}^{\mu} g_{i} \star d_{i} \rho_{i}\right):=$ RightCanonicalForm $(f, G)$

where

$G$ is the right Gröbner basis of the right module $\mathrm{I} \subset \mathrm{R}^{m}$,

$f \in \mathrm{R}^{m}, g \in \mathbb{K}[\mathbf{N}(\mathrm{I})], d_{i} \in \mathbb{K} \backslash\{0\}, \rho_{i} \in \mathcal{T}, g_{i} \in G$,

$f-g=\sum_{i=1}^{\mu} g_{i} \star d_{i} \rho_{i}$ is a right strong Gröbner representation in terms of $G$,

$\mathbf{T}(f-g)=\mathbf{T}\left(g_{1}\right) \circ \rho_{1}>\mathbf{T}\left(g_{2}\right) \circ \rho_{2}>\cdots>\mathbf{T}\left(g_{\mu}\right) \circ \rho_{\mu}$.

$h:=f, i:=0, g:=0$,

While $h \neq 0$ do

If $\mathbf{T}(h) \in \mathbf{T}_{R}(G) \mathbf{d o}$

Let $\rho \in \mathcal{T}, \gamma \in G: \mathbf{T}(\gamma) \circ \rho=\mathbf{T}(h)$

$i:=i+1, d_{i}:=\alpha_{\mathbf{T}(\gamma)}^{-1}\left(\operatorname{lc}(h) \operatorname{lc}(\gamma)^{-1}\right), \rho_{i}:=\rho, g_{i}:=\gamma$,

$h:=h-g_{i} \star d_{i} \rho_{i}$.

Else

$g:=g+\mathbf{M}(h), h:=h-\mathbf{M}(h)$ 
Figure 1 (cont.): Canonical Form Algorithms

$\left(g, \sum_{i=1}^{\mu} c_{i} \lambda_{i} \star g_{i} \star \rho_{i}\right):=$ BilateralCanonicalForm $(f, G)$

where

$G$ is the bilateral Gröbner basis of the bilateral module $\mathrm{I} \subset \mathrm{R}^{m}$,

$f \in \mathrm{R}^{m}, g \in \mathbb{K}[\mathbf{N}(\mathrm{I})], c_{i} \in \mathbb{K} \backslash\{0\}, \lambda_{i}, \rho_{i} \in \mathcal{T}, g_{i} \in G$,

$f-g=\sum_{i=1}^{\mu} c_{i} \lambda_{i} \star g_{i} \star \rho_{i}$ is a bilateral strong Gröbner representation in terms of $G$,

$\mathbf{T}(f-g)=\lambda_{1} \circ \mathbf{T}\left(g_{1}\right) \circ \rho_{1}>\lambda_{2} \circ \mathbf{T}\left(g_{2}\right) \circ \rho_{2}>\cdots>\lambda_{\mu} \circ \mathbf{T}\left(g_{\mu}\right) \circ \rho_{\mu}$.

$h:=f, i:=0, g:=0$,

While $h \neq 0$ do

If $\mathbf{T}(h) \in \mathbf{T}_{2}(G) \mathbf{d o}$

Let $\lambda, \rho \in \mathcal{T}, \gamma \in G: \lambda \circ \mathbf{T}(\gamma) \circ \rho=\mathbf{T}(h)$

$i:=i+1, c_{i}:=\operatorname{lc}(h) \alpha_{\lambda}(\operatorname{lc}(\gamma))^{-1}, \lambda_{i}:=\lambda, \rho_{i}:=\rho, g_{i}:=\gamma$,

$h:=h-c_{i} \lambda_{i} \star g_{i} \star \rho_{i}$.

Else

$g:=g+\mathbf{M}(h), h:=h-\mathbf{M}(h)$

$\left(g, \sum_{i=1}^{\mu} c_{i} g_{i} \star \rho_{i}\right):=$ RestrictedCanonicalForm $(f, G)$

where

$G$ is the restricted Gröbner basis of the restricted module $\mathrm{I} \subset \mathrm{R}^{m}$,

$f \in \mathrm{R}^{m}, g \in \mathbb{K}[\mathbf{N}(\mathrm{I})], c_{i} \in \mathbb{K} \backslash\{0\}, \rho_{i} \in \mathcal{T}, g_{i} \in G$,

$f-g=\sum_{i=1}^{\mu} c_{i} g_{i} \star \rho_{i}$ is a restricted strong Gröbner representation in terms of $G$,

$\mathbf{T}(f-g)=\mathbf{T}\left(g_{1}\right) \circ \rho_{1}>\mathbf{T}\left(g_{2}\right) \circ \rho_{2}>\cdots>\mathbf{T}\left(g_{\mu}\right) \circ \rho_{\mu}$.

$h:=f, i:=0, g:=0$,

While $h \neq 0$ do

If $\mathbf{T}(h) \in \mathbf{T}_{W}(G)$ do

Let $\rho \in \mathcal{T}, \gamma \in G: \mathbf{T}(\gamma) \circ \rho=\mathbf{T}(h)$

$i:=i+1, c_{i}:=\operatorname{lc}(h) \operatorname{lc}(\gamma)^{-1}, \rho_{i}:=\rho, g_{i}:=\gamma$,

$h:=h-c_{i} g_{i} \star \rho_{i}$.

Else

$g:=g+\mathbf{M}(h), h:=h-\mathbf{M}(h)$ 
3. for each $f \in \mathrm{R}^{m}$, there is a unique

$$
g:=\operatorname{Can}(f, \mathrm{I})=\sum_{t \in \mathbf{N}(\mathrm{I})} \gamma(f, t,<) t \in \mathbb{K}[\mathbf{N}(\mathrm{I})]
$$

such that $f-g \in \mathrm{I}$.

Moreover:

(a) $\operatorname{Can}\left(f_{1}, \mathrm{I}\right)=\operatorname{Can}\left(f_{2}, \mathrm{I}\right) \Longleftrightarrow f_{1}-f_{2} \in \mathrm{I}$;

(b) $\operatorname{Can}(f, \mathrm{I})=0 \Longleftrightarrow f \in \mathrm{I}$.

4. For each $f \in \mathrm{R}^{m}, f-\operatorname{Can}(f, \mathrm{I})$ has a (left, right, bilateral, restricted) strong Gröbner representation in terms of any Gröbner basis.

Definition 23. (cf. [30, Definition 22.2.13]) For each $f \in \mathrm{R}^{m}$ the unique element

$$
g:=\operatorname{Can}(f, \mathrm{I}) \in \mathbb{K}[\mathbf{N}(\mathrm{I})]
$$

such that $f-g \in \mathrm{I}$ will be called the (left, right, bilateral, restricted) canonical form of $f$ w.r.t. I.

Corollary 24. (cf. [30, Corollary 22.3.14]) If $R=\mathbb{K}$ is a skew field, there is a unique set $G \subset$ I such that

- $\mathbf{T}\{G\}$ is an irredundant basis of $\mathbf{T}(\mathrm{I})$;

- for each $g \in G, \operatorname{lc}(g)=1$;

- for each $g \in G, g=\mathbf{T}(g)-\operatorname{Can}(\mathbf{T}(g), \mathrm{l})$.

$G$ is called the (left, right, bilateral, restricted) reduced Gröbner basis of I.

Note that the algorithm described for right canonical forms is assuming that each $\alpha_{i}$ is an automorphism; alternatively we can assume that $\mathrm{R}$ is given as a right $R$-module in which case the theory can be developped symmetrically.

\section{Szekeres Theory}

Let $\mathrm{I} \subset \mathrm{R}^{m}$ be a (left-bilateral) module; if we denote for each $\tau \in \mathcal{T}^{(m)}, \mathrm{I}_{\tau}$ the additive group

$$
\mathrm{I}_{\tau}:=\{\operatorname{lc}(f): f \in \mathrm{I}, \mathbf{T}(f)=\tau\} \cup\{0\} \subset R,
$$

$\mathfrak{J}:=\left\{I_{\tau}: \tau \in \mathcal{T}^{(m)}\right\}$ and, for each ideal $\mathfrak{a} \subset R, T_{\mathfrak{a}}$ and $L_{\mathfrak{a}}$ the sets

$$
T_{\mathfrak{a}}:=\left\{\tau \in \mathcal{T}^{(m)}: \mathrm{I}_{\tau} \supseteq \mathfrak{a}\right\} \subset \mathcal{T}^{(m)} \text { and } L_{\mathfrak{a}}:=\left\{\tau \in \mathcal{T}^{(m)}: \mathrm{I}_{\tau}=\mathfrak{a}\right\} \subset \mathcal{T}^{(m)},
$$

we have

1. for each $\tau \in \mathcal{T}^{(m)}, \mathrm{I}_{\tau} \subset R$ is a left ideal; 
2. for each ideals $\mathfrak{a}, \mathfrak{b} \subset R, \mathfrak{a} \subset \mathfrak{b} \Longrightarrow T_{\mathfrak{a}} \supset T_{\mathfrak{b}}$;

3. $T_{\mathfrak{a}}=\bigsqcup_{\mathfrak{b} \supseteq \mathfrak{a}} L_{\mathfrak{b}}, L_{\mathfrak{a}}=T_{\mathfrak{a}} \backslash \bigcup_{\mathfrak{b} \mathfrak{a} \mathfrak{a}} T_{\mathfrak{b}}$;

4. for terms $\tau, \omega \in \mathcal{T}^{(m)}, \tau \mid \omega \Longrightarrow \mathrm{I}_{\tau} \subset \mathrm{I}_{\omega}$;

5. for each ideal $\mathfrak{a} \subset R, T_{\mathfrak{a}} \subset \mathcal{T}^{(m)}$ is a right semigroup module.

If $R$ is a skew field, the situation is quite trivial: for any ideal I we have

$$
\mathfrak{J}=\{(0), R\}, T_{R}=L_{R}=\mathbf{T}(\mathrm{I}), T_{(0)}=\mathcal{T}^{(m)}, L_{(0)}=\mathcal{T}^{(m)} \backslash \mathbf{T}(\mathrm{I}) .
$$

Szekeres notation is related with a pre-Buchberger construction of "canonical" ideals for the case of polynomial rings $R\left[Y_{1}, \ldots, Y_{n}\right]$ over a PID $R$.

In connection recall that [13, 14] a not necessarily commutative ring $R$ is called a (left, right, bilateral) Bézout ring if every finitely generated (left, right, bilateral) ideal is principal and is called a Bézout domain if it is both a Bézout ring and is a domain, and remark that, if $R$ is a noetherian (left, bilateral) Bézout ring, then for each $\tau \in \mathcal{T}^{(m)}$, there is a value $c_{\tau} \in R$ satisfying $\mathrm{I}_{\tau}=\mathbb{I}\left(c_{\tau}\right)$.

Definition 25. With the present notation, we call Szekeres ideal each ideal $I_{\tau} \subset R$ and Szekeres level each set $L_{\mathfrak{a}} \subset \mathcal{T}^{(m)}$, Szekeres semigroup each semigroup $T_{\mathfrak{a}} \subset \mathcal{T}^{(m)}$.

Finally, if $R$ is a noetherian left Bézout ring we call Szekeres generator each value $c_{\tau} \in R$ satisfying $\mathrm{I}_{\tau}=\mathbb{I}_{L}\left(c_{\tau}\right)$.

Note that if $R$ is a noetherian Bézout ring, we have,

$$
\omega\left|\tau \Longrightarrow c_{\tau}\right|_{L} \alpha_{\lambda}\left(c_{\omega}\right) \text { for each } \lambda, \rho \in \mathcal{T} \text { s.t. } \tau=\lambda \circ \omega \circ \rho .
$$

Proposition 26 (Szekeres). [50] Let $R$ be a noetherian left Bézout ring and $\mathrm{I} \subset \mathrm{R}^{m}$ be a (left,bilateral) module. Denote

$$
\mathrm{T}:=\left\{\tau \in \mathcal{T}^{(m)} \text { s.t. } c_{\tau} \notin \mathbb{I}\left(\alpha_{\lambda}\left(c_{\omega}\right), \omega \in \mathcal{T}^{(m)}, \lambda, \rho \in \mathcal{T}, \tau=\lambda \circ \omega \circ \rho\right)\right\} \subset \mathcal{T}^{(m)}
$$

and fix, for each $\tau \in \mathrm{T}$, any element $f_{\tau} \in \mathrm{I}$ such tha $1 \mathbf{M}\left(f_{\tau}\right)=c_{\tau} \tau$.

Then the basis $S_{w}:=\left\{f_{\tau}\right.$ s.t. $\left.\tau \in \mathrm{T}\right\}$ is a left/bilateral weak Gröbner basis of $\mathrm{I}$.

Proof. For each $f \in \mathrm{I}$, denoting $\tau:=\mathbf{T}(f)$ we have $\operatorname{lc}(f) \in \mathbb{I}_{L}\left(c_{\tau}\right)$ and $\operatorname{lc}(f)=d c_{\tau}$ for suitable $d \in R \backslash\{0\}$. Thus if $\tau \in \mathrm{T}$ we have $\mathbf{M}(f)=d \mathbf{M}\left(f_{\tau}\right)$; if, instead, $\tau \notin \mathrm{T}$ there are suitable $d_{i}, \in R \backslash\{0\}, \omega_{i} \in \mathrm{T} \subset \mathcal{T}^{(m)}, \lambda_{i}, \rho_{i} \in \mathcal{T}$ for which $\lambda_{i} \circ \omega_{i} \circ \rho_{i}=\tau$ and $c_{\tau}=\sum_{i} d_{i} \alpha_{\lambda_{i}}\left(c_{\omega_{i}}\right)$ so that

$$
\begin{aligned}
\mathbf{M}(f)=d c_{\tau} \tau & =d\left(\sum_{i} d_{i} \alpha_{\lambda_{i}}\left(c_{\omega_{i}}\right) \lambda_{i} \circ \omega_{i} \circ \rho_{i}\right) \\
& =\sum_{i}\left(d d_{i} \lambda_{i}\right) \cdot\left(c_{\omega_{i}} \omega_{i}\right) \cdot \rho_{i} \\
& =\sum_{i}\left(d d_{i} \lambda_{i}\right) * \mathbf{M}\left(f_{\omega_{i}}\right) * \rho_{i} .
\end{aligned}
$$

\footnotetext{
${ }^{1}$ Of course for the extreme case $\mathrm{I}_{\tau}=(0)$ so that $c_{\tau}=0$, we have $f_{\tau}:=0$.
} 
Remark 27. Remark that in the case in which each endomorphism $\alpha_{\tau}, \tau \in \mathcal{T}^{(m)}$, is an automorphism, we can consider also right modules I to which we can associate

$$
\mathrm{I}_{\tau}=\{\operatorname{lc}(f): f \in \mathrm{I}, \mathbf{T}(f)=\tau\} \cup\{0\}
$$

which are right ideals themselves; in fact if we represent $f \in \mathrm{R}^{m}$ as (see Remark 3) $f=\sum_{i=1}^{n} Y^{i} \bar{a}_{i}$ and we denote ${ }_{\tau}$ l the right ideal

$$
{ }_{\tau} \mid:=\{c \in R: \tau c \in \mathbf{M}\{1\}\} \cup\{0\} \subset R
$$

then $\mathrm{I}_{\tau}$ is the right ideal $\alpha_{\tau}\left({ }_{\tau} \mathrm{l}\right)$.

However, in this setting, Szekeres Theory can be built more easily by considering the ideals ${ }_{\tau}$ l obtained through the right representation of Remark 3 and adapting to them the results reported above.

Remark that if an endomorphism $\alpha_{\tau}$ is not invertible, in general $\mathrm{I}_{\tau}$ is not an ideal but just an additive group.

Finally note that for restricted modules, one apply verbatim, the classical Szekeres theory and subistitute in the results above each instance of $\alpha_{\lambda}\left(c_{\omega}\right), \tau=\lambda \circ \omega \circ \rho$ with $c_{\omega}, \tau=\omega \circ \rho$

Example 28. In the Ore extension

$$
\mathrm{R}:=R[Y ; \alpha], R=\mathbb{Z}_{2}[x] \text { where } \alpha: R \rightarrow R: x \mapsto x^{2}
$$

we can consider, as a left module, the two-sided ideal $\mathbb{I}_{2}(x)=\mathbb{I}_{L}\left\{x Y^{i}: i \in \mathbb{N}\right\}$; we thus have

$$
\mathrm{I}_{\tau}=\mathbb{I}(x) \subset R \text { for each } \tau \in\left\{Y^{i}, i \geq 0\right\},
$$

so that, setting $\mathfrak{a}:=\mathbb{I}(x) \subset R$, it holds $\mathfrak{I}=\{\mathfrak{a}\}, T_{\mathfrak{a}}=L_{\mathfrak{a}}=\left\{Y^{i}: i \in \mathbb{N}\right\}$, and $S_{w}=\left\{x Y^{i}\right.$ : $i \in \mathbb{N}\}$ is both a weak and a strong Gröbner basis of $\mathbb{I}_{L}(x)$.

For the right ideal $\mathbb{I}_{R}(x Y)$ the sets $\mathrm{I}_{\tau}$ are not ideals; we have, e.g.

$$
\mathrm{I}_{Y^{i}}=\left\{x \phi\left(x^{e^{i}}\right) \mid \phi(x) \in k[x]\right\} .
$$

\section{Zacharias canonical representation}

Following Zacharias approach to Buchberger Theory [53], if each module $\mathrm{I} \subset \mathrm{R}^{m}$ has a groebnerian property, necessarily the same property must be satisfied at least by the modules $\mathrm{I} \subset R^{m} \subset \mathrm{R}^{m}$ and thus such property in $R^{m}$ can be used to device a procedure granting the same property in $R^{m}$. The most elementary application of Zacharias approach is the generalization of the property of canonical forms from the case in which $R=\mathbb{K}$ is a skew field to the general case: all we need is an effective notion of canonical forms for modules in $R$ :

Definition 29 (Zacharias). [53] A ring $R$ is said to have canonical representatives if there is an algorithm which, given an element $c \in R^{m}$ and a (left,bilateral, right) module $\mathrm{J} \subset R^{m}$, computes a unique element $\operatorname{Rep}(c, \mathrm{~J})$ such that

$$
-c-\boldsymbol{R e p}(c, \mathrm{~J}) \in \mathrm{J},
$$


$-\boldsymbol{\operatorname { R e p }}(c, \mathrm{~J})=0 \Longleftrightarrow c \in \mathrm{J}$.

The set

$$
\boldsymbol{Z a c h}\left(R^{m} / J\right):=\operatorname{Rep}(J):=\left\{\boldsymbol{R e p}(c, J): c \in R^{m}\right\} \cong R^{m} / \mathrm{J}
$$

is called the canonical Zacharias representation of the module $R^{m} / \mathrm{J}$.

Remark that, for each $c, d \in R^{m}$ and each module $\mathrm{J} \subset R^{m}$, we have

$$
c-d \in \mathrm{J} \Longleftrightarrow \boldsymbol{\operatorname { R e p }}(c, \boldsymbol{J})=\operatorname{Rep}(d, \mathrm{~J}) .
$$

Using Szekeres notation for a (left, right, bilateral) module $\mathrm{I} \subset \mathrm{R}^{m}$ we obtain

- the partition $\mathcal{T}^{(m)}=\mathbf{L}(\mathrm{I}) \sqcup \mathbf{R}(\mathrm{I}) \sqcup \mathbf{N}(\mathrm{I})$ of $\mathcal{T}^{(m)}$ where

$$
\begin{aligned}
& -\mathbf{N}(\mathrm{I}):=\mathbf{L}_{(0)}=\left\{\omega \in \mathcal{T}^{(m)}: \mathrm{I}_{\omega}=(0)\right\}, \\
& -\mathbf{L}(\mathrm{I}):=\mathbf{L}_{R}=\left\{\omega \in \mathcal{T}^{(m)}: \mathrm{I}_{\omega}=R\right\}, \\
& -\mathbf{R}(\mathrm{I}):=\left\{\omega \in \mathcal{T}^{(m)}: \mathrm{I}_{\omega} \notin\{(0), R\}\right\} ;
\end{aligned}
$$

- the canonical Zacharias representation

$$
\begin{aligned}
\operatorname{Zach}\left(\mathrm{R}^{m} / \mathrm{l}\right):=\operatorname{Rep}(\mathrm{I}):=\left\{\boldsymbol{\operatorname { R e p }}(c, \mathrm{l}): c \in \mathrm{R}^{m}\right\} & =\bigoplus_{\mathfrak{a} \in \mathcal{I}} \bigoplus_{\tau \in L_{\mathfrak{a}}} \operatorname{Rep}(\mathfrak{a}) \tau \\
& =\bigoplus_{\tau \in \mathcal{T}^{(m)}} \operatorname{Rep}\left(\mathrm{I}_{\tau}\right) \tau \\
& =\bigoplus_{\tau \in \mathcal{T}^{(m)}} \operatorname{Zach}\left(R / \mathrm{I}_{\tau}\right) \tau \cong \mathrm{R}^{m} / \mathrm{I}
\end{aligned}
$$

of the module $\mathrm{R}^{m} / \mathrm{I}$.

If $R$ has canonical representatives and there is an algorithm ( $c f$. Definition 42 (c), (e)) which, given an element $c \in R^{m}$ and a (left, right, bilateral) module $\mathrm{J} \subset R^{m}$ computes the unique canonical representative $\operatorname{Rep}(c, \mathrm{~J})$, an easy adaptation of Figure 1 allows to extend, from the field coefficients case to the Zacharias ring [53, 31] coefficients case, the notion of canonical forms, the algorithm (Figure 2) for computing them and their characterizing properties:

Lemma 30. If $R$ has canonical representatives, also $R$ has canonical representatives.

With the present notation and denoting $\mathrm{A}$ the (left, right, bilateral) module $\mathrm{A}:=$ $\mathrm{R}^{m} /$ it holds:

1. $\mathrm{R}^{m} \cong \mathrm{I} \oplus \boldsymbol{\operatorname { R e p }}(\mathrm{I})$;

2. $A \cong \operatorname{Rep}(I)$;

3. for each $f \in \mathrm{R}^{m}$, there is a unique (left, right, bilateral) canonical form of $f$

$$
g:=\operatorname{Can}(f, \mathrm{I})=\sum_{\mathrm{a} \in \mathfrak{I}} \sum_{\tau \in L_{\mathrm{a}}} \gamma(f, \tau, \mathrm{I},<) \tau \in \operatorname{Rep}(\mathrm{I}), \quad \gamma(f, \tau, \mathrm{I},<) \in \operatorname{Rep}\left(\mathrm{I}_{\tau}\right),
$$

such that 
$-f-g \in \mathrm{I}$,

- $\gamma(f, \tau, \mathrm{I},<)=\boldsymbol{\operatorname { R e p }}\left(\gamma(f, \tau, \mathrm{I},<), \mathrm{I}_{\tau}\right) \in \boldsymbol{\operatorname { R e p }}\left(\mathrm{I}_{\tau}\right)$, for each $\tau \in \mathcal{T}^{(m)}$.

Moreover:

(a) $\operatorname{Can}\left(f_{1}, \mathrm{I}\right)=\operatorname{Can}\left(f_{2}, \mathrm{I}\right) \Longleftrightarrow f_{1}-f_{2} \in \mathrm{I}$;

(b) $\operatorname{Can}(f, \mathrm{I})=0 \Longleftrightarrow f \in \mathrm{I}$;

4. for each $f \in \mathrm{R}^{m}, f-\operatorname{Can}(f$, I) has a (left, right, bilateral) (weak, strong) Gröbner representation in terms of any (weak, strong) Gröbner basis.

\section{Möller's Lifting Theorem}

\subsection{Left case}

The validity of Eqs. (5) and (6) allows to intoduce the groebnerian terminology and, as in the standard theory of commutative polynomial rings over a field [30, § 21.1-2] or a Zacharias ring [53], the ability of imposing a $\mathcal{T}^{(m)}$-valuation on modules over $\mathrm{R}$ and its associated graded Ore extension $S:=G(\mathrm{R})$ (see Remark 10).

The only twist w.r.t. the classical theory is that there the ring was coinciding with its associated graded ring; here they coincide as sets and as left $R$-modules, but as rings have two different multiplications.

Consequently, denoting by $\star$ the one of $\mathrm{R}$ and by $*$ the one of $\mathrm{S}$, given a finite basis

$$
F:=\left\{g_{1}, \ldots, g_{u}\right\} \subset \mathbf{R}^{m}, g_{i}=\mathbf{M}\left(g_{i}\right)-p_{i}=: c_{i} \tau_{i} \mathbf{e}_{l_{i}}-p_{i},
$$

with respect to the module $\mathrm{M}:=\mathbb{I}_{L}(F) \subset \mathrm{R}^{m}$ we need to consider the morphisms

$$
\begin{aligned}
\mathfrak{s}_{L}: \mathrm{S}^{u} \rightarrow \mathrm{S}^{m} & : \quad \mathfrak{s}_{L}\left(\sum_{i=1}^{u} h_{i} e_{i}\right):=\sum_{i=1}^{u} h_{i} * \mathbf{M}\left(g_{i}\right), \\
\mathfrak{S}_{L}: \mathrm{R}^{u} \rightarrow \mathrm{M} \subset \mathrm{R}^{m} & : \quad{ }{ }_{L}\left(\sum_{i=1}^{u} h_{i} e_{i}\right):=\sum_{i=1}^{u} h_{i} \star g_{i},
\end{aligned}
$$

where the symbols $\left\{e_{1}, \ldots, e_{u}\right\}$ denote the common canonical basis of $\mathrm{S}^{u}$ and $\mathrm{R}^{u}$ which, as $R$-modules, coincide.

We can then consider

- the $\mathcal{T}^{(m)}$-valuation $v: \mathrm{R}^{u} \rightarrow \mathcal{T}^{(m)}$ defined, for each $\sigma:=\sum_{i=1}^{u} h_{i} e_{i} \in \mathrm{R}^{u} \backslash\{0\}$, by

$$
v(\sigma):=\max _{<}\left\{\mathbf{T}\left(h_{i} \star g_{i}\right)\right\}=\max _{<}\left\{\mathbf{T}_{<}\left(h_{i}\right) \circ \mathbf{T}_{<}\left(g_{i}\right)\right\}=\max _{<}\left\{\mathbf{T}_{<}\left(h_{i}\right) \circ \tau_{i} \mathbf{e}_{l_{i}}\right\}=: \delta \epsilon
$$

under which we further have $\mathrm{S}^{u}=G\left(\mathrm{R}^{u}\right)$;

- the corresponding leading form $\mathcal{L}_{L}(\sigma):=\sum_{h \in H} \mathbf{M}\left(h_{h}\right) e_{h} \in \mathrm{S}^{u}$ - which is $\mathcal{T}^{(m)}{ }_{-}$ homogeneous of $\mathcal{T}^{(m)}$-degree $v(\sigma)=\delta \epsilon-$ where

$$
H:=\left\{j: \mathbf{T}_{<}\left(h_{j} \star g_{j}\right)=\mathbf{T}_{<}\left(h_{j}\right) \circ \tau_{j} \mathbf{e}_{l_{j}}=\delta \epsilon=v(\sigma)\right\} .
$$


Figure 2: Canonical Form Algorithms

$\left(g, \sum_{i=1}^{\mu} a_{i} \lambda_{i} \star g_{i}\right):=$ LeftCanonicalForm $(f, F)$

where

$\mathrm{R}:=R[\mathcal{T}], R$ a ring with canonical representatives,

$f \in \mathrm{R}^{m}, F$ is the left Gröbner basis of the left module $\mathrm{I} \subset \mathrm{R}^{m}$

$g:=\operatorname{Can}(f, \mathrm{I}) \in \boldsymbol{\operatorname { R e p }}(\mathrm{I}), a_{i} \in R \backslash\{0\}, \lambda_{i} \in \mathcal{T}, g_{i} \in F$,

$f-g=\sum_{i=1}^{\mu} a_{i} \lambda_{i} \star g_{i}$ is a left weak Gröbner representation in terms of $F$,

$h:=f, \mu:=0, g:=0$

While $h \neq 0$ do

Let $c \tau:=\mathbf{M}(h), \gamma:=\operatorname{Rep}\left(c, \mathrm{I}_{\tau}\right)$

$h:=h-\gamma \tau, g:=g+\gamma \tau$,

If $c \neq \gamma$, let $g_{i} \in F, \lambda_{i} \in \mathcal{T}, a_{i} \in R \backslash\{0\}$ :

$c-\gamma=\sum_{i=\mu+1}^{v} a_{i} \alpha_{\lambda_{i}}\left(\operatorname{lc}\left(g_{i}\right)\right), \mathbf{T}(g)=\lambda_{i} \circ \mathbf{T}\left(g_{i}\right), \mu<i \leq v$,

$h:=h-\sum_{i=\mu+1}^{v} a_{i} \lambda_{i} \star g_{i}, \mu:=v$

$\left(g, \sum_{i=1}^{\mu} g_{i} \star b_{i} \rho_{i}\right):=$ RightCanonicalForm $(f, F)$

where

$\mathrm{R}:=R[\mathcal{T}], R$ a ring with canonical representatives,

$f \in \mathrm{R}^{m}, F$ is the right Gröbner basis of the right module $\mathrm{I} \subset \mathrm{R}^{m}$,

$g:=\operatorname{Can}(f, \mathrm{I}) \in \boldsymbol{\operatorname { R e p }}(\mathrm{I}), b_{i} \in R \backslash\{0\}, \rho_{i} \in \mathcal{T}, g_{i} \in F$,

$f-g=\sum_{i=1}^{\mu} g_{i} \star b_{i} \rho_{i}$ is a right weak Gröbner representation in terms of $F$,

$h:=f, \mu:=0, g:=0$

While $h \neq 0$ do

Let $c \tau:=\mathbf{M}(h), \gamma:=\operatorname{Rep}\left(c, \mathrm{I}_{\tau}\right)$

$h:=h-\gamma \tau, g:=g+\gamma \tau$,

If $c \neq \gamma$, let $g_{i} \in F, \rho_{i} \in \mathcal{T}, b_{i} \in R \backslash\{0\}$ :

$c-\gamma=\sum_{i=\mu+1}^{v} \operatorname{lc}\left(g_{i}\right) b_{i}, \mathbf{T}(g)=\mathbf{T}\left(g_{i}\right) \circ \rho_{i}, \mu<i \leq v$,

$h:=h-\sum_{i=\mu+1}^{v} g_{i} \star \alpha_{\mathbf{T}\left(g_{i}\right)}^{-1}\left(b_{i}\right) \rho_{i}, \mu:=v$, 
Figure 2 (cont.): Canonical Form Algorithms

$\left(g, \sum_{i=1}^{\mu} a_{i} \lambda_{i} \star g_{i} \star \rho_{i}\right):=$ BilateralCanonicalForm $(f, F)$

where

$\mathrm{R}:=R[\mathcal{T}], R$ a ring with canonical representatives,

$f \in \mathrm{R}^{m}, F$ is the bilateral Gröbner basis of the bilateral module $\mathbf{I} \subset \mathbf{R}^{m}$,

$g:=\operatorname{Can}(f, \mathrm{I}) \in \boldsymbol{\operatorname { R e p }}(\mathrm{I}), a_{i} \in R \backslash\{0\}, \lambda_{i}, \rho_{i} \in \mathcal{T}, g_{i} \in F$,

$f-g=\sum_{i=1}^{\mu} a_{i} \lambda_{i} \star g_{i} \star \rho_{i}$ is a bilateral Gröbner representation in terms of $F$,

$h:=f, \mu:=0, g:=0$

While $h \neq 0$ do

Let $c \tau:=\mathbf{M}(h), \gamma:=\operatorname{Rep}\left(c, \mathrm{I}_{\tau}\right)$

$h:=h-\gamma \tau, g:=g+\gamma \tau$,

If $c \neq \gamma$,

let $g_{i} \in F, \lambda_{i}, \rho_{i} \in \mathcal{T}, a_{i} \in R \backslash\{0\}$ :

$c-\gamma=\sum_{i=\mu+1}^{v} a_{i} \alpha_{\lambda_{i}}\left(\operatorname{lc}\left(g_{i}\right)\right), \mathbf{T}(g)=\lambda_{i} \circ \mathbf{T}\left(g_{i}\right) \circ \rho_{i}, \mu<i \leq \nu$,

$h:=h-\sum_{i=\mu+1}^{v} a_{i} \lambda_{i} \star g_{i} \star \rho_{i}, \mu:=v$,

Definition 31. With the notation above and denoting for each set $S \subset \mathrm{R}^{u}, \mathcal{L}_{L}\{S\}:=$ $\left\{\mathcal{L}_{L}(g): g \in S\right\} \subset \mathrm{S}^{u}$,

- for a left R-module $\mathrm{N} \subset \mathrm{R}^{u}$, a set $B \subset \mathrm{N}$ is called a left standard basis if $\mathbb{I}_{L}\left(\mathcal{L}_{L}\{B\}\right)=\mathbb{I}_{L}\left(\mathcal{L}_{L}\{\mathrm{~N}\}\right)$

- for each $h \in \mathrm{N}$ a representation

$$
h=\sum_{i} h_{i} \star g_{i}: h_{i} \in \mathrm{R}, g_{i} \in B,
$$

is called a left standard representation in $\mathrm{R}$ in terms of $B$ iff

$$
v(h) \geq v\left(h_{i} \star g_{i}\right), \text { for each } i \text {; }
$$

- if $u \in \operatorname{ker}\left(\mathfrak{s}_{L}\right)$ is $\mathcal{T}^{(m)}$-homogeneous and $U \in \operatorname{ker}\left(\Im_{L}\right)$ is such that $u=\mathcal{L}_{L}(U)$, we say that $u$ lifts to $U$, or $U$ is a lifting of $u$, or simply $u$ has a lifting;

- a left Gebauer-Möller set for $F$ is any $\mathcal{T}^{(m)}$-homogeneous basis of $\operatorname{ker}\left(\mathfrak{s}_{L}\right)$;

- for each $\mathcal{T}^{(m)}$-homogeneous element $\sigma \in \mathrm{R}^{u}$, we say that $\mathfrak{S}_{L}(\sigma)$ has a left quasiGröbner representation in terms of $F$ if it can be written as $\subseteq_{L}(\sigma)=\sum_{i=1}^{u} l_{i} \star g_{i}$ with

$v(\sigma)>\mathbf{T}\left(l_{i} \star g_{i}\right)=\mathbf{T}\left(l_{i}\right) \circ \mathbf{T}\left(g_{i}\right)$ for each $i$. 
Remark 32. Note that each Gröbner representation of $\mathfrak{\subseteq}_{L}(\sigma)$ in terms of $F$ gives also a quasi-Gröbner representation since $\mathbf{T}\left(l_{i}\right) \circ \mathbf{T}\left(g_{i}\right) \leq \mathbf{T}\left(\Im_{L}(\sigma)\right)<v(\sigma)$; on the other side, a quasi-Gröbner representation grants only $\mathbf{T}\left(l_{i}\right) \circ \mathbf{T}\left(g_{i}\right)<v(\sigma)$ but not necessarily $\mathbf{T}\left(l_{i}\right) \circ \mathbf{T}\left(g_{i}\right) \leq \mathbf{T}\left(\Im_{L}(\sigma)\right)$, since in principle we could have $\mathbf{T}\left(\Im_{L}(\sigma)\right)<\mathbf{T}\left(l_{i}\right) \circ \mathbf{T}\left(g_{i}\right)<$ $v(\sigma)$ so that we don't necessarily obtain a Gröbner representation of the S-polynomial $\Im_{L}(\sigma)$.

This relaxation was introduced by Gebauer-Möller in their reformulation of Buchberger Theory for polynomial rings over a field [18]; in that setting, it allowed to better remove useless S-pairs and thus granted a more efficient reformulation of the algorithm; in the more general setting we are considering now, viz polynomials over rings, it becomes essential also for a smooth reformulation of the theory.

Observe that if $\sigma:=\sum_{j=1}^{u} h_{j} e_{j} \in \operatorname{ker}\left(\Im_{L}\right)$ then denoting

$$
\delta \epsilon:=v(\sigma) \text { and } H:=\left\{j, 1 \leq j \leq u: \mathbf{T}\left(h_{j}\right) \circ \mathbf{T}\left(g_{j}\right)=\delta \epsilon\right\},
$$

its leading form $\mathcal{L}_{L}(\sigma):=\sum_{j=1}^{u} d_{j} \lambda_{j} e_{j} \in \mathrm{S}^{u}$ is $\mathcal{T}^{(m)}$-homogeneous of $\mathcal{T}^{(m)}$-degree $v(\sigma):=\delta \epsilon \in \mathcal{T}^{(m)}$, satisfies

$-0 \neq d_{j} \Longleftrightarrow j \in H$ and $\mathbf{M}\left(h_{j}\right)=d_{j} \lambda_{j}$,

$-\sum_{j=1}^{u} d_{j} \lambda_{j} * \mathbf{M}\left(g_{j}\right)=\sum_{j \in H}\left(d_{j} \lambda_{j}\right) *\left(c_{j} \tau_{j} \mathbf{e}_{l_{j}}\right)=\left(\sum_{j \in H}\left(d_{j} \alpha_{\lambda_{j}}\left(c_{j}\right)\right) \cdot\left(\lambda_{j} \tau_{j}\right)\right) \epsilon=0$,

- $\sum_{j \in H} d_{j} \alpha_{\lambda_{j}}\left(\operatorname{lc}\left(g_{j}\right)\right)=0$ and $\lambda_{j} \circ \mathbf{T}\left(g_{j}\right)=\delta \epsilon$ for each $j \in H$, so that in particular

$-\epsilon=\mathbf{e}_{l_{j}}$ for each $j \in H$,

and belongs to $\operatorname{ker}\left(\mathfrak{s}_{L}\right)$.

Theorem 33 (Möller; Left Lifting Theorem). [34]

With the present notation and denoting $15 \mathfrak{M}(F)$ any left Gebauer-Möller set for $F$, the following conditions are equivalent:

1. F is a left Gröbner basis of $\mathrm{M}$;

2. $f \in \mathrm{M} \Longleftrightarrow f$ has a left Gröbner representation in terms of $F$;

3. for each $\sigma \in\left(\mathfrak{5 M}(F)\right.$, the $S$-polynomial $\Xi_{L}(\sigma)$ has a quasi-Gröbner representation

$$
\Xi_{L}(\sigma)=\sum_{i=1}^{u} l_{i} g_{i}
$$

4. each $\sigma \in(5 \mathfrak{M}(F)$ has a lifting $\operatorname{lift}(\sigma)$;

5. each $\mathcal{T}^{(m)}$-homogeneous element $u \in \operatorname{ker}\left(\mathfrak{s}_{L}\right)$ has a lifting $\operatorname{lift}(u)$.

Proof. 
(1) $\Longrightarrow$ (2) Let $f \in \mathrm{M}$; by assumption

$$
\mathbf{M}(f)=\sum_{i=1}^{u} a_{i} \lambda_{i} * \mathbf{M}\left(g_{i}\right)
$$

where $\left(a_{1} \lambda_{1}, \ldots, a_{u} \lambda_{u}\right)$ is $\mathcal{T}^{(m)}$-homogeneous of $\mathcal{T}^{(m)}$-degree $\mathbf{T}(f)$.

Therefore $g:=f-\sum_{i=1}^{u} a_{i} \lambda_{i} \star g_{i} \in \mathbf{M}$ and $\mathbf{T}(g)<\mathbf{T}(f)$.

Thus, we can assume by induction the existence of a Gröbner representation $g:=\sum_{i=1}^{u} l_{i} \star g_{i}$ of $g$; whence $f:=\sum_{i=1}^{u}\left(a_{i} \lambda_{i}+l_{i}\right) \star g_{i}$ is the required Gröbner representation of $f$.

(2) $\Longrightarrow(3) \Im_{L}(\sigma) \in \mathrm{M}$ and $\mathbf{T}\left(\subseteq_{L}(\sigma)\right)<v(\sigma)$.

(3) $\Longrightarrow$ (4) Let $\varsigma_{L}(\sigma)=\sum_{i=1}^{u} l_{i} \star g_{i}$ be a quasi-Gröbner representation in terms of $F$; then $\mathbf{T}\left(l_{i} \star g_{i}\right)<v(\sigma)$ so that $\operatorname{lift}(\sigma):=\sigma-\sum_{i=1}^{u} l_{i} e_{i}$ is the required lifing of $\sigma$.

(4) $\Longrightarrow$ (5) Let $u:=\sum_{i=1}^{u} a_{i} \lambda_{i} e_{i}, a_{i} \neq 0 \Longrightarrow \lambda_{i} \circ \tau_{i} \mathbf{e}_{i}=v(u)$, be a $\mathcal{T}^{(m)}$-homogeneous element in $\operatorname{ker}\left(\mathfrak{s}_{L}\right)$ of $\mathcal{T}^{(m)}$-degree $v(u)$.

Then there are $c_{\sigma} \in R, \lambda_{\sigma} \in \mathcal{T}$ for which

$$
u=\sum_{\sigma \in(\mathfrak{G M}(F)} c_{\sigma} \lambda_{\sigma} * \sigma, \quad \lambda_{\sigma} \circ v(\sigma)=v(u) .
$$

For each $\sigma \in \mathfrak{G M M}(F)$ denote

$$
\bar{\sigma}:=\sigma-\operatorname{lift}(\sigma)=\mathcal{L}_{L}(\operatorname{lift}(\sigma))-\operatorname{lift}(\sigma):=\sum_{i=1}^{u} l_{i \sigma} e_{i}
$$

and remark that $\mathbf{T}\left(l_{i \sigma}\right) \circ \tau_{i} \mathbf{e}_{i} \leq v(\bar{\sigma})<v(\sigma), \Im_{L}(\operatorname{lift}(\sigma))=0$ and $\varsigma_{L}(\bar{\sigma})=\Im_{L}(\sigma)$. It is sufficient to define

$$
\operatorname{lift}(u):=\sum_{\sigma \in \mathfrak{G M M}(F)} c_{\sigma} \lambda_{\sigma} \star \operatorname{lift}(\sigma) \text {, and } \bar{u}:=\sum_{\sigma \in(\mathfrak{G M}(F)} c_{\sigma} \lambda_{\sigma} \star \bar{\sigma}
$$

to obtain $\operatorname{lift}(u)=u-\bar{u}, \mathcal{L}_{L}(\operatorname{lift}(u))=u, \Xi_{L}(\bar{u})=\Im_{L}(u), \Xi_{L}(\operatorname{lift}(u))=0$.

(5) $\Longrightarrow$ (1) Let $g \in \mathrm{M}$, so that there are $l_{i} \in \mathrm{R}$, such that $\sigma_{1}:=\sum_{i=1}^{u} l_{i} e_{i} \in \mathrm{R}^{u}$ satisfies $g=\widetilde{S}_{L}\left(\sigma_{1}\right)=\sum_{i=1}^{u} l_{i} \star g_{i}$.

Denoting $H:=\left\{i: \mathbf{T}\left(l_{i}\right) \circ \tau_{i} \mathbf{e}_{l_{i}}=v\left(\sigma_{1}\right)\right\}$, then either

- $\mathbf{T}(g)=v\left(\sigma_{1}\right)$, so that $\mathbf{M}(g)=\sum_{i \in H} \mathbf{M}\left(l_{i}\right) * \mathbf{M}\left(g_{i}\right) \in \mathbf{M}\left\{\mathbb{I}_{L}(\mathbf{M}\{F\})\right\}$ and we are through, or

- $\mathbf{T}(g)<v\left(\sigma_{1}\right), 0=\sum_{i \in H} \mathbf{M}\left(l_{i}\right) * \mathbf{M}\left(g_{i}\right)=\mathfrak{s}_{L}\left(\mathcal{L}_{L}\left(\sigma_{1}\right)\right)$ and the $\mathcal{T}^{(m)}$-homogeneous element $\mathcal{L}_{L}\left(\sigma_{1}\right) \in \operatorname{ker}\left(\mathfrak{s}_{L}\right)$ has a lifting $U:=\mathcal{L}_{L}\left(\sigma_{1}\right)-\sum_{i=1}^{u} l_{i}^{\prime} e_{i}$ with

$$
\sum_{i=1}^{u} l_{i}^{\prime} \star g_{i}=\sum_{i \in H} \mathbf{M}\left(l_{i}\right) \star g_{i} \text { and } \mathbf{T}\left(l_{i}^{\prime}\right) \circ \tau_{i} \mathbf{e}_{i}<v\left(\sigma_{1}\right),
$$


so that $g=\mathfrak{S}_{L}\left(\sigma_{2}\right)$ and $v\left(\sigma_{2}\right)<v\left(\sigma_{1}\right)$ for

$$
\sigma_{2}:=\sum_{i \notin H} l_{i} e_{i}+\sum_{i \in H}\left(l_{i}-\mathbf{M}\left(l_{i}\right)\right) e_{i}+\sum_{i=1}^{u} l_{i}^{\prime} e_{i} \in \mathrm{R}^{u}
$$

and the claim follows by the well-orderedness of $<$.

Theorem 34 (Janet-Schreyer). [19, 46, 47]

With the same notation the equivalent conditions (1-5) imply that

6. $\left\{\operatorname{lift}(\sigma): \sigma \in(5 \mathfrak{M}(F)\}\right.$ is a left standard basis of $\operatorname{ker}\left(\Im_{L}\right)$.

Proof. (4) $\Longrightarrow$ (6) Let $\sigma_{1}:=\sum_{i=1}^{u} l_{i} e_{i} \in \operatorname{ker}\left(\Im_{L}\right) \subset \mathrm{R}^{u}$.

Denoting $H:=\left\{i: \mathbf{T}\left(l_{i}\right) \circ \tau_{i} \mathbf{e}_{l_{i}}=v\left(\sigma_{1}\right)\right\}$, we have

$$
\mathcal{L}_{L}\left(\sigma_{1}\right)=\sum_{i \in H} \mathbf{M}\left(l_{i}\right) e_{i} \in \operatorname{ker}\left(\mathfrak{s}_{L}\right)
$$

and there is a $\mathcal{T}^{(m)}$-homogeneous representation

$$
\mathcal{L}_{L}\left(\sigma_{1}\right)=\sum_{\sigma \in \mathfrak{G M}(F)} c_{\sigma} \lambda_{\sigma} * \sigma, \lambda_{\sigma} \circ v(\sigma)=v\left(\sigma_{1}\right), c_{\sigma} \in R, \lambda_{\sigma} \in \mathcal{T}
$$

Then

$$
\begin{aligned}
& \sigma_{2}:=\sigma_{1}-\sum_{\sigma \in \mathfrak{G M M}(F)} c_{\sigma} \lambda_{\sigma} \star \operatorname{lift}(\sigma) \\
& =\sigma_{1}-\sum_{\sigma \in \in \mathfrak{G M}(F)} c_{\sigma} \lambda_{\sigma} \star(\sigma-\bar{\sigma}) \\
& =\left(\sigma_{1}-\mathcal{L}_{L}\left(\sigma_{1}\right)\right)+\sum_{\sigma \in \in \mathfrak{G M}(F)} c_{\sigma} \lambda_{\sigma} \star \bar{\sigma} \\
& =\sum_{i \in H}\left(\left(l_{i}-\mathbf{M}\left(l_{i}\right)\right)+\sum_{\sigma \in \mathfrak{G M M}(F)} \lambda_{\sigma} \star l_{i \sigma}\right) e_{i}+\sum_{i \notin H}\left(l_{i}+\sum_{\sigma \in \mathfrak{G} \mathfrak{M}(F)} \lambda_{\sigma} \star l_{i \sigma}\right) e_{i}
\end{aligned}
$$

satisfies both $\sigma_{2} \in \operatorname{ker}\left(\Xi_{L}\right)$ and $v\left(\sigma_{2}\right)<v\left(\sigma_{1}\right)$; thus the claim follows by induction.

Example 35. Let us consider the ring of Example 17 and three elements $f_{1}, f_{2}, f_{3} \in \mathbf{R}$ with

$$
\mathbf{M}\left(f_{1}\right)=(5 x-1) Y_{1} Y_{2}^{2} Y_{3}^{2}, \mathbf{M}\left(f_{2}\right)=(5 x-1) Y_{1}^{2} Y_{2} Y_{3}^{2}, \mathbf{M}\left(f_{3}\right)=(5 x-1) Y_{1}^{2} Y_{2}^{2} Y_{3}
$$

Under the natural $\mathcal{T}$-pseudovaluation on $\mathrm{R}^{3}$, an element

$$
\sigma:=\left(\alpha Y_{1}^{\alpha_{1}} Y_{2}^{\alpha_{2}} Y_{3}^{\alpha_{3}}, \beta Y_{1}^{\beta_{1}} Y_{2}^{\beta_{2}} Y_{3}^{\beta_{3}}, \gamma Y_{1}^{\gamma_{1}} Y_{2}^{\gamma_{2}} Y_{3}^{\gamma_{3}}\right) \in \mathrm{S}^{3}
$$


is homogeneous of $\mathcal{T}$-degree $Y_{1}^{a+2} Y_{2}^{b+2} Y_{3}^{c+2}$ iff

$$
\alpha_{1}-1=\beta_{1}=\gamma_{1}=: a, \alpha_{2}=\beta_{2}-1=\gamma_{2}=: b, \alpha_{3}=\beta_{3}=\gamma_{3}-1=: c \text {. }
$$

Let us now specialize ourselves to the case $a=b=c=0$ and consider the $\mathbb{Z}$ module of the homogeneous syzygies of $\mathcal{T}$-degree $Y_{1}^{2} Y_{2}^{2} Y_{3}^{2} ; 9$ is a syzygy in $\operatorname{ker}\left(\mathfrak{s}_{L}\right)$ iff

$$
\begin{aligned}
0 & =\mathfrak{s}_{L}(\sigma) \\
& =\alpha Y_{1} * \mathbf{M}\left(f_{1}\right)+\beta Y_{2} * \mathbf{M}\left(f_{2}\right)+\gamma Y_{3} * \mathbf{M}\left(f_{3}\right) \\
& =\left(\alpha\left(y^{2}-1\right)+\beta\left(y^{3}-1\right)+\gamma\left(y^{4}-1\right)\right) Y_{1}^{2} Y_{2}^{2} Y_{3}^{2} .
\end{aligned}
$$

A minimal Gebauer-Möller set consists of

$$
\sigma_{1}:=\left(-\left(y^{2}+y+1\right) Y_{1},(y+1) Y_{2}, 0\right) \text { and } \sigma_{2}:=\left(-\left(y^{2}+1\right) Y_{1}, 0, Y_{3}\right) .
$$

In fact a generic syzygy (9) satisfies

$$
\alpha(y+1)+\beta\left(y^{2}+y+1\right)+\gamma\left(y^{2}+1\right)(y+1)=0
$$

so that $(y+1) \mid \beta$ and setting $\beta=(y+1) \delta$ we have $\alpha=-\delta\left(y^{2}+y+1\right)-\gamma\left(y^{2}+1\right)$ whence

$$
\sigma:=\left(\left(-\delta\left(y^{2}+y+1\right)-\gamma\left(y^{2}+1\right)\right) Y_{1},(y+1) \delta Y_{2}, \gamma Y_{3}\right)=\delta \sigma_{1}+\gamma \sigma_{2} .
$$

Remark 36. We can consider also the homogeneous syzygy of $\mathcal{T}$-degree $Y_{1}^{2} Y_{2}^{2} Y_{3}^{2}$

$$
\sigma_{3}:=\left(0,-\left(y^{2}+1\right)(y+1) Y_{2},\left(y^{2}+y+1\right) Y_{3}\right)-\left(y^{2}+1\right) \sigma_{1}+\left(y^{2}+y+1\right) \sigma_{2} .
$$

Moreover, since

$$
1=\left(y^{2}+y+1\right)-y(y+1)=\left(y^{3}+y^{2}+y+1\right)-y\left(y^{2}+y+1\right)
$$

setting

$$
\varsigma_{A}:=\left(y Y_{1}, Y_{2}, 0\right) \in \mathrm{S}^{3}, \varsigma_{B}:=\left(0,-y Y_{2}, Y_{3}\right) \in \mathrm{S}^{3}
$$

we have

$$
\mathfrak{s}_{L}\left(\varsigma_{A}\right)=\mathfrak{s}_{L}\left(\varsigma_{B}\right)=(y-1) Y_{1}^{2} Y_{2}^{2} Y_{3}^{2}
$$

note that

$$
\varsigma_{A}-\varsigma_{B}:=\left(y Y_{1},(y+1) Y_{2}, Y_{3}\right)=\sigma_{1}+\sigma_{2} \in \operatorname{ker}\left(\mathfrak{s}_{L}\right) .
$$

Example 35 (cont.). Setting now $\tau:=Y_{1}^{a} Y_{2}^{b} Y_{3}^{c}$ and $z:=y^{2^{a} 3^{b} 4^{c}}$, for the syzygy (9) we have

$$
\begin{aligned}
0 & =\mathfrak{s}_{L}(\sigma) \\
& =\alpha \tau Y_{1} * \mathbf{M}\left(f_{1}\right)+\beta \tau Y_{2} * \mathbf{M}\left(f_{2}\right)+\gamma \tau Y_{3} * \mathbf{M}\left(f_{3}\right) \\
& =\left(\alpha \tau *\left(y^{2}-1\right)+\beta \tau *\left(y^{3}-1\right)+\gamma \tau *\left(y^{4}-1\right)\right) Y_{1}^{2} Y_{2}^{2} Y_{3}^{2} . \\
& =\left(\alpha\left(z^{2}-1\right)+\beta\left(z^{3}-1\right)+\gamma\left(z^{4}-1\right)\right) Y_{1}^{2} Y_{2}^{2} Y_{3}^{2} \tau
\end{aligned}
$$


whence

$$
\alpha=-\delta\left(z^{2}+z+1\right)-\gamma\left(z^{2}+1\right), \quad \beta=(y+1) \delta
$$

and

$$
\sigma:=\beta \tau * \sigma_{1}+\gamma \tau * \sigma_{2}
$$

Thus, $\left\{\sigma_{1}, \sigma_{2}\right\}$ is a minimal basis of $\operatorname{ker}\left(\mathfrak{s}_{L}\right)$.

\subsection{Bilateral case}

Considering $\mathrm{R}$ as a left $R$-module, the adaptation of Möller lifting theorem to the bilateral case requires a few elementary adaptations; given a finite set

$$
F:=\left\{g_{1}, \ldots, g_{u}\right\} \subset \mathrm{R}^{m}, g_{i}=\mathbf{M}\left(g_{i}\right)-p_{i}=: c_{i} \tau_{i} \mathbf{e}_{\iota_{i}}-p_{i}
$$

and the bilateral module $\mathrm{M}:=\mathbb{I}_{2}(F)$, denote

$$
\hat{R}:=\{a \in R: a h=a \star h=h \star a, \text { for each } h \in \mathrm{R}\}
$$

the commutative subring $\hat{R} \subset R$ of $R$ consisting of the elements belonging to the center of $\mathrm{R}$ and remark that the subring of $R$ generated by $\mathbf{1}_{R}$ is a subring of $\hat{R}$ and that $\hat{R}$ is also a subring of the center of the associated graded Ore extension $S$ of $R$.

Considering both the R-bimodule $\mathrm{R} \otimes_{\hat{R}} \mathrm{R}^{\mathrm{op}}$ and the $\mathrm{S}$-bimodule $\mathrm{S} \otimes_{\hat{R}} \mathrm{~S}^{\mathrm{op}}$, which, as sets, coincide, we impose on the bilateral $R$-module $\left(R \otimes_{\hat{R}} R^{\text {op }}\right)^{u}$, whose canonical basis is denoted $\left\{e_{1}, \ldots, e_{u}\right\}$ and whose generic element has the shape

$$
\sum_{i} a_{i} \lambda_{i} e_{\ell_{i}} b_{i} \rho_{i}, a_{i}, b_{i} \in R \backslash\{0\}, \lambda_{i}, \rho_{i} \in \mathcal{T}, 1 \leq \ell_{i} \leq u
$$

the $\mathcal{T}^{(m)}$-graded structure given by the valuation $v:\left(\mathrm{R} \otimes_{\hat{R}} \mathrm{R}^{\mathrm{op}}\right)^{u} \rightarrow \mathcal{T}$ as

$$
v(\sigma):=\max _{<}\left\{\mathbf{T}\left(\lambda_{i} \star g_{\ell_{i}} \star \rho_{i}\right)\right\}=\max _{<}\left\{\lambda_{i} * \mathbf{T}\left(g_{\ell_{i}}\right) * \rho_{i}\right\}=\max _{<}\left\{\lambda_{i} \circ \tau_{\ell_{i}} \circ \rho_{i} \mathbf{e}_{l_{i}}\right\}=: \delta \epsilon
$$

for each

$$
\sigma:=\sum_{i} a_{i} \lambda_{i} e_{\ell_{i}} b_{i} \rho_{i} \in\left(\mathrm{R} \otimes_{\hat{R}} \mathrm{R}^{\mathrm{op}}\right)^{u} \backslash\{0\}
$$

so that

$$
G\left(\left(\mathrm{R} \otimes_{\hat{R}} \mathrm{R}^{\mathrm{op}}\right)^{u}\right)=\left(G\left(\mathrm{R} \otimes_{\hat{R}} \mathrm{R}^{\mathrm{op}}\right)\right)^{u}=\left(\mathrm{S} \otimes_{\hat{R}} \mathrm{~S}^{\mathrm{op}}\right)^{u}
$$

and its corresponding $\mathcal{T}^{(m)}$-homogeneous leading form is

$$
\mathcal{L}_{2}(\sigma):=\sum_{h \in H} a_{h} \lambda_{h} e_{\ell_{h}} b_{h} \rho_{h} \in\left(\mathrm{S} \otimes_{\hat{R}} \mathrm{~S}^{\mathrm{op}}\right)^{u}
$$

where $H:=\left\{j: \lambda_{j} \circ \tau_{j} \circ \rho_{j} \mathbf{e}_{\iota_{\ell_{j}}}=v(\sigma)=\delta \epsilon\right\}$; we also denote, for each set $S \subset$ $\left(\mathrm{R} \otimes_{\hat{R}} \mathrm{R}^{\mathrm{op}}\right)^{u}$,

$$
\mathcal{L}_{2}\{S\}:=\left\{\mathcal{L}_{2}(g): g \in S\right\} \subset\left(\mathrm{S} \otimes_{\hat{R}} \mathrm{~S}^{\mathrm{op}}\right)^{u}
$$


We can therefore consider the morphisms

$$
\begin{gathered}
\mathfrak{s}_{2}:\left(\mathrm{S} \otimes_{\hat{R}} \mathrm{~S}^{\mathrm{op}}\right)^{u} \rightarrow \mathrm{S}^{m} \quad: \quad \mathfrak{s}_{2}\left(\sum_{i} a_{i} \lambda_{i} e_{\ell_{i}} b_{i} \rho_{i}\right):=\sum_{i} a_{i} \lambda_{i} * \mathbf{M}\left(g_{\ell_{i}}\right) * b_{i} \rho_{i}, \\
\mathfrak{S}_{2}:\left(\mathrm{R} \otimes_{\hat{R}} \mathrm{R}^{\mathrm{op}}\right)^{u} \rightarrow \mathrm{R}^{m}: \mathbb{S}_{2}\left(\sum_{i} a_{i} \lambda_{i} e_{\ell_{i}} b_{i} \rho_{i}\right):=\sum_{i} a_{i} \lambda_{i} \star g_{\ell_{i}} \star b_{i} \rho_{i} .
\end{gathered}
$$

Definition 36. With the notation above

- for a bilateral R-module $\mathrm{N}$, a set $F \subset \mathrm{N}$ is called a bilateral standard basis if

$$
\mathbb{I}_{2}\left(\mathcal{L}_{2}\{F\}\right)=\mathbb{I}_{2}\left(\mathcal{L}_{2}\{\mathrm{~N}\}\right)
$$

- for each $h \in \mathrm{N}$ a representation

$$
h=\sum_{i} a_{i} \lambda_{i} \star g_{\ell_{i}} \star b_{i} \rho_{i}: a_{i}, b_{i} \in R \backslash\{0\}, \lambda_{i}, \rho_{i} \in \mathcal{T}, g_{\ell_{i}} \in F,
$$

is called a standard representation in $\mathrm{R}$ in terms of $F$ iff

$$
v(h) \geq v\left(\lambda_{i} \star g_{\ell_{i}} \star \rho_{i}\right)=\lambda_{i} \circ v\left(g_{\ell_{i}}\right) \circ \rho_{i}, \text { for each } i ;
$$

- if $u \in \operatorname{ker}\left(\mathfrak{s}_{2}\right)$ is $\mathcal{T}^{(m)}$-homogeneous and $U \in \operatorname{ker}\left(\mathbb{S}_{2}\right)$ is such that $u=\mathcal{L}_{2}(U)$, we say that $u$ lifts to $U$, or $U$ is a lifting of $u$, or simply $u$ has a lifting;

- a bilateral Gebauer-Möller set for $F$ is any $\mathcal{T}^{(m)}$-homogeneous basis of $\operatorname{ker}\left(\mathfrak{s}_{2}\right)$;

- for each $\mathcal{T}^{(m)}$-homogeneous element $\sigma \in\left(\mathrm{R} \otimes_{\hat{R}} \mathrm{R}^{\mathrm{op}}\right)^{u}$, we say that $\mathfrak{S}_{2}(\sigma)$ has a bilateral quasi-Gröbner representation in terms of $G$ if it can be written as

$$
\widetilde{\Xi}_{2}(\sigma)=\sum_{i} a_{i} \lambda_{i} \star g_{\ell_{i}} \star b_{i} \rho_{i}: a_{i}, b_{i} \in R \backslash\{0\}, \lambda_{i}, \rho_{i} \in \mathcal{T}, g_{\ell_{i}} \in F
$$

with $\lambda_{i} \circ \mathbf{T}\left(g_{\ell_{i}}\right) \circ \rho_{i}<v(\sigma)$ for each $i$.

Theorem 37 (Möller-Pritchard). With the present notation and denoting $(5 \mathfrak{M}(F)$ any bilateral Gebauer-Möller set for $F$, the following conditions are equivalent:

1. F is a bilateral Gröbner basis of $\mathrm{M}$;

2. $f \in \mathrm{M} \Longleftrightarrow f$ has a bilateral Gröbner representation in terms of $F$;

3. for each $\sigma \in\left(\mathfrak{F M}(F)\right.$, the bilateral $S$-polynomial $\mathfrak{\Xi}_{2}(\sigma)$ has a bilateral quasiGröbner representation $\Xi_{2}(\sigma)=\sum_{l=1}^{\mu} a_{l} \lambda_{l} \star g_{\ell_{l}} \star b_{l} \rho_{l}$, in terms of $F$;

4. each $\sigma \in(\mathfrak{F M}(F)$ has a lifting $\operatorname{lift}(\sigma)$;

5. each $\mathcal{T}^{(m)}$-homogeneous element $u \in \operatorname{ker}\left(\mathfrak{s}_{2}\right)$ has a lifting $\operatorname{lift}(u)$.

Proof. 
(1) $\Longrightarrow$ (2) Let $f \in \mathrm{M}$; by assumption

$$
\mathbf{M}(f)=\sum_{i=1}^{\mu} a_{i} \lambda_{i} * \mathbf{M}\left(g_{\ell_{i}}\right) * b_{i} \rho_{i}
$$

where $\sum_{i=1}^{\mu} a_{i} \lambda_{i} e_{\ell_{i}} b_{i} \rho_{i} \in\left(\mathrm{S} \otimes_{\hat{R}} \mathrm{~S}^{\mathrm{op}}\right)^{u}$ is $\mathcal{T}^{(m)}$-homogeneous of $\mathcal{T}^{(m)}$-degree $\mathbf{T}(f)$. Therefore $g:=f-\sum_{i=1}^{\mu} a_{i} \lambda_{i} \star g_{\ell_{i}} \star b_{i} \rho_{i} \in \mathrm{M}$ and $\mathbf{T}(g)<\mathbf{T}(f)$.

Thus, the claim follows by induction since $<$ is a well-ordering.

$(2) \Longrightarrow(3) \mathbb{S}_{2}(\sigma) \in \mathrm{M}$ and $\mathbf{T}\left(\mathfrak{S}_{2}(\sigma)\right)<v(\sigma)$.

(3) $\Longrightarrow$ (4) Let

$$
\widetilde{S}_{2}(\sigma)=\sum_{i=1}^{\mu} a_{i} \lambda_{i} \star g_{\ell_{i}} \star b_{i} \rho_{i}, v(\sigma)>\lambda_{i} \circ \tau_{\ell_{i}} \circ \rho_{i} \mathbf{e}_{t_{\ell_{i}}}
$$

be a bilateral quasi-Gröbner representation in terms of $F$; then

$$
\operatorname{lift}(\sigma):=\sigma-\sum_{i=1}^{\mu} a_{i} \lambda_{i} e_{\ell_{i}} b_{i} \rho_{i}
$$

is the required lifting of $\sigma$.

(4) $\Longrightarrow$ (5) Let $u:=\sum_{i} a_{i} \lambda_{i} e_{\ell_{i}} b_{i} \rho_{i} \in\left(\mathrm{S} \otimes_{\hat{R}} \mathrm{~S}^{\mathrm{op}}\right)^{u}, \lambda_{i} \circ \tau_{\ell_{i}} \circ \rho_{i} \mathbf{e}_{\ell_{\ell_{i}}}=v(u)$, be a $\mathcal{T}^{(m)}{ }_{-}$ homogeneous element in $\operatorname{ker}\left(\mathfrak{s}_{2}\right)$ of $\mathcal{T}^{(m)}$-degree $v(u)$.

Then there are $\lambda_{\sigma}, \rho_{\sigma} \in \mathcal{T}, a_{\sigma}, b_{\sigma} \in R \backslash\{0\}$, for which

$$
u=\sum_{\sigma \in(\mathfrak{G M}(F)} a_{\sigma} \lambda_{\sigma} * \sigma * b_{\sigma} \rho_{\sigma}, \lambda_{\sigma} \circ v(\sigma) \circ \rho_{\sigma}=v(u) .
$$

For each $\sigma \in \mathfrak{b M}(F)$ denote

$$
\bar{\sigma}:=\sigma-\operatorname{lift}(\sigma)=\mathcal{L}_{2}(\operatorname{lift}(\sigma))-\operatorname{lift}(\sigma):=\sum_{i=1}^{\mu_{\sigma}} a_{i \sigma} \lambda_{i \sigma} e_{\ell_{i \sigma}} b_{i \sigma} \rho_{i \sigma} \in\left(\mathrm{R} \otimes_{\hat{R}} \mathrm{R}^{\mathrm{op}}\right)^{u}
$$

and remark that $\lambda_{i \sigma} \circ \tau_{\ell_{i \sigma}} \circ \rho_{i \sigma} \mathbf{e}_{\ell_{i \sigma}} \leq v(\bar{\sigma})<v(\sigma)$ and $\mathfrak{S}_{2}(\bar{\sigma})=\mathbb{S}_{2}(\sigma)$.

It is sufficient to define

$$
\operatorname{lift}(u):=\sum_{\sigma \in(\mathfrak{G M M}(F)} a_{\sigma} \lambda_{\sigma} \star \operatorname{lift}(\sigma) \star b_{\sigma} \rho_{\sigma} \text {, and } \bar{u}:=\sum_{\sigma \in(\mathfrak{G M M}(F)} a_{\sigma} \lambda_{\sigma} \star \bar{\sigma} \star b_{\sigma} \rho_{\sigma}
$$

to obtain

$$
\operatorname{lift}(u)=u-\bar{u}, \mathcal{L}_{2}(\operatorname{lift}(u))=u, \Im_{2}(\bar{u})=\subseteq_{2}(u), \subseteq_{2}(\operatorname{lift}(u))=0 .
$$


(5) $\Longrightarrow$ (1) Let $g \in \mathrm{M}$, so that there are $\lambda_{i}, \rho_{i} \in \mathcal{T}, a_{i}, b_{i} \in R \backslash\{0\}, 1 \leq \ell_{i} \leq u$, such that $\sigma_{1}:=\sum_{i=1}^{\mu} a_{i} \lambda_{i} e_{e_{i}} b_{i} \rho_{i} \in\left(\mathrm{R} \otimes_{\hat{R}} \mathrm{R}^{\text {op }}\right)^{u}$ satisfies

$$
g=\subseteq_{2}\left(\sigma_{1}\right)=\sum_{i=1}^{\mu} a_{i} \lambda_{i} \star g_{\ell_{i}} \star b_{i} \rho_{i} .
$$

Denoting $H:=\left\{i: \lambda_{i} \circ \mathbf{T}\left(g_{\ell_{i}}\right) \circ \rho_{i}=\lambda_{i} \circ \tau_{\ell_{i}} \circ \rho_{i} \mathbf{e}_{\iota_{i}}=v\left(\sigma_{1}\right)\right\}$, then either

- $v\left(\sigma_{1}\right)=\mathbf{T}(g)$ so that, for each $i \in H, \mathbf{M}\left(a_{i} \lambda_{i} \star \mathbf{M}\left(g_{\ell_{i}}\right) \star b_{i} \rho_{i}\right)=a_{i} \lambda_{i} *$ $\mathbf{M}\left(g_{\ell_{i}}\right) * b_{i} \rho_{i}$ and

$$
\mathbf{M}(g)=\sum_{i \in H} a_{i} \lambda_{i} * \mathbf{M}\left(g_{\ell_{i}}\right) * b_{i} \rho_{i} \in \mathbf{M}\left\{\mathbb{I}_{2}(\mathbf{M}\{F\})\right\},
$$

and we are through, or

- $\mathbf{T}(g)<v\left(\sigma_{1}\right)$, in which case $0=\sum_{i \in H} a_{i} \lambda_{i} * \mathbf{M}\left(g_{\ell_{i}}\right) * b_{i} \rho_{i}=\mathfrak{s}_{2}\left(\mathcal{L}_{2}\left(\sigma_{1}\right)\right)$ and the $\mathcal{T}^{(m)}$-homogeneous element $\mathcal{L}_{2}\left(\sigma_{1}\right) \in \operatorname{ker}\left(\mathfrak{s}_{2}\right)$ has a lifting

$$
U:=\mathcal{L}_{2}\left(\sigma_{1}\right)-\sum_{j=1}^{v} a_{j} \lambda_{j} e_{\ell_{j}} b_{j} \rho_{j} \in\left(\mathrm{R} \otimes_{\hat{R}} \mathrm{R}^{\mathrm{op}}\right)^{u}
$$

with

$$
\sum_{j=1}^{v} a_{j} \lambda_{j} \star g_{\ell_{j}} \star b_{j} \rho_{j}=\sum_{i \in H} a_{i} \lambda_{i} \star g_{\ell_{i}} \star b_{i} \rho_{i} \text { and } \lambda_{j} \circ \tau_{l_{j}} \circ \rho_{j} \mathbf{e}_{\iota_{\ell_{j}}}<v\left(\sigma_{1}\right)
$$

so that $g=\mathfrak{\Xi}_{2}\left(\sigma_{2}\right)$ and $v\left(\sigma_{2}\right)<v\left(\sigma_{1}\right)$ holds for

$$
\sigma_{2}:=\sum_{i \notin H} a_{i} \lambda_{i} e_{\ell_{i}} b_{i} \rho_{i}+\sum_{j=1}^{v} a_{j} \lambda_{j} e_{j} b_{j} \rho_{j} \in\left(\mathrm{R} \otimes_{\hat{R}} \mathrm{R}^{\mathrm{op}}\right)^{u}
$$

and the claim follows by the well-orderedness of $<$.

Theorem 38 (Janet-Schreyer).

With the same notation the equivalent conditions (1-5) imply that

6. $\{\operatorname{lift}(\sigma): \sigma \in \mathfrak{5 M}(F)\}$ is a bilateral standard basis of $\operatorname{ker}\left(\widetilde{\Xi}_{2}\right)$.

Proof. (4) $\Longrightarrow$ (6) Let $\sigma_{1}:=\sum_{i=1}^{\mu} a_{i} \lambda_{i} e_{\ell_{i}} b_{i} \rho_{i} \in \operatorname{ker}\left(\Xi_{2}\right) \subset\left(\mathrm{R} \otimes_{\hat{R}} \mathrm{R}^{\text {op }}\right)^{u}$.

Denoting $H:=\left\{i: \lambda_{i} \circ \tau_{\ell_{i}} \circ \rho_{i} \mathbf{e}_{\ell_{\ell_{i}}}=v\left(\sigma_{1}\right)\right\}$, we have

$$
\mathcal{L}_{2}\left(\sigma_{1}\right)=\sum_{i \in H} a_{i} \lambda_{i} e_{\ell_{i}} b_{i} \rho_{i} \in \operatorname{ker}\left(\mathfrak{s}_{2}\right)
$$

and there is a $\mathcal{T}^{(m)}$-homogeneous representation

$$
\mathcal{L}_{2}\left(\sigma_{1}\right)=\sum_{\sigma \in(\mathfrak{G} \mathfrak{M}(F)} a_{\sigma} \lambda_{\sigma} * \sigma * b_{\sigma} \rho_{\sigma}, \lambda_{\sigma} \circ v(\sigma) \circ \rho=v\left(\sigma_{1}\right)
$$


with $\lambda_{\sigma}, \rho_{\sigma} \in \mathcal{T}, a_{\sigma}, b_{\sigma} \in R \backslash\{0\}$.

Then

$$
\begin{aligned}
\sigma_{2} & :=\sigma_{1}-\sum_{\sigma \in \mathfrak{F} \mathfrak{M}(F)} a_{\sigma} \lambda_{\sigma} \star \operatorname{lift}(\sigma) \star b_{\sigma} \rho_{\sigma} \\
& =\sigma_{1}-\sum_{\sigma \in \mathfrak{G} \mathfrak{M}(F)} a_{\sigma} \lambda_{\sigma} \star(\sigma-\bar{\sigma}) \star b_{\sigma} \rho_{\sigma} \\
& =\sigma_{1}-\mathcal{L}_{2}\left(\sigma_{1}\right)+\sum_{\sigma \in \mathfrak{G} \mathfrak{M}(F)} a_{\sigma} \lambda_{\sigma} \star \bar{\sigma} \star b_{\sigma} \rho_{\sigma} \\
& =\sum_{i \notin H} a_{i} \lambda_{i} e_{\ell_{i}} b_{i} \rho_{i} \\
& +\sum_{\sigma \in \mathfrak{G} \mathfrak{M}(F)} \sum_{i=1}^{\mu_{\sigma}}\left(\left(a_{\sigma} \alpha_{\lambda_{\sigma}}\left(a_{i \sigma}\right)\right) \cdot\left(\lambda_{\sigma} \circ \lambda_{i \sigma}\right)\right) e_{\ell_{i \sigma}}\left(\left(b_{i \sigma} \alpha_{\rho_{\sigma}}\left(b_{\sigma}\right)\right) \cdot\left(\rho_{i \sigma} \circ \rho_{\sigma}\right)\right)
\end{aligned}
$$

satisfies both $\sigma_{2} \in \operatorname{ker}\left(\mathcal{S}_{2}\right)$ and $v\left(\sigma_{2}\right)<v\left(\sigma_{1}\right)$; thus the claim follows by induction.

\subsection{Restricted case}

In order to deal with restricted modules, we need simply to adapt and simplify the bilateral case.

Thus, we consider both the left $R$-modules $R \otimes \mathrm{R}^{\mathrm{op}}$ and $R \otimes \mathrm{S}^{\mathrm{op}}$, which, as sets, coincide, we impose on the bilateral $R$-module $\left(R \otimes \mathrm{R}^{\mathrm{op}}\right)^{u}$, whose canonical basis is denoted $\left\{e_{1}, \ldots, e_{u}\right\}$ and whose generic element has the shape

$$
\sum_{i} a_{i} e_{\ell_{i}} \rho_{i}, a_{i} \in R \backslash\{0\}, \rho_{i} \in \mathcal{T}, 1 \leq \ell_{i} \leq u,
$$

the $\mathcal{T}^{(m)}$-graded structure given by the valuation $v:\left(R \otimes \mathrm{R}^{\mathrm{op}}\right)^{u} \rightarrow \mathcal{T}$ as

$$
v(\sigma):=\max _{<}\left\{\mathbf{T}\left(g_{\ell_{i}} \star \rho_{i}\right)\right\}=\max _{<}\left\{\mathbf{T}\left(g_{\ell_{i}}\right) * \rho_{i}\right\}=\max _{<}\left\{\tau_{\ell_{i}} \circ \rho_{i} \mathbf{e}_{t_{i}}\right\}=: \delta \epsilon
$$

for each

$$
\sigma:=\sum_{i} a_{i} e_{\ell_{i}} \rho_{i} \in\left(R \otimes_{\hat{R}} \mathrm{R}^{\mathrm{op}}\right)^{u} \backslash\{0\}
$$

so that

$$
G\left(\left(R \otimes \mathrm{R}^{\mathrm{op}}\right)^{u}\right)=\left(G\left(R \otimes \mathrm{R}^{\mathrm{op}}\right)\right)^{u}=\left(R \otimes \mathrm{S}^{\mathrm{op}}\right)^{u}
$$

and its corresponding $\mathcal{T}^{(m)}$-homogeneous leading form is

$$
\mathcal{L}_{W}(\sigma):=\sum_{h \in H} a_{h} e_{\ell_{h}} \rho_{h} \in\left(R \otimes \mathrm{S}^{\mathrm{op}}\right)^{u}
$$

where $H:=\left\{j: \tau_{j} \circ \rho_{j} \mathbf{e}_{\iota_{\ell_{j}}}=v(\sigma)=\delta \epsilon\right\}$; we also denote, for each set $S \subset\left(R \otimes \mathrm{R}^{\mathrm{op}}\right)^{u}$,

$$
\mathcal{L}_{W}\{S\}:=\left\{\mathcal{L}_{W}(g): g \in S\right\} \subset\left(R \otimes S^{\mathrm{op}}\right)^{u} .
$$


We can therefore consider the morphisms

$$
\begin{gathered}
\mathfrak{s}_{W}:\left(R \otimes \mathrm{S}^{\mathrm{op}}\right)^{u} \rightarrow \mathrm{S}^{m} \quad: \quad \mathfrak{s}_{W}\left(\sum_{i} a_{i} e_{\ell_{i}} \rho_{i}\right):=\sum_{i} a_{i} \mathbf{M}\left(g_{\ell_{i}}\right) * \rho_{i}, \\
\mathfrak{S}_{W}:\left(R \otimes \mathrm{R}^{\mathrm{op}}\right)^{u} \rightarrow \mathrm{R}^{m} \quad: \quad \Im_{W}\left(\sum_{i} a_{i} e_{\ell_{i}} \rho_{i}\right):=\sum_{i} a_{i} g_{\ell_{i}} \star \rho_{i} .
\end{gathered}
$$

Definition 39. With the notation above

- for a restricted module $\mathrm{N}$, a set $F \subset \mathrm{N}$ is called a restricted standard basis if

$$
\mathbb{I}_{W}\left(\mathcal{L}_{W}\{F\}\right)=\mathbb{I}_{W}\left(\mathcal{L}_{W}\{\mathrm{~N}\}\right)
$$

- for each $h \in \mathrm{N}$ a representation

$$
h=\sum_{i} a_{i} g_{\ell_{i}} \star \rho_{i}: a_{i} \in R \backslash\{0\}, \rho_{i} \in \mathcal{T}, g_{\ell_{i}} \in F,
$$

is called a standard representation in $\mathrm{R}$ in terms of $F$ iff

$$
v(h) \geq v\left(g_{\ell_{i}} \star \rho_{i}\right)=v\left(g_{\ell_{i}}\right) \circ \rho_{i}, \text { for each } i ;
$$

- if $u \in \operatorname{ker}\left(\mathfrak{s}_{W}\right)$ is $\mathcal{T}^{(m)}$-homogeneous and $U \in \operatorname{ker}\left(\Im_{W}\right)$ is such that $u=\mathcal{L}_{W}(U)$, we say that $u$ lifts to $U$, or $U$ is a lifting of $u$, or simply $u$ has a lifting;

- a restricted Gebauer-Möller set for $F$ is any $\mathcal{T}^{(m)}$-homogeneous basis of $\operatorname{ker}\left(\mathfrak{s}_{W}\right)$;

- for each $\mathcal{T}^{(m)}$-homogeneous element $\sigma \in\left(R \otimes_{\hat{R}} \mathrm{R}^{\text {op }}\right)^{u}$, we say that $\Im_{W}(\sigma)$ has a restricted quasi-Gröbner representation in terms of $G$ if it can be written as

$$
\widetilde{S}_{W}(\sigma)=\sum_{i} a_{i} g_{\ell_{i}} \star \rho_{i}: a_{i} \in R \backslash\{0\}, \rho_{i} \in \mathcal{T}, g_{\ell_{i}} \in F
$$

with $\mathbf{T}\left(g_{\ell_{i}}\right) \circ \rho_{i}<v(\sigma)$ for each $i$.

Theorem 40 (Möller-Pritchard). With the present notation and denoting $(5 \mathfrak{M}(F)$ any restricted Gebauer-Möller set for $F$, the following conditions are equivalent:

1. $F$ is a restricted Gröbner basis of $\mathbb{I}_{W}(F)$;

2. $f \in \mathbb{I}_{W}(F) \Longleftrightarrow f$ has a restricted Gröbner representation in terms of $F$;

3. for each $\sigma \in\left(\mathfrak{5 M}(F)\right.$, the restricted $S$-polynomial $\Im_{W}(\sigma)$ has a restricted quasiGröbner representation $\mathfrak{S}_{W}(\sigma)=\sum_{l=1}^{\mu} a_{l} g_{\ell_{l}} \star \rho_{l}$, in terms of $F$;

4. each $\sigma \in(\mathfrak{F M}(F)$ has a lifting $\operatorname{lift}(\sigma)$;

5. each $\mathcal{T}^{(m)}$-homogeneous element $u \in \operatorname{ker}\left(\mathfrak{s}_{W}\right)$ has a lifting $\operatorname{lift}(u)$.

Theorem 41 (Janet-Schreyer).

With the same notation the equivalent conditions (1-5) imply that

6. $\left\{\operatorname{lift}(\sigma): \sigma \in(\mathfrak{5 M}(F)\}\right.$ is a restricted standard basis of $\operatorname{ker}\left(\Im_{W}\right)$. 


\section{Gröbner basis Computation for Multivariate Ore Ex- tensions of Zacharias Domains}

We recall the definition of Zacharias ring [53], [30, §26.1], [31].

Definition 42. A ring $R$ with identity is called a (left) Zacharias ring if it satisfies the following properties:

(a). $\mathrm{R}$ is a noetherian ring;

(b). there is an algorithm which, for each $c \in R^{m}, C:=\left\{c_{1}, \ldots c_{t}\right\} \subset R^{m} \backslash\{0\}$, allows to decide whether $c \in \mathbb{I}_{L}(C)$ in which case it produces elements $d_{i} \in R: c=$ $\sum_{i=1}^{t} d_{i} c_{i}$

(c). there is an algorithm which, given $\left\{c_{1}, \ldots c_{t}\right\} \subset R^{m} \backslash\{0\}$, computes a finite set of generators for the left syzygy R-module $\left\{\left(d_{1}, \cdots, d_{t}\right) \in R^{t}: \sum_{i=1}^{t} d_{i} c_{i}=0\right\}$.

Note that [34] for a ring $R$ with identity which satisfies (a) and (b), (c) is equivalent to

(d). there is an algorithm which, given $\left\{c_{1}, \ldots c_{s}\right\} \subset R^{m} \backslash\{0\}$, computes a finite basis of the ideal

$$
\mathbb{I}_{L}\left(\left\{c_{i}: 1 \leq i<s\right\}\right): \mathbb{I}_{L}\left(c_{s}\right)
$$

If $R$ has canonical representatives, we improve the computational assumptions of Zacharias rings, requiring also the following property:

(e). there is an algorithm which, given an element $c \in R^{m}$ and a left module $\mathrm{J} \subset R^{m}$, computes the unique canonical representative $\operatorname{Rep}(c, \mathrm{~J})$.

If $R$ is a left Zacharias domain, the three algorithms proposed by Möller [34] for computing Gröbner bases in the polynomial ring over $R$ can be easily adapted to multivariate Ore extensions of Zacharias domains, provided that each $\alpha_{i}$, and therefore each $\alpha_{\tau}$, is an automorphism.

\subsection{First algorithm}

Still considering a finite basis

$$
F:=\left\{g_{1}, \ldots, g_{u}\right\} \subset \mathbf{R}^{m}, g_{i}=\mathbf{M}\left(g_{i}\right)-p_{i}=: c_{i} \tau_{i} \mathbf{e}_{l_{i}}-p_{i},
$$

of the module $\mathrm{M}:=\mathbb{I}_{L}(F)$ and denoting

$-\mathfrak{H}(F):=\left\{\left\{i_{1}, i_{2}, \ldots, i_{r}\right\} \subseteq\{1, \ldots, u\}: l_{i_{1}}=\cdots=l_{i_{r}}\right\} ;$

- for each $H:=\left\{i_{1}, i_{2}, \ldots, i_{r}\right\} \in \mathfrak{H}(F)$,

$$
\begin{aligned}
& -\varepsilon_{H}:=\mathbf{e}_{i_{1}}=\cdots=\mathbf{e}_{i_{1}}, \\
& -\tau_{H}:=\operatorname{lcm}\left(\tau_{i}: i \in H\right),
\end{aligned}
$$


- for each $I \subset H$,

$-\tau_{H, I}:=\frac{\tau_{H}}{\tau_{I}}$,

- $\alpha_{H, I}: R \rightarrow R$ the morphism $\alpha_{\tau_{H, l}}$;

- $\mathbf{T}(H):=\tau_{H} \varepsilon_{H}$,

and, if $R$ is a PID,

- $c_{H}:=\operatorname{lcm}\left(\alpha_{H, i}\left(c_{i}\right): i \in H\right)$,

- $\mu(H):=c_{H} \tau_{H}$ and

- $\mathbf{M}(H)=c_{H} \mathbf{T}(H)=c_{H} \tau_{H} \varepsilon_{H}=\mu(H) \varepsilon_{H} ;$

- $\mathbf{T}:=\{\mathbf{T}(H): H \in \mathfrak{H}(F)\}$

- for any $\mathrm{m}=\delta \epsilon \in \mathrm{T}$,

- for each $i, 1 \leq i \leq u, t_{i}(\mathrm{~m}):= \begin{cases}\frac{\delta}{\tau_{i}} & \text { if } \mathbf{T}\left(g_{i}\right) \mid \mathrm{m} \\ 1 & \text { otherwise; }\end{cases}$

- $v(\mathrm{~m})=\left(v(\mathrm{~m})_{1}, \ldots, v(\mathrm{~m})_{u}\right) \in R^{u}$ the vector such that

$$
v(\mathrm{~m})_{i}:= \begin{cases}\alpha_{t_{i}(\mathrm{~m})}\left(\operatorname{lc}\left(g_{i}\right)\right) & \text { if } \mathbf{T}\left(g_{i}\right) \mid \mathrm{m}, \\ 0 & \text { otherwise }\end{cases}
$$

$-C(\mathrm{~m}) \subset R^{u}$ a finite basis of the syzygy module

$$
\operatorname{Syz}_{L}\left(v(\mathrm{~m})_{1}, \ldots, v(\mathrm{~m})_{u}\right):=\left\{\left(d_{1}, \ldots, d_{u}\right) \in R^{u}: \sum_{i=1}^{u} d_{i} v(\mathrm{~m})_{i}=0\right\} ;
$$

- $S(\mathrm{~m}):=\left\{\left(d_{1} t_{1}(\mathrm{~m}), \ldots, d_{u} t_{u}(\mathrm{~m})\right):\left(d_{1}, \ldots, d_{u}\right) \in C(\mathrm{~m})\right\} ;$

- $\mathcal{S}(F):=\bigcup_{\mathrm{m} \in \mathrm{T}} S(\mathrm{~m})$;

- $\mathcal{S}^{\prime}(F) \subset \mathcal{S}(F)$ any subset satisfying

- for each $\sigma \in \mathcal{S}(F) \backslash \mathcal{S}^{\prime}(F)$ exist $\sigma_{j} \in \mathcal{S}^{\prime}(F), d_{j} \in R, \tau_{j} \in \mathcal{T}$, such that $\sigma=\sum_{j} d_{j} \tau_{j} * \sigma_{j}$

- $\mathcal{R}(F):=\left\{\sum_{i} m_{i} \star g_{i}:\left(m_{1}, \ldots, m_{u}\right) \in \mathcal{S}^{\prime}(F)\right\}$,

we have that (cf. [34], [30, Theorem 26.1.4])

Lemma 43. $\mathcal{S}(F)$ is a Gebauer-Möller set for $F$.

Proof. Let us consider a generic $\mathcal{T}^{(m)}$-homogeneus element

$$
\sigma:=\sum_{i=1}^{u} a_{i} \lambda_{i} e_{i} \in \mathrm{R}^{u} \backslash\{0\},
$$


with $a_{i} \in R, \lambda_{i} \in \mathcal{T}, v(\sigma):=\tau \epsilon$, and $a_{i} \neq 0 \Longrightarrow \lambda_{i} \tau_{i}=\tau, \epsilon=\mathbf{e}_{l_{i}}$, and assume that it is a left syzygy in $\operatorname{ker}\left(\mathfrak{s}_{L}\right)$.

Denoting $I:=\left\{i \leq u: a_{i} \neq 0\right\}$ and setting $\mathrm{m}:=\delta \epsilon:=\operatorname{lcm}\left\{\mathbf{T}\left(g_{i}\right): i \in H\right\} \mid v(\sigma)$, there is $v \in \mathcal{T}: v \delta=\tau$. With the present notation we also have $\delta=t_{i}(\mathrm{~m}) \tau_{i}$; thus $v t_{i}(\mathrm{~m}) \tau_{i}=v \delta=\lambda_{i} \tau_{i}$ and $\lambda_{i}=v t_{i}(\mathrm{~m})$. We also have

$$
0=\sum_{i=1}^{u} a_{i} \alpha_{\lambda_{i}}\left(\operatorname{lc}\left(g_{i}\right)\right)
$$

so that

$$
0=\alpha_{v}^{-1}\left(\sum_{i=1}^{u} a_{i} \alpha_{\lambda_{i}}\left(\operatorname{lc}\left(g_{i}\right)\right)\right)=\sum_{i=1}^{u} \alpha_{v}^{-1}\left(a_{i}\right) \alpha_{t_{i}(\mathrm{~m})}\left(\operatorname{lc}\left(g_{i}\right)\right),
$$

so that $\left(\alpha_{v}^{-1}\left(a_{1}\right), \ldots, \alpha_{v}^{-1}\left(a_{u}\right)\right) \in \operatorname{Syz}_{L}\left(v(\mathrm{~m})_{1}, \ldots, v(\mathrm{~m})_{u}\right)$.

Therefore, if we enumerate as

$$
\left(d_{11}, \ldots, d_{1 u}\right), \ldots,\left(d_{v 1}, \ldots, d_{v u}\right)
$$

a basis of $C(\mathrm{~m})$ and we denote $\mathrm{s}_{j}:=\sum_{i=1}^{u} d_{j i} t_{i}(\mathrm{~m}) e_{i}, 1 \leq j \leq v$, the elements of $S(\mathrm{~m})$, we have $\left(\alpha_{v}^{-1}\left(a_{1}\right), \ldots, \alpha_{v}^{-1}\left(a_{u}\right)\right)=\sum_{j=1}^{v} b_{j}\left(d_{j 1}, \ldots, d_{j u}\right)$ for suitable $b_{j} \in R$ and

$$
\begin{aligned}
\sigma & =\sum_{i=1}^{u} a_{i} \lambda_{i} e_{i} \\
& =\sum_{i=1}^{u} a_{i} v t_{i}(\mathrm{~m}) e_{i} \\
& =\sum_{i=1}^{u} v * \alpha_{v}^{-1}\left(a_{i}\right) t_{i}(\mathrm{~m}) e_{i} \\
& =v * \sum_{i=1}^{u} \sum_{j=1}^{v} b_{j} d_{j i} t_{i}(\mathrm{~m}) e_{i} \\
& =\sum_{j=1}^{v} \alpha_{v}\left(b_{j}\right) v *\left(\sum_{i=1}^{u} d_{j i} t_{i}(\mathrm{~m}) e_{i}\right) \\
& =\sum_{j=1}^{v} \alpha_{v}\left(b_{j}\right) v * \mathrm{~s}_{j} .
\end{aligned}
$$

Corollary 44. The following holds:

1. $\mathcal{S}^{\prime}(F)$ is a Gebauer-Möller set for $F$.

2. $F$ is a left Gröbner basis of the module it generates iff each $h \in \mathcal{R}(F)$ has a left Gröbner representation in terms of $F$. 
Example 45. If we consider the ring of Example 17 as a left $\mathbb{Z}[x]$-module endowed with the $\Gamma$-pseudovaluation, $\Gamma=\left\{Y_{1}^{a_{1}} Y_{2}^{a_{2}} Y_{3}^{a_{3}}:\left(a_{1}, a_{2}, a_{1}\right) \in \mathbb{N}^{3}\right\}$, we obtain a similar solution as the one described in Example 35.

Expressing each $\mathbf{M}\left(f_{i}\right)$ as $\mathbf{M}\left(f_{i}\right)=\operatorname{lc}\left(f_{i}\right) \mathbf{T}\left(f_{i}\right)$, according Zacharias approach we need to compute a syzygy bases in $\mathbb{Z}[x]$ among $\alpha_{Y_{1}}\left(\operatorname{lc}\left(f_{1}\right)\right)=\left(y^{2}-1\right), \alpha_{Y_{2}}\left(\operatorname{lc}\left(f_{2}\right)\right)=$ $\left(y^{3}-1\right)$ and $\alpha_{Y_{3}}\left(\operatorname{lc}\left(f_{3}\right)\right)=\left(y^{4}-1\right)$; the natural solutions $\left(-\left(y^{2}+y+1\right),(y+1), 0\right)$, $\left(-\left(y^{2}-1\right), 0,1\right)$ produce $\sigma_{1}$ and $\sigma_{2}$.

Example 46. Let us now specialize the ring of Example 14to the case

$$
n=3, e_{1}=e_{2}=e_{3}=1, c_{1}=20, c_{2}=6, c_{3}=15,
$$

and let us consider four elements $f_{1}, f_{2}, f_{3}, f_{4} \in \mathrm{R}$ with

$$
\mathbf{M}\left(f_{1}\right)=x Y_{1} Y_{2}^{3} Y_{3}^{2}, \mathbf{M}\left(f_{2}\right)=x^{2} Y_{1}^{2} Y_{2} Y_{3}^{2}, \mathbf{M}\left(f_{3}\right)=x Y_{1}^{2} Y_{2}^{3} Y_{3}, \mathbf{M}\left(f_{4}\right)=x Y_{1}^{2} Y_{2}^{2} Y_{3}^{2} .
$$

We have

$$
\mathrm{T}=\left\{Y_{1} Y_{2}^{3} Y_{3}^{2}, Y_{1}^{2} Y_{2} Y_{3}^{2}, Y_{1}^{2} Y_{2}^{2} Y_{3}^{2}, Y_{1}^{2} Y_{2}^{3} Y_{3}, Y_{1}^{2} Y_{2}^{3} Y_{3}^{2}\right\}
$$

and

\begin{tabular}{lll}
$\mathrm{m}$ & $\left(t_{1}(\mathrm{~m}), \ldots, t_{4}(\mathrm{~m})\right)$ & $v(\mathrm{~m})$ \\
\hline$Y_{1} Y_{2}^{3} Y_{3}^{2}$ & $(1,0,0,0)$ & $(x, 0,0,0)$ \\
$Y_{1}^{2} Y_{2} Y_{3}^{2}$ & $(0,1,0,0)$ & $\left(0, x^{2}, 0,0\right)$ \\
$Y_{1}^{2} Y_{2}^{2} Y_{3}^{2}$ & $\left(0, Y_{2}, 0,1\right)$ & $\left(0,6^{2} x^{2}, 0, x\right)$ \\
$Y_{1}^{2} Y_{2}^{3} Y_{3}$ & $(0,0,1,0)$ & $(0,0, x, 0)$ \\
$Y_{1}^{2} Y_{2}^{3} Y_{3}^{2}$ & $\left(Y_{1}, Y_{2}^{2}, Y_{3}, Y_{2}\right)$ & $\left(20 x, 6^{4} x^{2}, 15 x, 6 x\right)$,
\end{tabular}

Denoting

$$
\begin{aligned}
& b(1,3):=\left(-3 Y_{1}, 0,4 Y_{3}, 0\right), \\
& b(2,4):=\left(0,-Y_{2}, 0,6^{2} x\right), \\
& b(3,4):=\left(0,0,-2 Y_{3}, 0,5 Y_{2}\right)
\end{aligned}
$$

we have $S\left(Y_{1}^{2} Y_{2}^{2} Y_{3}^{2}\right)=\{b(2,4)\}$ and, since

$$
Y_{2} * b(2,4)=\left(0,-Y_{2}^{2}, 0,6^{2} Y_{2} * x\right)=\left(0,-Y_{2}^{2}, 0,6^{3} x Y_{2}\right)
$$

we can take $S\left(Y_{1}^{2} Y_{3}^{2} Y_{3}^{2}\right)=\left\{b(1,3), Y_{2} * b(2,4), b(3,4)\right\}$; thus

$$
\mathcal{S}^{\prime}:=\{b(1,3), b(2,4), b(3,4)\}
$$

is the required Gebauer-Möller set.

\subsection{Second algorithm}

Möller proposes an (essentially) equivalent alternative computation: for any $s, 1 \leq s \leq$ $u$, let us consider the syzygy module

$$
\mathcal{S}_{s}:=\left\{\left(h_{1}, \ldots, h_{s}\right): \sum_{i=1}^{s} h_{i} \star \mathbf{M}\left(g_{i}\right)=0\right\} \subset \mathbf{R}^{s}
$$


and let us compute $\mathcal{S}(F)=\mathcal{S}_{u}$ by inductively extending $\mathcal{S}_{s-1}$ to $\mathcal{S}_{s}$, the inductive seed being $\mathcal{S}_{1}=\emptyset$.

A direct application of the property (d) of a Zacharias ring allows to compute a Gebauer-Möller set via

Definition 47. A subset $H \subset\{1, \ldots, s\} \cap \mathfrak{H}(F), s \leq u$, is said to be maximal for a term $\delta \epsilon \in \mathcal{T}^{(m)}$ if $H=\left\{i, 1 \leq i \leq s: \tau_{i} \mid \delta, \mathbf{e}_{l_{i}}=\epsilon\right\}$, basic if $s \in H$ and $H$ is maximal for $\mathbf{T}(H)$.

For a basic subset $H \subset\{1, \ldots, s\} \cap \mathfrak{S}(F)$, denote $H^{\times}:=H \backslash\{s\}$. For any

$$
d_{s} \in \mathbb{I}_{L}\left(\left\{\alpha_{H, i}\left(c_{i}\right): i \in H^{\times}\right\}\right): \mathbb{I}_{L}\left(\alpha_{H, s}\left(c_{s}\right)\right),
$$

a syzygy associated to $H$ and $d_{s}$ is any $\mathcal{T}^{(m)}$-homogeneous syzygy

$$
\sum_{i \in H^{\times}} d_{i} \frac{\tau_{H}}{\tau_{i}} e_{i}+d_{s} \frac{\tau_{H}}{\tau_{s}} e_{s} \in \mathcal{S}_{s}
$$

where $d_{i} \in R$ are suitable elements for which $d_{s} \alpha_{H, s}\left(c_{s}\right)=-\sum_{i \in H^{\times}} d_{i} \alpha_{H, i}\left(c_{i}\right)$.

Theorem 48 (Möller). [34] With the present notation, denoting

- $\left\{A_{1}, \ldots, A_{\mu}\right\}$ a $\mathcal{T}^{(m)}$-homogeneous basis of $\mathcal{S}_{s-1}$,

- $\mathcal{H}$ the set of all basic subsets $H \subset\{1, \ldots, s\} \cap \mathfrak{H}(F)$,

- $\left\{d_{1 H}, \ldots, d_{r_{H} H}\right\}$ a basis of the ideal $\mathbb{I}_{L}\left(\left\{\alpha_{H, i}\left(c_{i}\right)\right.\right.$ s.t. $\left.\left.i \in H^{\times}\right\}\right): \mathbb{I}_{L}\left(\alpha_{H, s}\left(c_{s}\right)\right)$ for each basic subset $H \in \mathcal{H}$,

- $D_{j H} \in \mathrm{R}^{s}$ a syzygy associated to $H$ and $d_{j H}$, for each basic subset $H \in \mathcal{H}$ and each $j, 1 \leq j \leq r_{H}$

the set $\left\{A_{1}, \ldots, A_{\mu}\right\} \cup\left\{D_{j H}: H \in \mathcal{H}, 1 \leq j \leq r_{H}\right\}$ is a $\mathcal{T}^{(m)}$-homogeneous basis of $\mathcal{S}_{s}$.

Proof. Let $S:=\left(d_{1} \lambda_{1}, \ldots, d_{s} \lambda_{s}\right) \in \mathcal{S}_{s}, d_{s} \neq 0$, be a $\mathcal{T}^{(m)}$-homogeneous element of $\mathcal{T}^{(m)}$-degree $\delta \epsilon$ and let

$$
K:=\left\{i, 1 \leq i \leq s: d_{i} \neq 0\right\}
$$

since by $\mathcal{T}^{(m)}$-homogeneity, $\tau_{i} \mid \delta$ and $\mathbf{e}_{t_{i}}=\epsilon$ for each $i \in K$, we have $\mathbf{T}(K) \mid \delta \epsilon$; we also have $d_{i}=0$ for each $i \notin K$ and $\lambda_{i} \tau_{i}=\delta, \mathbf{e}_{t_{i}}=\epsilon$ for each $i \in K$.

For the set $H:=\left\{i, 1 \leq i \leq s: \tau_{i} \mid \tau_{K}, \mathbf{e}_{l_{i}}=\varepsilon_{K}\right\}$ clearly we have $\tau_{H} \mid \tau_{K}$ and $K \subseteq H$ so that $\tau_{H}\left|\tau_{K}\right| \delta$; we also have $\varepsilon_{H}=\varepsilon_{K}=\epsilon$. Moreover $d_{s} \neq 0$ implies $s \in K \subseteq H$ so that $H$ is basic. Since $\left(d_{1} \lambda_{1}, \ldots, d_{s} \lambda_{s}\right) \in \mathcal{S}_{s}$, setting $v:=\frac{\delta}{\tau_{H}}$, we have

$$
0=\sum_{i=1}^{s} d_{i} \lambda_{i} * \mathbf{M}\left(g_{i}\right)=\sum_{i \in H} d_{i} \frac{\delta}{\tau_{i}} * c_{i} \tau_{i} \epsilon=\left(\sum_{i \in H} d_{i} \alpha_{\lambda_{i}}\left(c_{i}\right)\right) \delta \epsilon
$$

so that $\sum_{i \in H} d_{i} \alpha_{\lambda_{i}}\left(c_{i}\right)=0, \sum_{i \in H} \alpha_{v}^{-1}\left(d_{i}\right) \alpha_{H, i}\left(c_{i}\right)=0$, whence $\alpha_{v}^{-1}\left(d_{s}\right) \alpha_{H, s}\left(c_{s}\right) \in \mathbb{I}_{L}\left(\alpha_{H, i}\left(c_{i}\right): i \in H^{\times}\right)$and $\alpha_{v}^{-1}\left(d_{s}\right) \in \mathbb{I}_{L}\left(\alpha_{H, i}\left(c_{i}\right): i \in H^{\times}\right): \mathbb{I}_{L}\left(\alpha_{H, s}\left(c_{s}\right)\right)$.

Therefore $\alpha_{v}^{-1}\left(d_{s}\right)=\sum_{j=1}^{r_{H}} u_{j} d_{j H}$ and $S-\sum_{j=1}^{r_{H}} \alpha_{v}\left(u_{j}\right) v * D_{j H} \in \mathcal{S}_{s-1}$. 
Example 49. If we consider the ring of Example 17 as a left $\mathbb{Z}[x]$-module endowed with the $\Gamma$-pseudovaluation, $\Gamma=\left\{Y_{1}^{a_{1}} Y_{2}^{a_{2}} Y_{3}^{a_{3}}:\left(a_{1}, a_{2}, a_{1}\right) \in \mathbb{N}^{3}\right\}$, we obtain a similar solution as the one described in Example 35.

Expressing each $\mathbf{M}\left(f_{i}\right)$ as $\mathbf{M}\left(f_{i}\right)=\operatorname{lc}\left(f_{i}\right) \mathbf{T}\left(f_{i}\right)$, according Zacharias approach we need to compute a syzygy bases in $\mathbb{Z}[x]$ among $\alpha_{Y_{1}}\left(\operatorname{lc}\left(f_{1}\right)\right)=\left(y^{2}-1\right), \alpha_{Y_{2}}\left(\operatorname{lc}\left(f_{2}\right)\right)=$ $\left(y^{3}-1\right)$ and $\alpha_{Y_{3}}\left(\operatorname{lc}\left(f_{3}\right)\right)=\left(y^{4}-1\right)$; the natural solutions $\left(-\left(y^{2}+y+1\right),\left(y^{2}+1\right), 0\right)$, $\left(-\left(y^{2}-1\right), 0,1\right)$ produce $\sigma_{1}$ and $\sigma_{3}$.

Example 50. In Examples 46, the basic elements are the following:

\begin{tabular}{l|ll|l} 
& $H$ & $\mathbf{T}(H)$ & $f_{H}$ \\
\hline$s=1$ & $\{1\}$ & $Y_{1} Y_{2}^{3} Y_{3}^{2}$ & $f_{1}$ \\
\hline$s=2$ & $\{1\}$ & $Y_{1} Y_{2}^{3} Y_{3}^{2}$ & $f_{1}$ \\
& $\{2\}$ & $Y_{1}^{2} Y_{2} Y_{3}^{2}$ & $f_{2}$ \\
& $\{1,2\}$ & $Y_{1}^{2} Y_{2}^{3} Y_{3}^{2}$ & $f_{\{1,2\}}=4 x Y_{1}^{2} Y_{2}^{3} Y_{3}^{2}$, \\
\hline$s=3$ & $\{1\}$ & $Y_{1} Y_{2}^{3} Y_{3}^{2}$ & $f_{1}$ \\
& $\{2\}$ & $Y_{1}^{2} Y_{2} Y_{3}^{2}$ & $f_{2}$ \\
& $\{3\}$ & $Y_{1}^{2} Y_{2}^{3} Y_{3}$ & $f_{3}$ \\
& $\{1,2,3\}$ & $Y_{1}^{2} Y_{2}^{3} Y_{3}^{2}$ & $f_{\{1,2,3\}}=x Y_{1}^{2} Y_{2}^{3} Y_{3}^{2}$, \\
\hline$s=4$ & $\{1\}$ & $Y_{1} Y_{2}^{3} Y_{3}^{2}$ & $f_{1}$ \\
& $\{2\}$ & $Y_{1}^{2} Y_{2} Y_{3}^{2}$ & $f_{2}$ \\
& $\{3\}$ & $Y_{1}^{2} Y_{2}^{3} Y_{3}$ & $f_{3}$ \\
& $\{2,4\}$ & $Y_{1}^{2} Y_{2}^{2} Y_{3}^{2}$ & $f_{\{2,4\}}=f_{4}$ \\
& $\{1,2,3,4\}$ & $Y_{1}^{2} Y_{2}^{3} Y_{3}^{2}$ & $f_{\{1,2,3,4\}}=f_{\{1,2,3\}}$.
\end{tabular}

Corollary 51. Assuming that the Zacharias domain $R$ is a principal ideal domain and denoting, for each $i, j, 1 \leq i<j \leq u, \mathbf{e}_{t_{i}}=\mathbf{e}_{t_{j}}$,

$$
\begin{aligned}
b(i, j) & :=\frac{\operatorname{lcm}\left(\alpha_{\{i, j\}, i}\left(c_{i}\right), \alpha_{\{i, j\}, j}\left(c_{j}\right)\right)}{\alpha_{\{i, j\}, j}\left(c_{j}\right)} \frac{\operatorname{lcm}\left(\tau_{i}, \tau_{j}\right)}{\tau_{j}} e_{j} \\
& -\frac{\operatorname{lcm}\left(\alpha_{\{i, j\}, i}\left(c_{i}\right), \alpha_{\{i, j\}, j}\left(c_{j}\right)\right)}{\alpha_{\{i, j\}, i}\left(c_{i}\right)} \frac{\operatorname{lcm}\left(\tau_{i}, \tau_{j}\right)}{\tau_{i}} e_{i}, \\
B(i, j) & :=\frac{\operatorname{lcm}\left(\alpha_{\{i, j\}, i}\left(c_{i}\right), \alpha_{\{i, j\}, j}\left(c_{j}\right)\right)}{\alpha_{\{i, j\}, j}\left(c_{j}\right)} \frac{\operatorname{lcm}\left(\tau_{i}, \tau_{j}\right)}{\tau_{j}} \star g_{j} \\
& -\frac{\operatorname{lcm}\left(\alpha_{\{i, j\}, i}\left(c_{i}\right), \alpha_{\{i, j\}, j}\left(c_{j}\right)\right)}{\alpha_{\{i, j\}, i}\left(c_{i}\right)} \frac{\operatorname{lcm}\left(\tau_{i}, \tau_{j}\right)}{\tau_{i}} \star g_{i}
\end{aligned}
$$

we have that $\left\{b(i, j): 1 \leq i<j \leq u, \mathbf{e}_{t_{i}}=\mathbf{e}_{\iota_{j}}\right\}$ is a Gebauer-Möller set for $F$, so that $F$ is a Gröbner basis of $\mathrm{M}$, iff each $B(i, j), 1 \leq i<j \leq u, \mathbf{e}_{i}=\mathbf{e}_{\iota_{j}}$, has a weak Gröbner representation in terms of $F$.

\footnotetext{
${ }^{2}$ Remember that $\alpha_{\{i, j\}, j}=\alpha_{\tau}$ for $\tau=\frac{\operatorname{lcm}\left(\tau_{i}, \tau_{j}\right)}{\tau_{j}}$.
} 
Proof. Since, for any basic subset $H \subset\{1, \ldots, s\} \cap \mathfrak{S}(F)$ we have

$$
\begin{aligned}
\mathbb{I}\left(\left\{\alpha_{\{i, s\}, i}\left(c_{i}\right): i \in H^{\times}\right\}\right): \mathbb{I}\left(\alpha_{\{i, s\}, s}\left(c_{s}\right)\right) & =\bigoplus\left(\mathbb{I}\left(\alpha_{\{i, s\}, i}\left(c_{i}\right)\right): \mathbb{I}\left(\alpha_{\{i, s\}, s}\left(c_{s}\right)\right)\right) \\
& =\mathbb{I}\left(\frac{\operatorname{lcm}\left(\alpha_{\{i, s\}, i}\left(c_{i}\right), \alpha_{\{i, s\}, s}\left(c_{s}\right)\right)}{\alpha_{\{i, s\}, s}\left(c_{s}\right)}\right)
\end{aligned}
$$

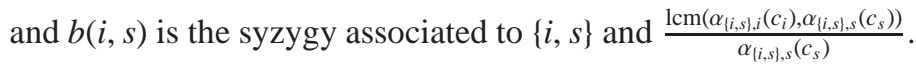

Example 52. In Examples 46, we obtain the following redundant Gebauer-Möller set (see Examples 65)

\begin{tabular}{lllrrrr}
$(i, j)$ & $\operatorname{lcm}\left(\alpha_{\{i, j\}, i}\left(c_{i}\right), \alpha_{\{i, j\}, j}\left(c_{j}\right)\right)$ & $\operatorname{lcm}\left(\tau_{i}, \tau_{j}\right)$ & $b(i, j)$ & & & \\
\hline$(1,2)$ & $6^{4} \cdot 5 x^{2}$ & $Y_{1}^{2} Y_{2}^{3} Y_{3}^{2}$ & $\left(-2^{2} 3^{4} x Y_{1}\right.$, & $5 Y_{2}^{2}$, & 0, & $0)$ \\
$(1,3)$ & $60 x$ & $Y_{1}^{2} Y_{2}^{3} Y_{3}^{2}$ & $\left(-3 Y_{1}\right.$, & 0, & $4 Y_{3}$, & $0)$ \\
$(2,3)$ & $6^{4} \cdot 5 x^{2}$ & $Y_{1}^{2} Y_{2}^{3} Y_{3}^{2}$ & $(0$, & $-5 Y_{2}^{2}$, & $3^{3} 2^{4} x Y_{3}$, & $0)$ \\
$(1,4)$ & $60 x$ & $Y_{1}^{2} Y_{2}^{3} Y_{3}^{2}$ & $\left(-3 Y_{1}\right.$, & 0, & 0, & $\left.10 Y_{2}\right)$ \\
$(2,4)$ & $6^{2} x^{2}$ & $Y_{1}^{2} Y_{2}^{2} Y_{3}^{2}$ & $(0$, & $-Y_{2}$, & 0, & $\left.6^{2} x\right)$ \\
$(3,4)$ & $30 x$ & $Y_{1}^{2} Y_{2}^{3} Y_{3}^{2}$ & $(0$, & 0, & $-2 Y_{3}$, & $\left.5 Y_{2}\right)$
\end{tabular}

Corollary 53. Assuming that the Zacharias domain $R$ is a principal ideal domain and that each $\alpha_{i}$ is an automorphism denoting, for each $i, j, 1 \leq i<j \leq u, \mathbf{e}_{t_{i}}=\mathbf{e}_{t_{j}}$,

$$
\begin{aligned}
b(i, j) & :=e_{j} \alpha_{\tau_{j}}^{-1}\left(\frac{\operatorname{lcm}\left(c_{i}, c_{j}\right)}{c_{j}}\right) \frac{\operatorname{lcm}\left(\tau_{i}, \tau_{j}\right)}{\tau_{j}}-e_{i} \alpha_{\tau_{i}}^{-1}\left(\frac{\operatorname{lcm}\left(c_{i}, c_{j}\right)}{c_{i}}\right) \frac{\operatorname{lcm}\left(\tau_{i}, \tau_{j}\right)}{\tau_{i}} \\
B(i, j) & :=g_{j} \star \alpha_{\tau_{j}}^{-1}\left(\frac{\operatorname{lcm}\left(c_{i}, c_{j}\right)}{c_{j}}\right) \frac{\operatorname{lcm}\left(\tau_{i}, \tau_{j}\right)}{\tau_{j}}-g_{i} \star \alpha_{\tau_{i}}^{-1}\left(\frac{\operatorname{lcm}\left(c_{i}, c_{j}\right)}{c_{i}}\right) \frac{\operatorname{lcm}\left(\tau_{i}, \tau_{j}\right)}{\tau_{i}}
\end{aligned}
$$

we have that $\left\{b(i, j): 1 \leq i<j \leq u, \mathbf{e}_{t_{i}}=\mathbf{e}_{\iota_{j}}\right\}$ is a Gebauer-Möller set for $F$, so that $F$ is a right Gröbner basis of $\mathrm{M}$, iff each $B(i, j), 1 \leq i<j \leq u, \mathbf{e}_{l_{i}}=\mathbf{e}_{l_{j}}$, has a right weak Gröbner representation in terms of $F$.

\subsection{Third algorithm: from weak to strong Gröbner basis}

As regards strong Gröbner bases, we have

Definition 54. A set $C \subset \mathrm{R}^{m}$ is called a completion of $F$, if, for each subset $H \subset \mathfrak{H}(F)$ which is maximal for $\mathbf{T}(H)$, it contains an element $f_{H} \in \mathbb{I}(F)$ which satisfies

1. $\mathbf{T}\left(f_{H}\right)=\mathbf{T}(H)=\tau_{H} \varepsilon_{H}$,

2. $\operatorname{lc}\left(f_{H}\right)=c_{H}=\operatorname{gcd}\left(\alpha_{H, i}\left(\operatorname{lc}\left(g_{i}\right)\right): i \in H\right)$,

3. $f_{H}$ has a Gröbner representation in terms of $F$. 
Algorithm 55 (Möller). A completion of $F$ can be inductively computed by mimicking the construction of Theorem 48 as follows: the result being trivial if $\# F=1$, we can assume to have already obtained a completion $C\left(F^{\times}\right)$of $F^{\times}=\left\{g_{1}, \ldots, g_{s-1}\right\}, s \leq u$; for each maximal subset $H \subset\{1, \ldots, s\}$, if $s \notin H$ we can take as $f_{H}$ the corresponding element in $C\left(F^{\times}\right)$. If instead $s \in H$, then $H^{\times}$is maximal in $F^{\times}$for $\mathbf{T}\left(H^{\times}\right)$and $\tau_{H^{\times}} \mid \tau_{H}$; thus there is a corresponding element $f_{H^{\times}}$in $C\left(F^{\times}\right)$; let us compute the values $s, t, d \in R$ such that

$$
\alpha_{H, H^{\times}}\left(\operatorname{lc}\left(f_{H^{\times}}\right)\right) s+\alpha_{H, s}\left(\operatorname{lc}\left(g_{s}\right)\right) t=\operatorname{gcd}\left(\alpha_{H, H^{\times}}\left(\operatorname{lc}\left(f_{H^{\times}}\right)\right), \alpha_{H, s}\left(\operatorname{lc}\left(g_{s}\right)\right)\right)=d
$$

and define $f_{H}:=s \frac{\tau_{H}}{\tau_{H^{\times}}} \star f_{H^{\times}}+t \frac{\tau_{H}}{\tau_{s}} \star g_{s}$ which satisfies $\mathbf{M}\left(f_{H}\right)=d \mathbf{T}(H)=d \tau_{H} \epsilon_{H}$ so that

1. $\mathbf{T}\left(f_{H}\right)=\mathbf{T}(H)=\tau_{H} \epsilon_{H}$,

2. $\operatorname{lc}\left(f_{H}\right)=\operatorname{gcd}\left(\alpha_{H, H^{\times}}\left(\operatorname{lc}\left(f_{H^{\times}}\right)\right), \alpha_{H, s}\left(\operatorname{lc}\left(g_{s}\right)\right)\right)=\operatorname{gcd}\left(\alpha_{H, i}\left(\operatorname{lc}\left(g_{i}\right)\right): i \in H\right)=d$;.

3. it is sufficient to substitute $f_{H^{\times}}$with its Gröbner representation, to obtain the required Gröbner representation of $f_{H}$.

Proposition 56 (Möller). With the present notation and under the assumption that $R$ is a principal ideal domain, the following conditions are equivalent:

\section{F is a Gröbner basis of $\mathrm{M}$;}

2. a completion of $F$ is a strong Gröbner basis of $\mathrm{M}$.

Proof.

(1) $\Longrightarrow$ (2) Let $f \in \mathrm{M}$ and let $f=\sum_{i=1}^{u} h_{i} \star g_{i}$ be a Gröbner representation; denoting $H:=\left\{j: \mathbf{T}\left(h_{j} \star g_{j}\right)=\mathbf{T}(f)=: \tau \epsilon\right\}$ we have $\tau_{H} \mid \tau, \epsilon_{H}=\epsilon$. Thus, setting $v_{j}:=\frac{\tau}{\tau_{j}}, \omega_{j}:=\frac{\tau_{H}}{\tau_{j}}$ for each $j$ and $\lambda:=\frac{\tau}{\tau_{H}}$ we have

$$
\begin{aligned}
\operatorname{lc}(f)=\sum_{j \in H} \operatorname{lc}\left(h_{j}\right) \alpha_{v_{j}}\left(\operatorname{lc}\left(g_{j}\right)\right) & \\
=\sum_{j \in H} \operatorname{lc}\left(h_{j}\right) \alpha_{\lambda} \alpha_{\omega_{j}}\left(\operatorname{lc}\left(g_{j}\right)\right) & \in \mathbb{I}\left(\alpha_{\lambda} \alpha_{\omega_{j}}\left(\operatorname{lc}\left(g_{j}\right)\right): j \in H\right) \\
& =\alpha_{\lambda}\left(\mathbb{I}\left(\alpha_{\omega_{j}}\left(\operatorname{lc}\left(g_{j}\right)\right): j \in H\right)\right) \\
& =\alpha_{\lambda}\left(\mathbb{I}\left(c_{H}\right)\right)
\end{aligned}
$$

so that $\alpha_{\lambda}\left(\operatorname{lc}\left(f_{H}\right)\right)=\alpha_{\lambda}\left(c_{H}\right) \mid \operatorname{lc}(f)$ and $\operatorname{lc}(f)=d \alpha_{\lambda}\left(\operatorname{lc}\left(f_{H}\right)\right)$ with $d \in R$. In conclusion we have $\mathbf{M}(f)=d \lambda * \mathbf{M}\left(f_{H}\right)$.

(2) $\Longrightarrow$ (1) Let $f \in \mathrm{M}$ and let $f=\sum_{K \subset \mathfrak{\mathfrak { S }}(F)} c_{K} \tau_{K} f_{K}$ be a strong Gröbner representation of it in terms of a completion of $F$; it is sufficient to substitute each $f_{K}$ with a Gröbner representation of it in terms of $F$ to obtain the required representation.

Example 57. In the ring of Examples 17 and 35, we finally have (see Remark 36.

$$
f_{\{1,2\}}=f_{\{1,3\}}=f_{\{1,2,3\}}=\mathfrak{s}_{L}\left(\varsigma_{A}\right)=\mathfrak{s}_{L}\left(\varsigma_{B}\right)=(y-1) Y_{1}^{2} Y_{2}^{2} Y_{3}^{2} .
$$


Example 58. The strong Grø"bner basis (see Examples 50) is

$$
\left\{f_{1}, f_{2}, f_{3}, f_{4}, f_{\{1,2,3,4\}}\right\}
$$

since

$$
\begin{gathered}
\left.\left.\left.\operatorname{gcd}\left(\alpha_{\{1,2\},\{1\}}\left(\operatorname{lc}\left(f_{1}\right)\right), \alpha_{\{1,2\}, 2}\left(\operatorname{lc}\left(f_{2}\right)\right)\right)=\operatorname{gcd}\left(\alpha_{Y_{1}}(x)\right), \alpha_{Y_{2}^{2}}(x)\right)=\operatorname{gcd}(20 x)\right), 36 x\right)=4 x \\
\left.\left.\left.\operatorname{gcd}\left(\alpha_{\{1,2,3\},\{1,2\}}\left(\operatorname{lc}\left(f_{\{1,2\}}\right)\right), \alpha_{\{1,2,3\}, 3}\left(\operatorname{lc}\left(f_{3}\right)\right)\right)=\operatorname{gcd}\left(\alpha_{1}(4 x)\right), \alpha_{Y_{3}}(x)\right)=\operatorname{gcd}(4 x)\right), 15 x\right)=x .
\end{gathered}
$$

Similarly, $f_{\{2,4\}}=f_{4}$ follows trivially from

$$
\left.\left.\left.\operatorname{gcd}\left(\alpha_{\{2,4\},\{2\}}\left(\operatorname{lc}\left(f_{2}\right)\right), \alpha_{\{2,4\}, 4}\left(\operatorname{lc}\left(f_{4}\right)\right)\right)=\operatorname{gcd}\left(\alpha_{Y_{2}}\left(x^{2}\right)\right), \alpha_{1}(x)\right)=\operatorname{gcd}\left(36 x^{2}\right)\right), x\right)=x .
$$

\subsection{Useless S-pairs and Gebauer-Möller sets}

Let us still assume that the Zacharias domain $R$ is a principal ideal domain and we will use freely notations as $\mathbf{M}(i), \mathbf{M}(i, j), \mathbf{M}(i, j, k), 1 \leq i, j, k \leq u$, instead of $\mathbf{M}(\{i\})$, $\mathbf{M}(\{i, j\}), \mathbf{M}(\{i, j, k\})$; we can then easily apply to the present setting the reformulation and improvement by Gebauer-Möller [18] of Buchberger Criteria [9]. However we must be aware that in this context, there is no chance of reformulating Buchberger's First Criterion.

Remark 59. In fact we should at least require that

$$
\mathbf{M}(i) * \mathbf{M}(j)=\mathbf{M}(i) * \mathbf{M}(j)=\mathbf{M}(i, j)
$$

id est not only $\operatorname{lcm}\left(\tau_{i}, \tau_{j}\right)=\tau_{i} \circ \tau_{j}=\tau_{j} \circ \tau_{i}$ which is trivially true but also

$$
\operatorname{lcm}\left(\alpha_{\tau_{j}}\left(c_{i}\right), \alpha_{\tau_{i}}\left(c_{j}\right)\right)=c_{j} \alpha_{\tau_{j}}\left(c_{i}\right)=c_{i} \alpha_{\tau_{i}}\left(c_{j}\right) .
$$

This essentially requires $c_{i} \mid \alpha_{\tau_{j}}\left(c_{i}\right)$ and $c_{j} \mid \alpha_{\tau_{i}}\left(c_{j}\right)$ whence $\alpha_{\tau_{j}}=$ Id; this suggests that Buchberger's First Criterion hardly can be applied except for the case of the commutative ring $\mathcal{P}=R\left[Y_{1}, \ldots, Y_{n}\right], R$ a PIR, where it is stated as

If $F \subset \mathcal{P}$ and $\mathbb{I}(F)$ is an ideal of $\mathcal{P}$, it holds

$$
\begin{aligned}
& \mathbf{M}(i) \mathbf{M}(j)=\mathbf{M}(i, j) \Longleftrightarrow \quad \operatorname{cm}\left(\tau_{i}, \tau_{j}\right)=\tau_{i} \tau_{j}, \operatorname{lcm}\left(c_{i}, c_{j}\right)=c_{i} c_{j} \\
& \Longrightarrow \\
& \operatorname{NF}(B(i, j), F)=0 .
\end{aligned}
$$

Note that the proof which considers the trival sysygies $g_{i} g_{j}-g_{j} g_{i}=0$ holds only to the classical polynomial ring case.

Definition 60. A useful S-pair set for $F$ is any subset

$$
\mathfrak{5} \mathfrak{M} \subset \mathcal{S}(u)=\left\{\{i, j\}, 1 \leq i<j \leq u, \mathbf{e}_{l_{i}}=\mathbf{e}_{l_{j}}\right\}
$$

such that $\{b(i, j):\{i, j\} \in(\mathfrak{5} \mathfrak{M}\}$ is a Gebauer-Möller set for $F$. 
Corollary 61. With the present notation, under the assumption that $R$ is a principal ideal domain, $F$ is a Gröbner basis of the left module $\mathrm{M}$ iff, denoting (5MM a useful $S$-pair set for $F$, each S-polynomial B(i,j), $\{i, j\} \in \mathbb{5 M}$ has a Gröbner representation in terms of $F$.

Proof. By definition $\{b(i, j):\{i, j\} \in(\mathfrak{5 M M}\}$ is a Gebauer-Möller set for $F$ so that, by Theorem 33, $F$ is a Gröbner basis of M iff each S-polynomial $B(i, j),\{i, j\} \in \mathfrak{5} \mathfrak{M}$ has a Gröbner representation in terms of $F$.

If we moreover define,

- for each $i, j: 1 \leq i, j \leq u, \mathbf{e}_{l_{i}}=\mathbf{e}_{l_{j}}$,

$$
\text { - } c(i, j):=\operatorname{lcm}\left(\alpha_{\{i, j\}, i}\left(c_{i}\right), \alpha_{\{i, j\}, j}\left(c_{j}\right)\right),
$$

- $\tau(i, j)=\operatorname{lcm}\left(\tau_{i}, \tau_{j}\right)$

$-\mu(i, j)=c(i, j) \tau(i, j)$

- and for each $i, j, k: 1 \leq i, j, k \leq u, \mathbf{e}_{l_{i}}=\mathbf{e}_{l_{j}}=\mathbf{e}_{l_{k}}$,

- $c(i, j, k):=\operatorname{lcm}\left(\alpha_{\{i, j, k\},\{i, j\}}(c(i, j)), \alpha_{\{i, j, k\},\{i, k\}}(c(i, k)), \alpha_{\{i, j, k\} ; j, k\}}(c(j, k))\right)$

- $\tau(i, j, k)=\operatorname{lcm}\left(\tau_{i}, \tau_{j}, \tau_{k}\right)$

- $\mu(i, j, k)=c(i, j, k) \tau(i, j, k)$,

and we impose on the set

$$
\mathcal{S}(u):=\left\{\{i, j\}, 1 \leq i<j \leq u, \mathbf{e}_{l_{i}}=\mathbf{e}_{l_{j}}\right\}
$$

the ordering $<$ defined by

$$
\left\{i_{1}, j_{1}\right\}<\left\{i_{2}, j_{2}\right\} \Longleftrightarrow \begin{cases}\tau\left(i_{1}, j_{1}\right)<\tau\left(i_{2}, j_{2}\right) & \text { or } \\ \tau\left(i_{1}, j_{1}\right)=\tau\left(i_{2}, j_{2}\right), j_{1}<j_{2} & \text { or } \\ \tau\left(i_{1}, j_{1}\right)=\tau\left(i_{2}, j_{2}\right), j_{1}=j_{2}, i_{1}<i_{2}, & \end{cases}
$$

we obtain

Definition 62. An S-element $b(i, j), 1 \leq i<j \leq u, \mathbf{e}_{l_{i}}=\mathbf{e}_{l_{j}}$, and the related S-pair $\{i, j\}$ are called redundant if either

(a). exists $k>j, \mathbf{e}_{l_{k}}=\mathbf{e}_{l_{i}}=\mathbf{e}_{l_{j}}$ such that

$$
\mu(i, j, k)=\mu(i, j) ; \mu(i, k) \neq \mu(i, j) \neq \mu(j, k)
$$

(b). or exists $k<j, \mathbf{e}_{l_{k}}=\mathbf{e}_{l_{i}}=\mathbf{e}_{l_{j}}: \mu(j, k) \mid \mu(i, j) \neq \mu(k, j)$.

Lemma 63 (Möller). The following holds

1. for each $i, j, k: 1 \leq i, j, k \leq u, \mathbf{e}_{l_{i}}=\mathbf{e}_{l_{j}}=\mathbf{e}_{l_{k}}$, it holds

$$
\frac{\mu(i, j, k)}{\mu(i, k)} b(i, k)-\frac{\mu(i, j, k)}{\mu(i, j)} b(i, j)+\frac{\mu(i, j, k)}{\mu(k, j)} b(k, j)=0 .
$$


2. $\Re:=\left\{b(i, j), 1 \leq i<j \leq u, \mathbf{e}_{l_{i}}=\mathbf{e}_{l_{j}}\right.$ and not redundant $\}$ is a useful S-element set.

3. Let $G:=\left\{g_{1}, \ldots, g_{s}\right\}, s \leq u$, and let

$$
\left(\mathfrak{5} \mathfrak{M}_{*} \subset\left\{\{i, j\}, 1 \leq i<j<s, \mathbf{e}_{l_{i}}=\mathbf{e}_{l_{j}}\right\}\right.
$$

be a useful S-pair set for $G_{*}=\left\{g_{1}, \ldots, g_{s-1}\right\}$.

Let $\bar{M}:=\left\{\mu(j, s): 1 \leq j<s, \mathbf{e}_{l_{j}}=\mathbf{e}_{l_{s}}\right\}$ and let $\bar{M}^{\prime} \subset \bar{M}$ be the set of the elements $\mu:=\mu(j, s) \in \bar{M}$ such that there exists $\mu\left(j^{\prime}, s\right) \in \bar{M}: \mu\left(j^{\prime}, s\right) \mid \mu(j, s) \neq \mu\left(j^{\prime}, s\right)$.

For each $\mu:=\mathbf{M}(j, s) \in \bar{M} \backslash \bar{M}^{\prime}$ let $i_{\mu}, 1 \leq i_{\mu}<s$, be such that $\mu=\mathbf{M}\left(i_{\mu}, s\right)$. Then

$$
\mathfrak{5 M}:=\mathfrak{F M M}_{*} \cup\left\{\left\{i_{\mu}, s\right\}: \mu \in \bar{M} \backslash \bar{M}^{\prime}\right\}
$$

is a useful S-pair set for $G$.

Proof. 1. (cf. [30, Lemma 25.1.4]) One has

$$
\begin{aligned}
& \frac{\mu(i, j, k)}{\mu(i, k)} * b(i, k)-\frac{\mu(i, j, k)}{\mu(i, j)} * b(i, j)+\frac{\mu(i, j, k)}{\mu(k, j)} * b(k, j) \\
= & \frac{\mu(i, j, k)}{\mu(i, k)} *\left(\frac{\mu(i, k)}{\mu(k)} e_{k}-\frac{\mu(i, k)}{\mu(i)} e_{i}\right) \\
- & \frac{\mu(i, j, k)}{\mu(i, j)} *\left(\frac{\mu(i, j)}{\mu(j)} e_{j}-\frac{\mu(i, j)}{\mu(i)} e_{i}\right) \\
+ & \frac{\mu(i, j, k)}{\mu(k, j)} *\left(\frac{\mu(k, j)}{\mu(j)} e_{j}-\frac{\mu(k, j)}{\mu(k)} e_{k}\right) \\
= & \left(\frac{\mu(i, j, k)}{\mu(k)} e_{k}-\frac{\mu(i, j, k)}{\mu(i)} e_{i}\right) \\
- & \left(\frac{\mu(i, j, k)}{\mu(j)} e_{j}-\frac{\mu(i, j, k)}{\mu(i)} e_{i}\right) \\
+ & \left(\frac{\mu(i, j, k)}{\mu(j)} e_{j}-\frac{\mu(i, j, k)}{\mu(k)} e_{k}\right) \\
= & 0
\end{aligned}
$$

2. (cf. [30, Lemma 25.1.8]) In order to prove the claim by induction, it is sufficient to show that, for each redundant $\{i, j\}, 1 \leq i<j \leq u, \mathbf{e}_{l_{i}}=\mathbf{e}_{l_{j}}=: \epsilon$, there are

$-\left\{i_{1}, j_{1}\right\}, \ldots,\left\{i_{\rho}, j_{\rho}\right\}, \ldots,\left\{i_{r}, j_{r}\right\}, 1 \leq i_{\rho}<j_{\rho} \leq u, \mathbf{e}_{i_{\rho}}=\mathbf{e}_{l_{\rho}}=\epsilon$,

- elements $t_{1}, \ldots, t_{r} \in \mathcal{T}$,

- and coefficients $c_{1}, \ldots c_{r} \in R \backslash\{0\}$

such that

- $b(i, j)=\sum_{\rho} c_{\rho} t_{\rho} * b\left(i_{\rho}, j_{\rho}\right)$;

- $\tau(i, j)=t_{\rho} \circ \tau\left(i_{\rho}, j_{\rho}\right)$, for each $\rho$; 
$-\left\{i_{\rho}, j_{\rho}\right\}<\{i, j\}$.

In order to show this, we only need to consider the representation

$$
b(i, j)=\frac{\mu(i, j, k)}{\mu(i, k)} * b(i, k)+\frac{\mu(i, j, k)}{\mu(k, j)} * b(k, j)
$$

and to prove that

$$
\{i, k\}<\{i, j\}>\{k, j\}
$$

this happens (according to the two cases of the definition) because

(a) $\tau(i, k) \mid \tau(i, j, k)=\tau(i, j) \neq \tau(i, k)$ implies $\{i, k\}<\{i, j\}$ and the same argument proves $\{j, k\}<\{i, j\}$;

(b) the same argument as that above proves $\{j, k\}<\{i, j\}$, while $\{i, k\}<\{i, j\}$ because $\tau(i, k) \leq \tau(i, j)$ and $k<j$.

3. (cf. [30, Lemma 25.1.9]) Let $i<s, \mathbf{e}_{l_{i}}=\mathbf{e}_{l_{s}}=: \epsilon, \mu:=\mu(i, s)$. Then:

- if there exists $\mu^{\prime} \in \bar{M}$ such that $\mu\left(i_{\mu^{\prime}}, s\right)=\mu^{\prime} \mid \mu(i, s) \neq \mu^{\prime}$, then since $i_{\mu^{\prime}}<s,\{i, s\}$ is redundant;

- if $i=i_{\mu}$ then $\left\{i_{m}, s\right\} \in \mathfrak{5 M}$;

- if $i \neq i_{\mu}$ then $b(i, s)=\frac{\mu\left(i, i_{\mu}, s\right)}{\mu\left(i, i_{\mu}\right)} b\left(i, i_{\mu}\right)-b\left(i_{\mu}, s\right)$.

Corollary 64. With the present notation, under the assumption that $R$ is a principal ideal domain, $F$ is a Gröbner basis of $\mathrm{M}$ iff each S-polynomial $B(i, j),\{i, j\} \in \mathfrak{R}$ has a Gröbner representation in terms of $F$.

Example 65. In connection with Lemma63 we have

\begin{tabular}{lll|l}
$(i, j, k)$ & $c(i, j, k)$ & $\mu(i, j, k)$ & $b(i, j, k)$ \\
\hline$(1,2,3)$ & $6^{4} \cdot 5 x^{2}$ & $Y_{1}^{2} Y_{2}^{3} Y_{3}^{2}$ & $b(1,2)-2^{2} 3^{3} x b(1,3)+b(2,3)=0$, \\
$(1,2,4)$ & $6^{4} \cdot 5 x^{2}$ & $Y_{1}^{2} Y_{2}^{3} Y_{3}^{2}$ & $b(1,2)-2^{2} 3^{3} x b(1,4)+5 Y_{2} * b(2,4)=0,$. \\
$(1,3,4)$ & $60 x$ & $Y_{1}^{2} Y_{2}^{3} Y_{3}^{2}$ & $b(1,3)-b(1,4)+2 b(3,4)=0$. \\
$(2,3,4)$ & $6^{4} \cdot 5 x^{2}$ & $Y_{1}^{2} Y_{2}^{3} Y_{3}^{2}$ & $b(2,3)-5 Y_{2} * b(2,4)+6^{3} b(3,4)=0$.
\end{tabular}

Note that we obviously [19, 33] have also

$$
b(1,2,3)-b(1,2,4)+2^{2} 3^{3} x b(1,3,4)-b(2,3,4) .
$$

Thus the redundant elements are $b(2,3)$ via 1 or $4, b(1,2)$ via 4 and $b(1,4)$ via 3 .

But, as it is well-known, it is more efficient (if else for storing considerations) the algorithm sketched in Lemma 633 which

for $s=2$ stores $(1,2)$,

for $s=3$ stores $(1,3)$,

for $s=4$ removes $(1,2)$ and stores $(2,4)$ and $(3,4)$. 
Thus the Gebauer Möller set is still

$$
\{b(1,3), b(2,4), b(3,4)\}
$$

while

$$
\begin{aligned}
& b(1,4)=b(1,3)+2 b(3,4), \\
& b(2,3)=5 Y_{2} * b(2,4)+6^{3} b(3,4), \\
& b(1,2)=2^{2} 3^{3} x b(1,4)-5 Y_{2} * b(2,4) .
\end{aligned}
$$

\section{Weispfenning Completions for Bilateral Gröbner ba- sis for Multivariate Ore Extensions of Zacharias Do- mains}

\subsection{Kandri-Rody-Weispfenning completion}

The most efficient technique for producing bilateral Gröbner bases $G:=\mathbb{I}_{2}(F)$ in a noetherian Ore extension is Kandri-Rody-Weispfenning completion [21]. Iteratively:

- Repeat

- Compute a left-Gröbner basis $G$ of the ideal $\mathbb{I}_{L}(F)$;

- for each $g \in G, 1 \leq i \leq n$, compute the normal form $\mathrm{NF}\left(g \star Y_{i}, \mathbb{I}_{L}(F)\right)$ of $g \star Y_{i}$ w.r.t. $G$;

- set $H:=\left\{\mathrm{NF}\left(g \star Y_{i}, \mathbb{I}_{L}(G)\right), g \in G, 1 \leq i \leq n\right\}, F:=G \cup H$

until $H=\emptyset$.

The rationale of the algorithm is

Lemma 66 (Kandri-Rody-Weispfenning). For $G \subset \mathrm{R}$ the following conditions are equivalent:

1. $\mathbb{I}_{L}(G)=\mathbb{I}_{2}(G)$

2. for each $\tau \in \mathcal{T}$ and each $g \in G, g \star \tau \in \mathbb{I}_{L}(G)$;

3. for each $i, 1 \leq i \leq n$, and each $g \in G, g \star Y_{i} \in \mathbb{I}_{L}(G)$.

Proof.

(1) $\Longrightarrow(2) \Longleftrightarrow$ (3) is trivial.

(2) $\Longrightarrow(1) \mathcal{B}_{2}(G):=\{\lambda \star g \star \rho: \lambda, \rho \in \mathcal{T}, g \in G\}$ is an $R$-linear basis of $\mathbb{I}_{2}(G)$ and satisfies

$$
\mathcal{B}_{2}(G)=\{\lambda \star(g \star \rho): \lambda, \rho \in \mathcal{T}, g \in G\} \subseteq\left\{\lambda \star h: \lambda \in \mathcal{T}, h \in \mathbb{I}_{L}(G)\right\} \subseteq \mathbb{I}_{L}(G)
$$




\subsection{Weispfenning: Restricted Representation and Completion}

We can wlog assume that $R$ is effectively given as a quotient $R=\mathcal{R} / \mathrm{I}$ of a free monoid $\operatorname{ring} \mathcal{R}:=\mathbb{Z}\langle\overline{\mathbf{v}}\rangle$ (over $\mathbb{Z}$ and the monoid $\langle\overline{\mathbf{v}}\rangle$ of all words over the alphabet $\overline{\mathbf{v}}$ ) modulo a bilateral ideal I.

We must restrict ourselves to the case in which $<$ is a sequential term-ordering, id est for each $\tau \in \mathcal{T}$, the set $\{\omega \in \mathcal{T}: \omega<\tau\}$ is finite.

Lemma 67. [52] Let

$$
F:=\left\{g_{1}, \ldots, g_{u}\right\} \subset \mathrm{R}^{m}, g_{i}=\mathbf{M}\left(g_{i}\right)-p_{i}=: c_{i} \tau_{i} \mathbf{e}_{\iota_{i}}-p_{i} ;
$$

set $\Omega:=\max _{<}\left\{\mathbf{T}\left(g_{i}\right): 1 \leq i \leq u\right\}$.

Let $\mathrm{M}$ be the bilateral module $\mathrm{M}:=\mathbb{I}_{2}(F)$ and $\mathbb{I}_{W}(F)$ the restricted module

$$
\mathbb{I}_{W}(F):=\operatorname{Span}_{R}(a f \star \rho: a \in R \backslash\{0\}, \rho \in \mathcal{T}, f \in F)
$$

If every $f \star \alpha_{v}(v), f \in F, v \in \overline{\mathbf{v}}, v \in \mathcal{T}, v<\Omega$, has a restricted representation in terms of $F$ w.r.t. a sequential term-ordering $<$, then every $f \star r, f \in F, r \in \mathrm{R}$, has a restricted representation in terms of $F$ w.r.t. $<$.

Proof. We can wlog assume $r=\prod_{i=1}^{v} v_{i}, v_{i} \in \overline{\mathbf{v}}$ and prove the claim by induction on $v \in \mathbb{N}$.

Thus we have a restricted representation in terms of $F$

$$
f \star\left(\prod_{i=1}^{\gamma-1} v_{i}\right)=\sum_{j} d_{j} g_{i_{j}} \star \rho_{j}, \tau_{i_{j}} \circ \rho_{j} \leq \mathbf{T}(f),
$$

whence we obtain

$$
f \star \prod_{i=1}^{v} v_{i}=\left(f \star \prod_{i=1}^{v-1} v_{i}\right) \star v_{v}=\sum_{j} d_{j} g_{i_{j}} \star\left(\rho_{j} \star v_{v}\right)=\sum_{j} d_{j} g_{i_{j}} \star \alpha_{\rho_{j}}\left(v_{v}\right) \rho_{j}
$$

and since $\rho_{j}<\mathbf{T}(f) \leq \Omega$ each element $g_{i_{j}} \star \alpha_{\rho_{j}}\left(v_{v}\right)$ can be substituted with its restricted representation whose existence is granted by assumption.

Lemma 68. [52] Under the same assumption, if, for each $g_{j} \in F$, both $Y_{i} \star g_{j}, 1 \leq i \leq n$ and each $g_{j} \star \alpha_{v}(v), v \in \overline{\mathbf{v}}, v \in \mathcal{T}, v<\Omega$, have a restricted representation in terms of $F$ w.r.t. $<$, then $\mathbb{I}_{W}(F)=$ M.

Proof. It is sufficient to show that, for each $f \in \mathbb{I}_{W}(F)$, both each $Y_{i} \star f \in \mathbb{I}_{W}(F), 1 \leq$ $i \leq n$ and each $f \star r \in \mathbb{I}_{W}(F), r \in \mathrm{R}$.

By assumption $f=\sum_{j} d_{j} g_{i_{j}} \star \rho_{j}, d_{j} \in R \backslash\{0\}, \rho_{j} \in \mathcal{T}, 1 \leq i_{j} \leq u$, so that

$$
Y_{i} \star f=\sum_{j} \alpha_{i}\left(d_{j}\right) \star\left(Y_{i} \star g_{i_{j}}\right) \star \rho_{j} \text { and } f \star r=\sum_{j} d_{j}\left(g_{i_{j}} \star \alpha_{\rho_{j}}(r)\right) \star \rho_{j} ;
$$

by assumption each $Y_{i} \star g_{i_{j}}$ has a restricted representation in terms of $F$; for the Lemma above, also each $g_{i_{j}} \star \alpha_{\rho_{j}}(r)$ has a restricted representation in terms of $F$. 
Corollary 69. [52] Let

$$
F:=\left\{g_{1}, \ldots, g_{u}\right\} \subset \mathrm{R}^{m}, g_{i}=\mathbf{M}\left(g_{i}\right)-p_{i}=: c_{i} \tau_{i} \mathbf{e}_{l_{i}}-p_{i} .
$$

Let $\mathrm{M}$ be the bilateral module $\mathrm{M}:=\mathbb{I}_{2}(F)$ and $\mathbb{I}_{W}(F)$ the restricted module

$$
\mathbb{I}_{W}(F):=\operatorname{Span}_{R}(a f \star \rho: a \in R \backslash\{0\}, \rho \in \mathcal{T}, f \in F) .
$$

$F$ is the bilateral Gröbner basis of $\mathrm{M}$ iff

1. denoting $(5 \mathfrak{M}(F)$ any restricted Gebauer-Möller set for $F$, each $\sigma \in \mathbb{5} \mathfrak{M}(F)$ has a restricted quasi-Gröbner representation in terms of $F$;

2. for each $g_{j} \in F$, both $Y_{i} \star g_{j}, 1 \leq i \leq n$ and each $g_{j} \star \alpha_{v}(v), v \in \overline{\mathbf{v}}, v \in \mathcal{T}, v<\Omega$, have a restricted representation in terms of $F$ w.r.t. $<$.

\subsection{Gebauer-Möller sets for Restricted Gröbner bases}

It is clear from Corollary 69 that the computation of a Gröbner bases can be obtained via Weispfenning's completion, provided that we are able to produce restricted Gebauer-Möller sets; to do so, we need only to properly reformulate the results of Section 7.2

We begin by remarking that for each monomial $c \tau \in \mathrm{M}(\mathrm{R})$ the function $g \mapsto c g \star \tau$ distributes, thus we can define a multiplication $\diamond: R \times R \rightarrow R$ by setting

$$
c_{i} \tau_{i} \diamond c_{j} \tau_{j}:=c_{i} c_{j} \tau_{j} \tau_{i}=c_{j} c_{i} \tau_{i} \tau_{j}=: c_{j} \tau_{j} \diamond c_{i} \tau_{i}
$$

which of course is commutative and thus, granting the trivial syzygy

$$
g_{i} \diamond g_{j}=g_{j} \diamond g_{i}
$$

allows to recover Buchberger First Criterium.

As a consequence, we can define the notion of restricted Gröbner representation:

- we say that $f \in \mathrm{R}^{m} \backslash\{0\}$ has a restricted Gröbner representation in terms of $G$ if it can be written as $f=\sum_{i=1}^{u} l_{i} \diamond g_{i}$, with $l_{i} \in \mathrm{R}, g_{i} \in G$ and $\mathbf{T}\left(l_{i}\right) \circ \mathbf{T}\left(g_{i}\right) \leq$ $\mathbf{T}(f)$ for each $i$.

Let us denote, for each $i, j, 1 \leq i<j \leq u, \mathbf{e}_{l_{i}}=\mathbf{e}_{l_{j}}$,

$$
\begin{aligned}
b_{W}(i, j) & :=\frac{\operatorname{lcm}\left(c_{i}, c_{j}\right)}{c_{j}} e_{j} \frac{\operatorname{lcm}\left(\tau_{i}, \tau_{j}\right)}{\tau_{j}}-\frac{\operatorname{lcm}\left(c_{i}, c_{j}\right)}{c_{i}} e_{i} \frac{\operatorname{lcm}\left(\tau_{i}, \tau_{j}\right)}{\tau_{i}} \\
& =\frac{\mathbf{M}(i, j)}{\mathbf{M}(j)} \diamond e_{j}-\frac{\mathbf{M}(i, j)}{\mathbf{M}(i)} \diamond e_{i} \in \operatorname{ker}\left(\mathfrak{s}_{W}\right), \\
B_{W}(i, j) & :=\frac{\operatorname{lcm}\left(c_{i}, c_{j}\right)}{c_{j}} g_{j} \star \frac{\operatorname{lcm}\left(\tau_{i}, \tau_{j}\right)}{\tau_{j}}-\frac{\operatorname{lcm}\left(c_{i}, c_{j}\right)}{c_{i}} g_{i} \star \frac{\operatorname{lcm}\left(\tau_{i}, \tau_{j}\right)}{\tau_{i}} \\
& =\frac{\mathbf{M}(i, j)}{\mathbf{M}(j)} \diamond g_{j}-\frac{\mathbf{M}(i, j)}{\mathbf{M}(i)} \diamond g_{i} .
\end{aligned}
$$

and let us explicitly assume that 
- for each $g_{j} \in F$, both $Y_{i} \star g_{j}, 1 \leq i \leq n$ and each $g_{j} \star \alpha_{v}(v), v \in \overline{\mathbf{v}}, v \in \mathcal{T}, v<$ $\mathbf{T}\left(g_{j}\right)$, have a restricted representation in terms of $F$ w.r.t. $<$.

Lemma 70 (Buchberger's First Criterion). If $m=1$, id est $F \subset \mathrm{R}$ and $\mathbb{I}_{W}(F)$ is an ideal of $\mathrm{R}$, then

$$
\begin{aligned}
\mathbf{M}(i) \diamond \mathbf{M}(j)=\mathbf{M}(i, j) & \Longleftrightarrow \quad \operatorname{cm}\left(\tau_{i}, \tau_{j}\right)=\tau_{i} \tau_{j} \text { and } \operatorname{lcm}\left(c_{i}, c_{j}\right)=c_{i} c_{j} \\
& \Longrightarrow \quad \mathrm{NF}_{W}\left(B_{W}(i, j), F\right)=0 .
\end{aligned}
$$

Proof. We will prove that $B_{W}(i, j)$ has a restricted Gröbner representation in terms of $F$; thus the result will follow by the equivalence Proposition 19, (4)

Remark that

$$
p_{i}:=g_{i}-\mathbf{M}(i)=\sum_{l} c_{i l} t_{i l} \text { and } p_{j}:=g_{j}-\mathbf{M}(j)=\sum_{k} c_{j k} t_{j k}
$$

satisfy $\mathbf{T}\left(p_{i}\right)<\mathbf{T}\left(g_{i}\right), \mathbf{T}\left(p_{j}\right)<\mathbf{T}\left(g_{j}\right)$.

Then it holds:

$$
0=g_{i} \diamond g_{j}-g_{j} \diamond g_{i}=\mathbf{M}(i) \diamond g_{j}+p_{i} \diamond g_{j}-\mathbf{M}(j) \diamond g_{i}-p_{j} \diamond g_{i},
$$

and

$$
B_{W}(i, j):=\frac{\mathbf{M}(i, j)}{\mathbf{M}(j)} \diamond g_{j}-\frac{\mathbf{M}(i, j)}{\mathbf{M}(i)} \diamond g_{i}=\mathbf{M}(i) \diamond g_{j}-\mathbf{M}(j) \diamond g_{i}=p_{j} \diamond g_{i}-p_{i} \diamond g_{j} .
$$

There are then two possibilities: either

- $\mathbf{M}\left(p_{j}\right) \diamond \mathbf{M}\left(g_{i}\right) \neq \mathbf{M}\left(p_{i}\right) \diamond \mathbf{M}\left(g_{j}\right)$ in which case

$$
\mathbf{T}\left(B_{W}(i, j)\right)=\max \left(\mathbf{T}\left(p_{j}\right) \circ \mathbf{T}\left(g_{i}\right), \mathbf{T}\left(p_{i}\right) \circ \mathbf{T}\left(g_{j}\right)\right)
$$

and

$$
B_{W}(i, j)=p_{j} \diamond g_{i}-p_{i} \diamond g_{j}=\sum_{k} c_{j k} g_{i} \star t_{j k}-\sum_{l} c_{i l} g_{j} \star t_{i l}
$$

is a restricted Gröbner representation;

- or $\mathbf{M}\left(p_{j}\right) \diamond \mathbf{M}\left(g_{i}\right)=\mathbf{M}\left(p_{i}\right) \diamond \mathbf{M}\left(g_{j}\right), \mathbf{T}\left(B_{W}(i, j)\right)<\mathbf{T}\left(p_{j}\right) \circ \mathbf{T}\left(g_{i}\right)=\mathbf{T}\left(p_{i}\right) \circ \mathbf{T}\left(g_{j}\right)$, in which case $B_{W}(i, j)=p_{j} \diamond g_{i}-p_{i} \diamond g_{j}$ would not be a Gröbner representation.

But the latter case is impossible: in fact, from

$$
\mathbf{T}\left(p_{i}\right) \circ \mathbf{T}\left(g_{j}\right)=\mathbf{T}\left(p_{j}\right) \circ \mathbf{T}\left(g_{i}\right)<\mathbf{T}\left(g_{j}\right) \circ \mathbf{T}\left(g_{i}\right)
$$

we deduce $\operatorname{lcm}\left(\mathbf{T}\left(g_{i}\right), \mathbf{T}\left(g_{j}\right)\right) \neq \mathbf{T}\left(g_{j}\right) \circ \mathbf{T}\left(g_{i}\right)$ and $\mathbf{T}(i, j) \neq \mathbf{T}(i) \circ \mathbf{T}(j)$ contradicting the assumption $\mathbf{M}(i, j)=\mathbf{M}(i) \diamond \mathbf{M}(j)$.

Definition 71. Denote

$$
\mathfrak{C}_{u}:= \begin{cases}\{\{i, j\}: \mathbf{M}(i) \diamond \mathbf{M}(j)=\mathbf{M}(i, j)\} & \text { if } \mathbf{M} \text { is an ideal } \\ \emptyset & \text { otherwise. }\end{cases}
$$

A useful S-pair set for $F$ is any subset

$$
\mathfrak{5 M M} \subset \mathcal{S}(u)=\left\{\{i, j\}, 1 \leq i<j \leq u, \mathbf{e}_{l_{i}}=\mathbf{e}_{l_{j}}\right\}
$$

such that $\left\{b(i, j):\{i, j\} \in \mathfrak{G M} \cup \mathfrak{C}_{u}\right\}$ is a Gebauer-Möller set for $F$. 
Corollary 72. With the present notation, under the assumption that $R$ is a principal ideal domain, $F$ is a Gröbner basis of $\mathrm{M}$ iff, denoting $(5 \mathfrak{M}$ a useful $S$-pair set for $F$, each S-polynomial $B_{W}(i, j),\{i, j\} \in \mathfrak{5 M M}$ has a Gröbner representation in terms of $F$.

Proof. By definition $\left\{b_{W}(i, j):\{i, j\} \in \mathfrak{5 M M} \cup \mathfrak{c}_{u}\right\}$ is a Gebauer-Möller set for $F$ so that, by Theorem 33, $F$ is a Gröbner basis of M iff each S-polynomial $B_{W}(i, j),\{i, j\} \in \mathfrak{F} \mathfrak{M} \cup$ $\mathfrak{C}_{u}$ has a Gröbner representation in terms of $F$.

The claim is a direct consequence of Buchberger's First Criterion which states that for each $\{i, j\} \in \mathfrak{C}_{u}, B_{W}(i, j)$ has a weak Gröbner representation in terms of $F$.

Definition 73. An S-element $b(i, j), 1 \leq i<j \leq u, \mathbf{e}_{l_{i}}=\mathbf{e}_{l_{j}}$, and the related S-pair $\{i, j\}$ are called redundant if either

(a). exists $k>j, \mathbf{e}_{l_{k}}=\mathbf{e}_{l_{i}}=\mathbf{e}_{l_{j}}$ such that

$$
\mathbf{M}(i, j, k)=\mathbf{M}(i, j) ; \mathbf{M}(i, k) \neq \mathbf{M}(i, j) \neq \mathbf{M}(j, k),
$$

(b). or exists $k<j, \mathbf{e}_{l_{k}}=\mathbf{e}_{l_{i}}=\mathbf{e}_{l_{j}}: \mathbf{M}(j, k) \mid \mathbf{M}(i, j) \neq \mathbf{M}(j, k)$.

Lemma 74 (Möller). The following holds

1. for each $i, j, k: 1 \leq i, j, k \leq u, \mathbf{e}_{l_{i}}=\mathbf{e}_{l_{j}}=\mathbf{e}_{l_{k}}$, it holds

$$
\frac{c(i, j, k)}{c(i, k)} b(i, k) * \frac{\tau(i, j, k)}{\tau(i, k)}-\frac{c(i, j, k)}{c(i, j)} b(i, j) * \frac{\tau(i, j, k)}{\tau(i, j)}+\frac{c(i, j, k)}{c(k, j)} b(k, j) * \frac{\tau(i, j, k)}{\tau(k, j)}=0 .
$$

2. $\Re:=\left\{b(i, j), 1 \leq i<j \leq u, \mathbf{e}_{l_{i}}=\mathbf{e}_{l_{j}}\right.$ and not redundant $\}$ is a useful $S$-element set.

3. Let $G:=\left\{g_{1}, \ldots, g_{s}\right\}, s \leq u$, and let

$$
\left(\mathfrak{5 M} \mathfrak{M}_{*} \subset\left\{\{i, j\}, 1 \leq i<j<s, \mathbf{e}_{l_{i}}=\mathbf{e}_{l_{j}}\right\}\right.
$$

be a useful $S$-pair set for $G_{*}=\left\{g_{1}, \ldots, g_{s-1}\right\}$.

Let $\bar{M}:=\left\{\mathbf{M}(j, s): 1 \leq j<s, \mathbf{e}_{l_{j}}=\mathbf{e}_{l_{s}}\right\}$ and let $\bar{M}^{\prime} \subset \bar{M}$ be the set of the elements $\mu:=\mathbf{M}(j, s) \in \bar{M}$ such that either

- there exists $\mathbf{M}\left(j^{\prime}, s\right) \in \bar{M}: \mathbf{M}\left(j^{\prime}, s\right) \mid \mathbf{M}(j, s) \neq \mathbf{M}\left(j^{\prime}, s\right)$ or

- (in case $\mathrm{M}$ is an ideal) there exists $i_{\mu}, 1 \leq i_{\mu}<s$ :

$$
\mathbf{M}\left(i_{\mu}\right) \diamond \mathbf{M}(s)=\mathbf{M}\left(i_{\mu}, s\right)=\mu .
$$

For each $\mu:=\mathbf{M}(j, s) \in \bar{M} \backslash \bar{M}^{\prime}$ let $i_{\mu}, 1 \leq i_{\mu}<s$, be such that $\mu=\mathbf{M}\left(i_{\mu}, s\right)$. Then

$$
\mathfrak{5 M}:=\mathfrak{F M M}_{*} \cup\left\{\left\{i_{\mu}, s\right\}: \mu \in \bar{M} \backslash \bar{M}^{\prime}\right\}
$$

is a useful S-pair set for $G$. 
Proof.

1. (cf. [30, Lemma 25.1.4]) One has

$$
\begin{aligned}
& \frac{\mathbf{M}(i, j, k)}{\mathbf{M}(i, k)} \diamond b(i, k)-\frac{\mathbf{M}(i, j, k)}{\mathbf{M}(i, j)} \diamond b(i, j)+\frac{\mathbf{M}(i, j, k)}{\mathbf{M}(k, j)} \diamond b(k, j) \\
= & \frac{\mathbf{M}(i, j, k)}{\mathbf{M}(i, k)} \diamond\left(\frac{\mathbf{M}(i, k)}{\mathbf{M}(k)} \diamond e_{k}-\frac{\mathbf{M}(i, k)}{\mathbf{M}(i)} \diamond e_{i}\right) \\
- & \frac{\mathbf{M}(i, j, k)}{\mathbf{M}(i, j)} \diamond\left(\frac{\mathbf{M}(i, j)}{\mathbf{M}(j)} \diamond e_{j}-\frac{\mathbf{M}(i, j)}{\mathbf{M}(i)} \diamond e_{i}\right) \\
+ & \frac{\mathbf{M}(i, j, k)}{\mathbf{M}(k, j)} \diamond\left(\frac{\mathbf{M}(k, j)}{\mathbf{M}(j)} \diamond e_{j}-\frac{\mathbf{M}(k, j)}{\mathbf{M}(k)} \diamond e_{k}\right) \\
= & \left(\frac{\mathbf{M}(i, j, k)}{\mathbf{M}(k)} \diamond e_{k}-\frac{\mathbf{M}(i, j, k)}{\mathbf{M}(i)} \diamond e_{i}\right) \\
- & \left(\frac{\mathbf{M}(i, j, k)}{\mathbf{M}(j)} \diamond e_{j}-\frac{\mathbf{M}(i, j, k)}{\mathbf{M}(i)} \diamond e_{i}\right) \\
+ & \left(\frac{\mathbf{M}(i, j, k)}{\mathbf{M}(j)} \diamond e_{j}-\frac{\mathbf{M}(i, j, k)}{\mathbf{M}(k)} \diamond e_{k}\right) \\
= & 0
\end{aligned}
$$

2. (cf. [30, Lemma 25.1.8]) In order to prove the claim by induction, it is sufficient to show that, for each redundant $\{i, j\}, 1 \leq i<j \leq u, \mathbf{e}_{l_{i}}=\mathbf{e}_{l_{j}}=: \epsilon$, there are

- $\left\{i_{1}, j_{1}\right\}, \ldots,\left\{i_{\rho}, j_{\rho}\right\}, \ldots,\left\{i_{r}, j_{r}\right\}, 1 \leq i_{\rho}<j_{\rho} \leq u, \mathbf{e}_{i_{\rho}}=\mathbf{e}_{l_{j_{\rho}}}=\epsilon$,

- elements $t_{1}, \ldots, t_{r} \in \mathcal{T}$,

- and coefficients $c_{1}, \ldots c_{r} \in R \backslash\{0\}$

such that

- $b(i, j)=\sum_{\rho} c_{\rho} t_{\rho} \diamond b\left(i_{\rho}, j_{\rho}\right)$;

- $\tau(i, j)=t_{\rho} \circ \tau\left(i_{\rho}, j_{\rho}\right)$, for each $\rho$;

- $\left\{i_{\rho}, j_{\rho}\right\}<\{i, j\}$.

In order to show this, we only need to consider the representation

$$
b(i, j)=\frac{\mathbf{M}(i, j, k)}{\mathbf{M}(i, k)} \diamond b(i, k)-\frac{\mathbf{M}(i, j, k)}{\mathbf{M}(k, j)} \diamond b(k, j)
$$

and to prove that

$$
\{i, k\}<\{i, j\}>\{k, j\}
$$

this happens (according to the two cases of the definition) because

(a) $\tau(i, k) \mid \tau(i, j, k)=\tau(i, j) \neq \tau(i, k)$ implies $\{i, k\} \prec\{i, j\}$ and the same argument proves $\{j, k\}<\{i, j\}$;

(b) the same argument as that above proves $\{j, k\}<\{i, j\}$, while $\{i, k\} \prec\{i, j\}$ because $\tau(i, k) \leq \tau(i, j)$ and $k<j$. 
3. (cf. [30, Lemma 25.1.9]) Let $i<s, \mathbf{e}_{l_{i}}=\mathbf{e}_{l_{s}}=: \epsilon, \mu:=\mathbf{M}(i, s)$. Then:

- if there exists $\mu^{\prime} \in \bar{M}$ such that $\mathbf{M}\left(i_{\mu^{\prime}}, s\right)=\mu^{\prime} \mid \mathbf{M}(i, s) \neq \mu^{\prime}$, then since $i_{\mu^{\prime}}<s,\{i, s\}$ is redundant;

- if $i=i_{\mu}$ and $\mathbf{M}\left(i_{\mu}\right) \diamond \mathbf{M}(s)=\mathbf{M}\left(i_{\mu}, s\right)$, then (M is an ideal) $b_{W}\left(i_{\mu}, s\right) \in \mathfrak{C}_{s}$ so that $B_{W}\left(i_{\mu}, s\right)$ has a restricted Gröbner representation in terms of $G$ by Buchberger's First Criterion;

- if $i=i_{\mu}$ and $\mathbf{M}\left(i_{\mu}\right) \diamond \mathbf{M}(s) \neq \mathbf{M}\left(i_{m}, s\right)$ then $\left\{i_{m}, s\right\} \in(\mathfrak{5 M}$;

- if $i \neq i_{\mu}$ then $b(i, s)=\frac{\mathbf{M}\left(i, i_{\mu}, s\right)}{\diamond} \mathbf{M}\left(i, i_{\mu}\right) b\left(i, i_{\mu}\right)-b\left(i_{\mu}, s\right)$.

Corollary 75. With the present notation, under the assumption that $R$ is a principal ideal domain, $F$ is a restricted Gröbner basis of $\mathrm{M}$ iff

1. each $S$-polynomial $B_{W}(i, j),\{i, j\} \in \Re$, has a restricted Gröbner representation in terms of $F$;

2. for each $g_{j} \in F$, both $Y_{i} \star g_{j}, 1 \leq i \leq n$ and each $g_{j} \star \alpha_{v}(v), v \in \overline{\mathbf{v}}, v \in \mathcal{T}, v<$ $\mathbf{T}\left(g_{j}\right)$, have a restricted representation in terms of $F$ w.r.t. $<$.

\section{Structural Theorem for Multivariate Ore Extensions of Zacharias PIDs}

Theorem 76 (Structural Theorem). Let $R$ be a left Zacharias principal ideal domain, $\mathrm{R}:=R\left[Y_{1}, \ldots, Y_{n}\right]$ a multivariate Ore extension of $R,<$ a term-ordering, $\mathrm{M} \subset \mathrm{R}^{m}$ a left module generated by a basis $F:=\left\{g_{1}, \ldots, g_{u}\right\} \subset \mathrm{M}, \mathbf{M}\left(g_{i}\right)=c_{i} \tau_{i} \mathbf{e}_{i}, C(F)$ a completion of $F, \mathfrak{R}:=\left\{B(i, j), 1 \leq i<j \leq u, \mathbf{e}_{l_{i}}=\mathbf{e}_{l_{j}}\right.$ and not redundant $\}$.

Then the following conditions are equivalent:

(1). F is a left Gröbner basis of $\mathrm{M}$;

$\left(1_{s}\right) . C(F)$ is a left strong Gröbner basis of $\mathrm{M}$;

(2). $\mathcal{B}(F):=\{\lambda g: \lambda \in \mathcal{T}, g \in F\}$ is a Gauss generating set [30, Definition 21.2.1];

(3). $f \in \mathrm{M} \Longleftrightarrow$ it has a left Gröbner representation in terms of $F$;

(4). $f \in \mathrm{M} \Longleftrightarrow$ it has a left strong Gröbner representation in terms of $C(F)$;

(5). for each $f \in \mathrm{R}^{m} \backslash\{0\}$ and any normal form $h$ of $f$ w.r.t. $F$, we have

$$
f \in \mathrm{M} \Longleftrightarrow h=0
$$

(5s). for each $f \in \mathrm{R}^{m} \backslash\{0\}$ and any strong normal form $h$ of $f$ w.r.t. $C(F)$, we have

$$
f \in \mathrm{M} \Longleftrightarrow h=0
$$


(6). for each $f \in \mathrm{R}^{m} \backslash\{0\}, f-\operatorname{Can}(f, \mathrm{M})$ has a strong Gröbner representation in terms of $C(F)$;

(7). each $B(i, j) \in \Re$ has a weak Gröbner representation in terms of $F$;

(8). for each element $\sigma$ of a Gebauer-Möller set for $F$, the S-polynomial $\Im_{L}(\sigma)$ has a left quasi-Gröbner representation in terms of $F$.

Proof.

$(1) \Longleftrightarrow\left(1_{s}\right)$ is Proposition 56

(1) $\Longleftrightarrow$ (2) is trivial;

(1) $\Longleftrightarrow(5) \Longleftrightarrow$ (3) is Proposition 19 ,

$\left(1_{s}\right) \Longleftrightarrow(4) \Longleftrightarrow\left(5_{s}\right)$ is Proposition 19 ,

$(1) \Longrightarrow(6)$ is the content of Section 6.3,

(6) $\Longrightarrow$ (4) because for each $f \in \mathrm{M}, \operatorname{Can}(f, \mathrm{M})=0$;

(1) $\Longleftrightarrow(7)$ is Corollary 51

(1) $\Longleftrightarrow(3) \Longleftrightarrow(8)$ is Theorem 33

\section{Spear's Theorem}

For Gröbner bases in a ring $\mathcal{A}$ given as quotient

$$
\Pi: Q:=\mathbb{Z}\left\langle Y_{1}, \ldots, Y_{n}\right\rangle \rightarrow \mathcal{A} \cong Q / \mathcal{I}, \quad I:=\operatorname{ker}(\Pi)
$$

of a free assocative algebra, a general approach is to directly apply Spear's Theorem [48] [30, Proposition 24.7.3] [28], which, while not a tool for computation, can be helpful in order to understand the structure of $\mathcal{A}$.

For the present setting, denoting

- $f_{i j}:=Y_{j} Y_{i}-\alpha_{j}\left(Y_{i}\right) Y_{j}-\delta_{j}\left(Y_{i}\right), 1 \leq i<j \leq n$,

- $C:=\left\{f_{i j}: 1 \leq i<j \leq n\right\}$;

$-\mathcal{I}:=\mathbb{I}_{2}(C)$

and for each $m \in \mathbb{N}$,

- $\left\{\mathbf{e}_{1}, \ldots, \mathbf{e}_{m}\right\}$ the canonical basis of $\mathrm{R}^{m}$,

$-C^{(m)}:=\left\{f_{i j \iota}:=f_{i j} \mathbf{e}_{\iota}: 1 \leq i<j \leq n, 1 \leq \iota \leq m\right\}$, 
we have the presentation

$$
\mathrm{R}=Q / \mathcal{I}, \mathcal{I}:=\operatorname{ker}(\Pi), \Pi: Q:=R\left\langle Y_{1}, \ldots, Y_{n}\right\rangle \rightarrow \mathrm{R}
$$

and, for each free R-module $\mathbf{R}^{m}, m \in \mathbb{N}$, the projection $\Pi$ extends to the canonical projections, still denoted $\Pi$,

$$
\Pi: Q^{m} \rightarrow \mathrm{R}^{m}, \operatorname{ker}(\Pi)=\mathcal{I}^{m}=\mathbb{I}_{2}\left(C^{(m)}\right) .
$$

Thus denoting

- $F:=\left\{g_{1}, \ldots, g_{u}\right\} \subset \mathrm{R}^{m}, g_{i}=\mathbf{M}\left(g_{i}\right)-p_{i}=: c_{i} \tau_{i} \mathbf{e}_{l_{i}}-p_{i}$,

- $\mathrm{M} \subset \mathrm{R}^{m}$ the module $\mathrm{M}:=\mathbb{I}_{2}(F)$,

$-\mathrm{M}^{\prime}:=\Pi^{-1}(\mathrm{M})=\mathrm{M}+\mathcal{I}^{m} \subset Q^{m}$,

we can reformulate Spear's result as

Lemma 77. [28. Lemma 12] Assume $F \subset \mathrm{M}^{\prime}$ is a Gröbner basis of $\mathrm{M}^{\prime}$ and denote

$$
\bar{F}:=\left\{\operatorname{Can}\left(g, \mathcal{I}^{m}\right): g \in F, \mathbf{T}(g) \in \mathcal{T}^{(m)}\right\} \subset R\left[Y_{1}, \ldots, Y_{n}\right]^{m}
$$

where $\operatorname{Can}\left(g, \mathcal{I}^{m}\right)$ denotes the canonical form of $g \in Q^{m}$ w.r.t. $C^{(m)}$ so that in particular $g=\Pi(g)$ for each $g \in \bar{F}$.

Then $\bar{F} \sqcup C^{(m)}$ is a Gröbner basis of $\mathrm{M}^{\prime}$.

Theorem 78 (Spear). [28 Theorem 13] With the present notation, the following holds:

1. if $F$ is a reduced Gröbner basis of $\mathrm{M}^{\prime}$, then

$$
\begin{aligned}
\{g \in F: g=\Pi(g)\} & =\left\{\Pi(g): g \in F, \mathbf{T}(g) \in \mathcal{T}^{(m)}\right\} \\
& =F \cap R\left[Y_{1}, \ldots, Y_{n}\right]^{m}
\end{aligned}
$$

is a reduced Gröbner basis of $\mathrm{M}$;

2. if $F \subset R\left[Y_{1}, \ldots, Y_{n}\right]^{m}$, so that in particular $\Pi(f)=f$ for each $f \in F$, is the Gröbner basis of $\mathrm{M}$, then $F \sqcup C^{(m)}$ is a Gröbner basis of $\mathrm{M}^{\prime}$.

3. Assume each $m^{\prime} \in \mathrm{M}^{\prime}$ has a Gröbner representation in terms of $F \subset \mathrm{M}^{\prime}$.

Set

$$
\bar{F}:=\left\{\operatorname{Can}\left(g, I^{m}\right): g \in F, g \notin I^{m}\right\} \subset R\left[Y_{1}, \ldots, Y_{n}\right]^{m}
$$

where $\operatorname{Can}\left(g, I^{m}\right) \in R\left[Y_{1}, \ldots, Y_{n}\right]^{m}$ denotes the canonical form of $g \in Q^{m}$ w.r.t. $C^{(m)}$ so that in particular $g=\Pi(g)$ for each $g \in \bar{F}$.

Then each $m \in \mathrm{M}$ has a Gröbner representation in terms of $\bar{F}$.

4. if $F \subset R\left[Y_{1}, \ldots, Y_{n}\right]^{m}$, so that in particular $\Pi(f)=f$ for each $f \in F$, is such that each $m \in \mathrm{M}$ has a Gröbner representation in terms of $F$, then each $m^{\prime} \in \mathrm{M}^{\prime}$ has a Gröbner representation in terms of $F \sqcup C^{(m)}$. 
Corollary 79. [28. Corollary 14] With the present notation and considering

- the bilateral $\mathrm{R}$-module $\left(\mathrm{R} \otimes_{\hat{R}} \mathrm{R}^{\mathrm{op}}\right)^{u}$ with canonical basis $\left\{e_{1}, \ldots, e_{u}\right\}$

- the bilateral $Q$-module $\left(Q \otimes_{\hat{R}} Q^{\mathrm{op}}\right)^{|F|+m|G|}$ with canonical basis

$$
\left\{e_{1}, \ldots, e_{u}\right\} \sqcup\left\{\mathrm{e}_{i j l}: 1 \leq i<j \leq n, 1 \leq \iota \leq m\right\},
$$

- the projections $\Im_{2}:\left(\mathrm{R} \otimes_{\hat{R}} \mathrm{R}^{\mathrm{op}}\right)^{|F|} \rightarrow \mathrm{R}^{m}: \Im_{2}\left(e_{i}\right)=g_{i}, 1 \leq i \leq u$, and

- $\hat{\mathfrak{S}}_{2}:\left(Q \otimes_{\hat{R}} Q^{\mathrm{op}}\right)^{|F|+m|C|} \rightarrow Q^{m}:$

$$
\hat{\Xi}_{2}\left(e_{i}\right)=g_{i}, 1 \leq i \leq u, \quad \hat{\Xi}_{2}\left(\mathrm{e}_{i j l}\right)=f_{i j \iota}, 1 \leq i<j \leq n, 1 \leq \iota \leq m,
$$

- the map

$$
\bar{\Pi}:\left(Q \otimes_{\hat{R}} Q^{\mathrm{op}}\right)^{|F|+m|C|} \rightarrow\left(\mathrm{R} \otimes_{\hat{R}} \mathrm{R}^{\mathrm{op}}\right)^{|F|}
$$

(where each $\lambda, \rho \in R\left\langle Y_{1}, \ldots, Y_{n}\right\rangle, a, b \in R \backslash\{0\}$ )

$$
\bar{\Pi}\left(\sum_{i} a_{i} \lambda_{i} e_{i} b_{i} \rho_{i}+\sum_{i j \iota} a_{i j \iota} \lambda_{i j l} \mathrm{e}_{i j \iota} b_{i j l} \rho_{i j l}\right)=\sum_{i} a_{i} \Pi\left(\lambda_{i}\right) e_{i} b_{i} \Pi\left(\rho_{i}\right),
$$

if $\Sigma \subset\left(Q \otimes_{\hat{R}} Q^{\mathrm{op}}\right)^{|F|+m|C|}$ is a bilateral standard basis of $\operatorname{ker}\left(\hat{\Theta}_{2}\right)$, then $\bar{\Pi}(\Sigma)$ is a bilateral standard basis of $\operatorname{ker}\left(\subseteq_{2}\right)$.

\section{Lazard Structural Theorem for Ore Extensions over a Principal Ideal Domain}

Let $\mathbb{D}$ be a commutative principal ideal domain, $\mathrm{R}:=\mathbb{D}[Y ; \alpha, \delta]$ be an Ore extension and $\mathrm{I} \subset \mathrm{R}$ be a bilateral ideal.

Let $F:=\left\{f_{0}, f_{1}, \ldots, f_{k}\right\}$ be a reduced minimal strong bilateral Gröbner basis of I ordered so that

$$
\operatorname{deg}\left(f_{0}\right) \leq \operatorname{deg}\left(f_{1}\right) \leq \cdots \leq \operatorname{deg}\left(f_{k}\right)
$$

and let us denote, for each $i, c_{i}:=\operatorname{lc}\left(f_{i}\right), r_{i} \in \mathbb{D} \backslash\{0\}$ and $p_{i} \in \mathrm{R}$ the conten 3 and the primitive of $f_{i}$ so that $f_{i}=r_{i} p_{i}$; denoting $P:=p_{0}$ the primitive part of $f_{0}$ and $G_{k+1}:=r_{k} \in \mathbb{D} \backslash\{0\}$ the content of $f_{k}$ we have

Theorem 80. With the present notation, for each $i, 0 \leq i<k$, there is $H_{i+1} \in \mathrm{R}, d(i):=$ $\operatorname{deg}\left(H_{i}\right)$ and $G_{i} \in \mathbb{D} \backslash\{0\}$ such that

- $f_{0}=G_{1} \cdots G_{k+1} P$,

- $f_{j}=G_{j+1} \cdots G_{k+1} H_{j} P, 1 \leq j \leq k$,

\footnotetext{
${ }^{3}$ Defined here as the greatest common divisor of the coefficients of $f_{i}$ in the principal ideal domain $\mathbb{D}$.
} 
and

1. $0<d(1)<d(2)<\cdots<d(k)$;

2. $G_{i} \in \mathbb{D}, 1 \leq i \leq k+1$, is such that $c_{i-1}=G_{i} c_{i}$

3. $P=p_{0}$ (the primitive part of $f_{0} \in \mathrm{R}$ );

4. $H_{i} \in \mathrm{R}$ is a monic polynomial of degree $d(i)$, for each $i$;

5. $H_{i+1} \in\left(G_{1} \cdots G_{i}, G_{2} \cdots G_{i} H_{1}, \ldots, G_{j+1} \cdots G_{i} H_{j}, \ldots, H_{i-1} G_{i}, H_{i}\right)$ for all $i$.

6. $r_{i}=G_{i+1} \cdots G_{k}$.

Proof. Let $P$ and $G_{k+1}$ be, resp., the greatest common right divisor of $\left\{p_{0}, \ldots, p_{k}\right\}$ in $\mathrm{R}$ and the greatest common divisor of $\left\{r_{0}, \ldots, r_{k}\right\}$ in $\mathbb{D}$; since a set $\left\{g_{0}, \ldots, g_{k}\right\}$ is a minimal strong Gröbner basis if and only if the same is true for $\left\{r g_{0} g, \ldots, r g_{k} g\right\}, r \in \mathbb{D}, g \in \mathrm{R}$, we can left divide by $G_{k+1}$ and right divide by $P$ and assume wlog that $P=G_{k+1}=1$ and that both the greatest common right divisor of $\left\{p_{0}, \ldots, p_{k}\right\}$ and the greatest common divisor of $\left\{r_{0}, \ldots, r_{k}\right\}$ are 1 .

Setting $\mathrm{d}(i):=\operatorname{deg}\left(f_{i}\right)$ and $v(i):=\mathrm{d}(i+1)-\mathrm{d}(i)$ for each $i$, by assumption we have $\mathrm{d}(i) \leq \mathrm{d}(i+1)$.

If $\mathrm{d}(i)=\mathrm{d}(i+1)$, let us define

$$
h:=b_{i} f_{i}+b_{i+1} f_{i+1} \in \mathrm{I}
$$

where $c, b_{i}, b_{i+1} \in \mathbb{D}$ are such that $b_{i} c_{i}+b_{i+1} c_{i+1}=c, c$ being the greatest common divisor of $c_{i}$ and $c_{i+1}$, so that $c Y^{\mathrm{d}(i+1)}=\mathbf{M}(h) \in \mathbf{M}(\mathrm{I})$; this implies the existence of $j$ such that $\mathbf{M}\left(f_{j}\right)|\mathbf{M}(h)| \mathbf{M}\left(f_{i+1}\right)$ contradicting minimality; thus $\mathrm{d}(i)<\mathrm{d}(i+1)$ and this, in turn, implies (1) since $d(i)=\mathrm{d}(i)-\operatorname{deg}(P)$.

Both $f_{i} Y^{\gamma(i)}$ and $f_{i+1}$ are in the ideal and have degree $\mathrm{d}(i+1)$.

Therefore, for $c, b_{i}, b_{i+1} \in \mathbb{D}$ such that $b_{i} c_{i}+b_{i+1} c_{i+1}=c, c$ being the greatest common divisor of $c_{i}$ and $c_{i+1}, h:=b_{i} f_{i} Y^{\mathrm{d}(i+1)-\mathrm{d}(i)}+b_{i+1} f_{i+1} \in \mathrm{I}$, so that $c Y^{\mathrm{d}(i+1)}=$ $\mathbf{M}(h) \in \mathbf{M}(\mathrm{I})$ and $\mathbf{M}\left(f_{j}\right) \mid \mathbf{M}(h)$ for some $j$. If $c_{i+1} \neq c$, necessarily $\operatorname{deg}\left(f_{j}\right)<\operatorname{deg}\left(f_{i+1}\right)$ whence $j<i+1$ and $\mathbf{M}\left(f_{j}\right)|\mathbf{M}(h)| \mathbf{M}\left(f_{i+1}\right)$ getting a contradiction. As a conclusion $c_{i}=G_{i+1} c_{i+1}$, for some $G_{i+1} \in \mathbb{D}$ and (2).

Since $G_{i+1} f_{i+1}-f_{i} Y^{v(i)}$ is a polynomial of degree less than $\mathrm{d}(i+1)$ which reduces to zero by the Gröbner basis, it follows that

$$
G_{i+1} f_{i+1} \in \mathbb{I}\left(f_{0}, \ldots, f_{i}\right) \text { for each } i, 0 \leq i<k ;
$$

thus, inductively we obtain

$$
\left.p_{0}\right|_{R} f_{j} \text { for each } j \leq\left. i \Longrightarrow p_{0}\right|_{R} f_{j} \text { for each } j \leq i+1 .
$$

Also

$$
\begin{aligned}
\left.c_{i}\right|_{L} f_{j} \text { for each } j \leq i & \Longrightarrow G_{i+1} c_{i+1}=\left.c_{i}\right|_{L} G_{i+1} f_{i+1} \\
& \left.\Longrightarrow \quad c_{i+1}\right|_{L} f_{j} \text { for each } j \leq i+1 .
\end{aligned}
$$


Therefore, the assumptions that the greatest common right divisor of $\left\{p_{0}, \ldots, p_{k}\right\}$ and the greatest common divisor of $\left\{r_{0}, \ldots, r_{k}\right\}$ are 1 imply that $p_{0}=c_{k}=1$ proving (3); thus in particular $f_{0}=c_{0}$ so that $c_{0} \mid f_{0}$ and this is sufficient to deduce, by the inductive argument, that each $c_{i}$ left-divides $f_{i}$ and therefore coincides with $r_{i}$.

Inductively we obtain

$$
r_{i} \operatorname{lc}(P)=c_{i}=G_{i+1} c_{i+1}=G_{i+1} r_{i+1} \operatorname{lc}(P)=G_{i+1} \cdots G_{k} \operatorname{lc}(P)
$$

thus proving (6); defining $H_{i}$ the polynomial s.t. $c_{i} H_{i} P=f_{i}$ for all $i$ we have $\operatorname{lc}\left(H_{i}\right)=1$ (proving (4)), $d(i)+\operatorname{deg}(P)=\operatorname{deg}\left(f_{i}\right)$ and $G_{i+1} f_{i+1} \in\left(f_{0}, \ldots, f_{i}\right)$ which proves (5) dividing out $G_{i+1} \cdots G_{k}$.

\section{A The PIR case}

While an understandable timor restrained us to violate Ore's tabu requiring degree preservation of product, it is well-known that Zacharias-Möller results are naturally stated for polynomials over PIRs and the restriction to PIDs is unnatural; we therefore sketch here the few modifications to the theory which are required in order to adapt it to Ore extensions R over a PIR $R$.

The first delicate adaptation is required by formula (4]; the natural solution is due to Gateva [15, 16, 17] which considered valuation over the semigroup with zero $\mathcal{T} \cup\{o\}$ instead of $\mathcal{T}$ setting

$$
\circ: \mathbf{N} \times \mathbf{N} \rightarrow \mathbf{N} \cup\{o\}: u \circ v= \begin{cases}\mathbf{T}(u \star v) & u \star v \neq 0 \\ o & u \star v=0 .\end{cases}
$$

Her theory however apply only to domains.

Thus in order to extend Corollary 15 we need to reformulate it as

Corollary 15. If $<$ is a term ordering on $\mathcal{T}$ and $<$ is a $<$-compatible term ordering on $\mathcal{T}^{(m)}$, then, for each $l, r \in \mathrm{R}$ and $f \in \mathrm{R}^{(m)}$,

1. $\mathbf{M}(l \star f)=\mathbf{M}(\mathbf{M}(l) \star \mathbf{M}(f))$ provided $\operatorname{lc}(l) \operatorname{lc}(f) \neq 0$;

2. $\mathbf{M}(f \star r)=\mathbf{M}(\mathbf{M}(f) \star \mathbf{M}(r))$ provided $\operatorname{lc}(f) \operatorname{lc}(r) \neq 0$;

3. $\mathbf{M}(l \star f \star r)=\mathbf{M}(\mathbf{M}(l) \star \mathbf{M}(f) \star \mathbf{M}(r))$ provided $\operatorname{lc}(l) \operatorname{lc}(f) \operatorname{lc}(r) \neq 0$.

4. $\mathbf{T}(l \star f) \leq \mathbf{T}(l) \circ \mathbf{T}(f)$ equality holding provided that $\operatorname{lc}(l) \operatorname{lc}(f) \neq 0$;

5. $\mathbf{T}(f \star r) \leq \mathbf{T}(f) \circ \mathbf{T}(r)$ equality holding provided that $\operatorname{lc}(f) \operatorname{lc}(r) \neq 0$;

6. $\mathbf{T}(l \star f \star r) \leq \mathbf{T}(l) \circ \mathbf{T}(f) \circ \mathbf{T}(r)$ equality holding provided that $\operatorname{lc}(l) \operatorname{lc}(f) \operatorname{lc}(r) \neq 0$.

If, moreover, $R$ is a domain, then

7. $\mathbf{T}(l \star f)=\mathbf{T}(l) \circ \mathbf{T}(f)$;

8. $\mathbf{T}(f \star r)=\mathbf{T}(f) \circ \mathbf{T}(r)$; 
9. $\mathbf{T}(l \star f \star r)=\mathbf{T}(l) \circ \mathbf{T}(f) \circ \mathbf{T}(r)$.

As regards Gröbner basis computation we remark that the first and the third algorithms (Section 6.1 and 6.3 ) apply verbatim also in the PIR case; in the algorithm in fact we have $\{i\} \in \mathfrak{H}(F)$ for each $i, 1 \leq i \leq u$ and thus each $\mathrm{m}:=\mathbf{T}\left(g_{i}\right) \in \mathrm{H}$ is treated by the algorithm which (if the basis is minimal) produces also the annihilitator syzygy

$$
\left.\left(d_{1}, \ldots, d_{u}\right)\right) \in \operatorname{Syz}_{L}\left(v(\mathrm{~m})_{1}, \ldots, v(\mathrm{~m})_{u}\right), d_{j}:= \begin{cases}a_{j} & \text { if } j=i \\ 0 & \text { otherwise }\end{cases}
$$

where we denote, for each $i \leq u, a_{i} \in R$ the annihilator of $\mathbb{I}\left(c_{i}\right)$.

In the second algorithm (Section 6.2) the inductive seed becomes

$$
\mathcal{S}_{1}=\left\{b \in R: b \operatorname{lc}\left(g_{1}\right)=0\right\}=\mathbb{I}\left(a_{1}\right) \subset R,
$$

and, for each $s, 1<s \leq u,\{s\}$ is basic for $\mathbf{T}\left(g_{s}\right)$ provided the basis is minimal.

Therefore

Corollary 49. Assuming that the Zacharias ring $R$ is principal and denoting, for each $i, j, 1 \leq i<j \leq u, \mathbf{e}_{l_{i}}=\mathbf{e}_{l_{j}}$,

$$
\begin{aligned}
b(i, j) & :=\frac{\operatorname{lcm}\left(\alpha_{\{i, j\}, i}\left(c_{i}\right), \alpha_{\{i, j\}, j}\left(c_{j}\right)\right)}{\alpha_{\{i, j\}, j}\left(c_{j}\right)} \frac{\operatorname{lcm}\left(\tau_{i}, \tau_{j}\right)}{\tau_{j}} e_{j} \\
& -\quad \frac{\operatorname{lcm}\left(\alpha_{\{i, j\}, i}\left(c_{i}\right), \alpha_{\{i, j\}, j}\left(c_{j}\right)\right)}{\alpha_{\{i, j\}, i}\left(c_{i}\right)} \frac{\operatorname{lcm}\left(\tau_{i}, \tau_{j}\right)}{\tau_{i}} e_{i}, \\
B(i, j) & :=\frac{\operatorname{lcm}\left(\alpha_{\{i, j\}, i}\left(c_{i}\right), \alpha_{\{i, j\}, j}\left(c_{j}\right)\right)}{\alpha_{\{i, j\}, j}\left(c_{j}\right)} \frac{\operatorname{lcm}\left(\tau_{i}, \tau_{j}\right)}{\tau_{j}} \star g_{j} \\
a(i) & :=a_{i} e_{i} \\
A(i) & :=a_{i} \star g_{i},
\end{aligned}
$$

we have that

$$
\left\{b(i, j): 1 \leq i<j \leq u, \mathbf{e}_{l_{i}}=\mathbf{e}_{l_{j}}\right\} \cup\{a(i), i \leq u\}
$$

is a Gebauer-Möller set for $F$, so that $F$ is a Gröbner basis of $\mathrm{M}$, iff each $B(i, j)$, $1 \leq i<j \leq u, \mathbf{e}_{l_{i}}=\mathbf{e}_{l_{j}}$, and each $A(i), i \leq u$, has a weak Gröbner representation in terms of $F$.

Corollary 51. Assuming that the Zacharias ring $R$ is principal and that each $\alpha_{i}$ is an automorphism denoting, for each $i, j, 1 \leq i<j \leq u, \mathbf{e}_{l_{i}}=\mathbf{e}_{l_{j}}$,

$$
\begin{aligned}
b(i, j) & :=e_{j} \alpha_{\tau_{j}}^{-1}\left(\frac{\operatorname{lcm}\left(c_{i}, c_{j}\right)}{c_{j}}\right) \frac{\operatorname{lcm}\left(\tau_{i}, \tau_{j}\right)}{\tau_{j}}-e_{i} \alpha_{\tau_{i}}^{-1}\left(\frac{\operatorname{lcm}\left(c_{i}, c_{j}\right)}{c_{i}}\right) \frac{\operatorname{lcm}\left(\tau_{i}, \tau_{j}\right)}{\tau_{i}} \\
B(i, j) & :=g_{j} \star \alpha_{\tau_{j}}^{-1}\left(\frac{\operatorname{lcm}\left(c_{i}, c_{j}\right)}{c_{j}}\right) \frac{\operatorname{lcm}\left(\tau_{i}, \tau_{j}\right)}{\tau_{j}}-g_{i} \star \alpha_{\tau_{i}}^{-1}\left(\frac{\operatorname{lcm}\left(c_{i}, c_{j}\right)}{c_{i}}\right) \frac{\operatorname{lcm}\left(\tau_{i}, \tau_{j}\right)}{\tau_{i}} \\
a(i) & :=e_{i} \alpha_{\tau_{i}}^{-1}\left(a_{i}\right), \\
A(i) & :=g_{i} \star \alpha_{\tau_{i}}^{-1}\left(a_{i}\right),
\end{aligned}
$$


we have that

$$
\left\{b(i, j): 1 \leq i<j \leq u, \mathbf{e}_{l_{i}}=\mathbf{e}_{l_{j}}\right\} \cup\{a(i), i \leq u\}
$$

is a Gebauer-Möller set for $F$, so that $F$ is a right Gröbner basis of $\mathrm{M}$, iff each $B(i, j)$, $1 \leq i<j \leq u, \mathbf{e}_{l_{i}}=\mathbf{e}_{l_{j}}$, and each $A(i), i \leq u$, has a right weak Gröbner representation in terms of $F$.

Corollary 58. With the present notation, under the assumption that $R$ is a principal ideal ring, $F$ is a Gröbner basis of I iff, denoting $(5 \mathfrak{M}$ a useful $S$-pair set for $F$, each $S$ polynomial $B(i, j),\{i, j\} \in(\mathfrak{F M}$, and each $A(i), 1 \leq i \leq u$, has a Gröbner representation in terms of $F$.

Corollary 61. With the present notation, under the assumption that $R$ is a principal ideal domain, $F$ is a Gröbner basis of $\mathrm{M}$ iff each $S$-polynomial $B(i, j),\{i, j\} \in \mathfrak{R}$, and each $A(i), 1 \leq i \leq u$, has a Gröbner representation in terms of $F$.

Corollary 62. With the present notation, under the assumption that $R$ is a principal ideal ring, $F$ is a Gröbner basis of $\mathrm{M}$ iff

1.

2. each S-polynomial $B_{W}(i, j),\{i, j\} \in \Re$, and each $A(i), 1 \leq i \leq u$, has a restricted Gröbner representation in terms of $F$;

3. for each $g_{j} \in F$, both $Y_{i} \star g_{j}, 1 \leq i \leq n$ and each $g_{j} \star \alpha_{v}(v), v \in \overline{\mathbf{v}}, v \in \mathcal{T}, v<$ $\mathbf{T}\left(g_{j}\right)$, have a restricted representation in terms of $F$ w.r.t. $<$.

Finally we remark that a Lazard Structural Theorem for Ore Extensions over a Principal Ideal Domain can be easily obtained by adapting the result given by NortonSălăgean [35, 36], [30, § 33.3] for polynomial rings.

\section{References}

[1] Apel J., Gröbnerbasen in Nichetkommutativen Algebren und ihre Anwendung, Dissertation, Leipzig (1988)

[2] Apel J., Computational ideal theory in finitely generated extension rings, Theor. Comp. Sci. 224 (2000), 1-33

[3] Apel J., Lassner, W., An Algorithm for calculations in enveloping fields of Lie algebras, In: Proc. Int. Conf. on Comp. Algebra and its Appl. $n$ Theoretical Physics JINR D11-85-792, Dubna (1985) 231-241

[4] Apel J., Lassner, W., Computation and Simplification in Lie fields, L. N. Comp. Sci. 378 (1987), 468-478, Springer

[5] Bergman G.H., The Diamond Lemma for Ring Theory, Adv. Math. 29 (1978), $178-218$ 
[6] E. Byerne, Gröbner bases over commutative rings and Applications to coding theory in M. Sala et al. (Ed.) Gröbner bases, Coding, Cryptography, Springer Risc XVI, (2009). 239-262

[7] Buchberger B., Ein Algorithmus zum Auffinden der Basiselemente des Restklassenringes nach einem nulldimensionalen Polynomideal, $\mathrm{Ph}$. D. Thesis, Innsbruck (1965)

[8] Buchberger B., Ein algorithmisches Kriterium für die Lösbarkeit eines algebraischen Gleischunssystem, Aeq. Math. 4 (1970), 374-383

[9] Buchberger B., A Critorion for Detecting Unnecessary Reduction in the Construction of Gröbner bases, L. N. Comp. Sci 72 (1979), 3-21, Springer

[10] Buchberger B., Gröbner Bases: An Algorithmic Method in Polynomial Ideal Theory, in Bose N.K. (Ed.) Multidimensional Systems Theory (1985), 184-232, Reider

[11] J. Bueso, J. Gomez-Torrecillas, and A. Verschoren. Methods in Non-Commutative Algebra (2003). Kluwer

[12] Chyzak F., Salvy B. Non-commutative Elimination in Ore Algebras Proves multivariate Identities J. Symb. Comp. 26 (1998), 187-227

[13] Cohn P.M., Noncommutative unique factorization domains Trans. A.M.S. 109 (1963), 313-331

[14] Cohn P.M., Ring with a week Algorithm Trans. A.M.S. 109 (1963), 332-356

[15] Gateva-Ivanova T., Groebner bases in skew polynomial rings, J. Algebra 138 (1991) 13-35

[16] Gateva-Ivanova T., Noetherian Properties of Skew Polynomial Rings with Binomial Relations, Trans. A.M.S. 345 (1994), 203-219,

[17] Gateva-Ivanova T., Skew polynomial rings with binomial relations, J. Algebra 185 (1996) 710-753

[18] Gebauer R., Möller H.M., On an Installation of Buchbgerger's Algorithm, J. Symb. Comp. 6, (1988), 275-286

[19] Janet M. , Sur les systèmes d'équations aux dérivées partielles J. Math. Pure et Appl., 3 (1920), 65-151

[20] Kandri-Rody A., Kapur, D. Computing the Gröbner basis of an ideal in polynomail rings over a Euclidean ring J. Symb. Comp. 6 (1990), 37-56

[21] Kandri-Rody, A., Weispfenning, W., Non-commutativer Gröbner Bases in Algebras of Solvable Type, J. Symb. Comp. 9 (1990), 1-26

[22] Kredel, H. Solvable Polynomial rings Dissertation, Passau (1992) 
[23] LaScala R., Levandovskyy V. Skew Polynomial Rings, Gröbner bases and the Letterplace embedding of the free Associativ algebra, J. Symb. Comp. 48 (2013), $110-131$

[24] Lazard D., Solving zero-dimensional algebraic systems J. Symb. Comp. 15 (1992), 117-132

[25] Levandovskyy V. G., Non-commutative Computer Algebra for Polynomial Algebras: Gröbner Bases, Applications and ImplementationDissertation, Kaiserslautern (2005)

http://kluedo.ub.uni-kl.de/volltexte/2005/1883/

[26] Levandovskyy V. G., PBW Bases, Non-Degeneracy Conditions and Applications In: Buchweitz, R.-O., Lenzing, H. (Eds.), Representation of Algebras and Related Topics (Proceedings of the ICRA X Conference),45. AMS. Fields Institute Communications,pp.229-246.

[27] Mansfield E.L.,Szanto A. Elimination Theory for Differential Difference Polynomnials Proc. ISSAC 2002 (2002), 283-290, ACM

[28] F. Mora, De Nugis Groebnerialium 4: Zacharias, Spears, Möller Proc. ISSAC'15 (2015), 191-198, ACM

[29] T. Mora, Seven variations on standard bases, (1988)

ftp://ftp.disi.unige.it/person/MoraF/PUBLICATIONS/7Varietions.tar.gz

[30] T. Mora Solving Polynomial Equation Systems II: Macaulay's Paradigm and Gröbner Technology, Cambridge University Press (2005)

[31] T. Mora Zacharias Representeation of Effective Associative Rings, J. Symb. Comp. (submitted)

[32] Mosteig E., Sweedler M. Valuations and filtrations, J. Symb. Comp. 34 (2002), 399-435

[33] Möller H.M., New constructive methods in classical ideal theory, J. Algebra 100 (1986) $138-178$

[34] Möller H.M., On the construction of Gröbner bases using syzygies, J. Symb. Comp. 6 (1988), 345-359

[35] Norton G.H., Sălăgean A., Strong Gröbner bases for polynomials over a principal ideal ring, Bull. Austral. Math. Soc. 64 (2001), 505-528

[36] Norton G.H., Sălăgean A., Gröbner bases and products of coefficient rings, Bull. Austral. Math. Soc. 65 (2002), 147-154

[37] Ore O., Linear equations in non-commutative fields , Ann. Math. 32 (1931), 463477 
[38] Ore O., Theory of non-commutative polynomials , Ann. Math. 34 (1933), 480508.

[39] Pan L., On the D-bases of polynomial ideals over principal ideal domains, J. Symb. Comp. 7 (1988), 55-69

[40] Pesch M., Gröbner Bases in Skew Polynomial Rings Dissertation,Passau (1997)

[41] Pesch M., Two-sided Gröbner bases in Iterated Ore Extensions, Progress in Computer Science and Applied Logic 15 (1991), 225-243, Birkhäuser

[42] Pritchard F. L., A syzygies approach to non-commutative Gröbner bases, Preprint (1994)

[43] Pritchard F. L., The ideal membership problem in non-commutative polynomial rings, J. Symb. Comp. 22 (1996), 27-48

[44] Reinert B., A systematic Study of Gröbner Basis Methods, Habilitation, Kaiserslautern (2003)

[45] Reinert B., Gröbner Bases in Function Ring - A Guide for Introducing Reduction Relations to Algebraic Structures, J. Symb. Comp. 41 (2006), 1264-94

[46] Schreyer F.O., Die Berechnung von Syzygien mit dem verallgemeinerten Weierstrass'schen Divisionsatz, Diplomarbait, Hamburg (1980)

[47] Schreyer F.O., A standard basis approach to syzygies of canonical curves, J. Reine angew. Math. 421 (1991), 83-123

[48] Spear D.A., A constructive approach to commutative ring theory, in Proc. of the 1977 MACSYMA Users' Conference, NASA CP-2012 (1977), 369-376

[49] Sweedler M. Ideal bases and valuation rings, Manuscript (1986) available at http: //math.usask.ca/fvk/Valth.html

[50] Szekeres L., A canonical basis for the ideals of a polynomial domain, Am. Math. Monthly 59 (1952), 379-386

[51] Tamari D. On a certain Classification of rings and semigroups Bull. A.M.S. 54 (1948), 153-158

[52] Weispfenning, V. Finite Gröbner bases in non-noetherian Skew Polynomial Rings Proc. ISSAC'92 (1992), 320-332, A.C.M.

[53] Zacharias G., Generalized Gröbner bases in commutative polynomial rings, Bachelor's thesis, M.I.T. (1978) 